\title{
Highly Functionalized Pyranopyrans from Furans: A Synthesis of the C27-C38 and C44-C53 Subunits of Norhalichondrin B
}

\author{
James A. Henderson, Katrina L. Jackson, and Andrew J. Phillips* \\ Department of Chemistry and Biochemistry, University of Colorado, Boulder, CO 80309-0215
}

\section{SUPPORTING INFORMATION}

\begin{abstract}
General Experimental Procedures.
${ }^{1} \mathrm{H}$ and ${ }^{13} \mathrm{C}$ NMR spectra were recorded at $25^{\circ} \mathrm{C}$ on a Varian Inova spectrometer at 500 and $125 \mathrm{MHz}$, respectively, using $\mathrm{CDCl}_{3}$ as the solvent and internal reference. Coupling constants are reported in hertz, Hz. All non-aqueous reactions were run in flame-dried glassware under a dry $\mathrm{N}_{2}$ atmosphere. Toluene, THF, $\mathrm{CH}_{2} \mathrm{Cl}_{2}$, and $\mathrm{Et}_{2} \mathrm{O}$ were obtained from Aldrich (Pure-Pac) and further dried by passage through activated alumina as described by Bergman and Grubbs. ${ }^{1}$ All flash chromatography was performed with normal phase silica gel (Sorbent Technologies, 32-63 $\mu \mathrm{m}$ particle size, $60 \AA$ pore size), following the general protocol of Still. ${ }^{2}$
\end{abstract}

Synthesis of $(1 S, 2 S)-1-\{5$-[2-(tert-Butyl-dimethyl-silanyloxy)-ethyl]-furan-2-yl\}-2-methyl-but-3-en-1ol, 8:

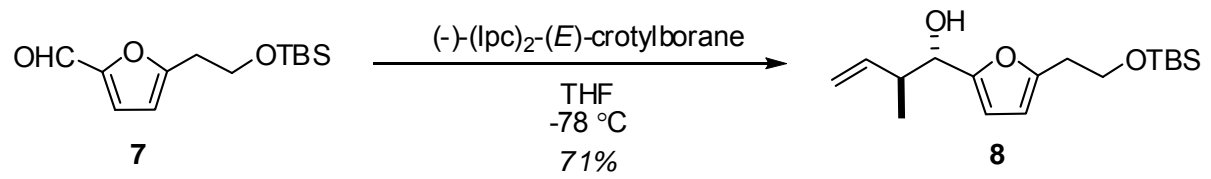

Freshly sublimed $\left(220^{\circ} \mathrm{C}, 1 \mathrm{~mm} \mathrm{Hg}\right)$ potassium tert-butoxide $(3.9 \mathrm{~g}, 18.42 \mathrm{mmol}, 1.2 \mathrm{eq})$ was dissolved in THF $(5.3 \mathrm{~mL})$ and the mixture was cooled to $-78^{\circ} \mathrm{C}$. Condensed trans-2-butene $(14.3 \mathrm{~mL}, 153.47 \mathrm{mmol}$, $10.0 \mathrm{eq})$ at $-78{ }^{\circ} \mathrm{C}$ was cannulated into the reaction mixture, then $n$-butyllithium $(1.6 \mathrm{M}$ in hexanes, 11.5 $\mathrm{mL}, 18.42 \mathrm{mmol}, 1.2 \mathrm{eq}$ ) was added dropwise. The resultant solution was warmed to $-45^{\circ} \mathrm{C}$ for 20 minutes, then re-cooled to $-78{ }^{\circ} \mathrm{C}$. (-)-(Ipc $)_{2} \mathrm{BOMe}\left(1 \mathrm{M}\right.$ in $\left.\mathrm{Et}_{2} \mathrm{O}, 20 \mathrm{~mL}, 19.95 \mathrm{mmol}, 1.3 \mathrm{eq}\right)$ was added dropwise, and the solution was stirred $40 \mathrm{~min}$. Boron trifluoride etherate $(2.7 \mathrm{~mL}, 21.49 \mathrm{mmol}, 1.4 \mathrm{eq})$ was then added dropwise, followed by aldehyde $7(3.9 \mathrm{~g}, 15.35 \mathrm{mmol}, 1.0 \mathrm{eq})$ dropwise. The reaction was stirred $3 \mathrm{~h}$ at $-78{ }^{\circ} \mathrm{C}$, then quenched by the addition of $3 \mathrm{M} \mathrm{NaOH}(8.7 \mathrm{~mL})$ and $30 \%$ hydrogen peroxide ( 3.9 $\mathrm{mL}$ ). The resultant mixture was stirred at ambient temperature for 12 hours. The layers were separated and the aqueous was extracted with EtOAc. The combined organic phase was washed with $\mathrm{H}_{2} \mathrm{O}$ and brine, dried over $\mathrm{MgSO}_{4}$, filtered, and concentrated. The crude residue was purified by flash column chromatography (5\% EtOAc/Hexanes) to yield $\mathbf{8}(3.38 \mathrm{~g}, 71 \%$ yield $)$ as a clear oil.

Data for 8: $[\boldsymbol{\alpha}]_{\mathbf{D}}-47.70\left(c=1.0, \mathrm{CHCl}_{3}\right) ;{ }^{1} \mathbf{H} \mathbf{N M R}\left(500 \mathrm{MHz}, \mathrm{CDCl}_{3}\right) \delta 6.16(\mathrm{~d}, 1 \mathrm{H}, \mathrm{J}=3.2), 6.00(\mathrm{~d}, 1 \mathrm{H}$, $\mathrm{J}=3.2), 5.77-5.86(\mathrm{~m}, 1 \mathrm{H}), 5.15-5.24(\mathrm{~m}, 2 \mathrm{H}), 4.35(\mathrm{~d}, 1 \mathrm{H}, \mathrm{J}=7.8), 3.84(\mathrm{t}, 2 \mathrm{H}, \mathrm{J}=6.8), 2.84(\mathrm{t}, 2 \mathrm{H}, \mathrm{J}=$ 6.8), 2.65-2.74 (m, 1H), $2.1(\mathrm{bs}, 1 \mathrm{H}), 0.95(\mathrm{~d}, 3 \mathrm{H}, \mathrm{J}=6.8), 0.88(\mathrm{~s}, 9 \mathrm{H}), 0.03(\mathrm{~s}, 6 \mathrm{H}) ;{ }^{13} \mathbf{C}$ NMR $(100 \mathrm{MHz}$, $\left.\mathrm{CDCl}_{3}\right) \delta 153.22,152.91,140.18,116.81,108.03,106.64,71.38,61.57,43.43,31.94,25.84,18.25,16.39$, 5.43; IR (neat, $\mathrm{cm}^{-1}$ ) 3434.6, 2929.1, 1558.7, 1471.9, 1103.2, 836.5; HRMS $\mathrm{m} / \mathrm{z}$ (ESI) calc'd for $\mathrm{C}_{17} \mathrm{H}_{30} \mathrm{O}_{3} \mathrm{SiNa}^{+}\left(\mathrm{M}^{+}+\mathrm{Na}\right) 333.1856$, found 333.1858 .

\footnotetext{
${ }^{1}$ a) Alaimo, P. J.; Peters, D.W.; Arnold, J.; Bergman, R.G. J. Chem. Educ. 200178 64. b) Pangborn, A. B.; Giardello, M. A.; Grubbs, R. H.; Rosen, R.K.; Timmers, F. J. Organometallics 1996, 15, 151.

${ }^{2}$ Still, W.C.; Kahn, M.; Mitra, A. J. Org. Chem. 1978, 43, 2923.
} 


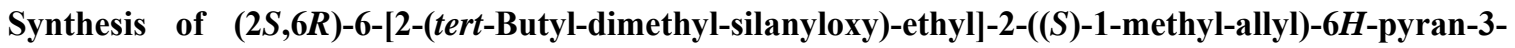
one, 10:

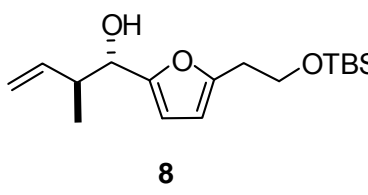

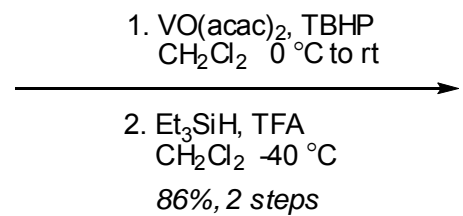

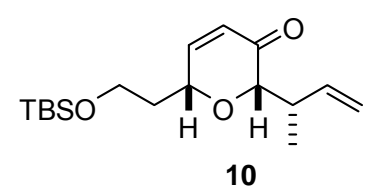

10

To a stirred solution of furyl alcohol $8(3.2 \mathrm{~g}, 10.31 \mathrm{mmol}, 1.0 \mathrm{eq})$ in $\mathrm{CH}_{2} \mathrm{Cl}_{2}(52 \mathrm{~mL})$ at $0{ }^{\circ} \mathrm{C}$ was added vanadyl acetylacetonate $(273 \mathrm{mg}, 1.03 \mathrm{mmol}, 0.1 \mathrm{eq})$ followed by dropwise tert-butylhydroperoxide (3.5 $\mathrm{mL}, 17.52 \mathrm{mmol}, 1.7 \mathrm{eq})$. The solution was stirred 40 minutes at $0{ }^{\circ} \mathrm{C}$, then $1 \mathrm{hr}$ at room temperature. Saturated aqueous sodium sulfite $(50 \mathrm{~mL})$ was added, and stirring was continued for 20 minutes. The resultant biphasic mixture was poured into EtOAc. The layers were separated and the aqueous extracted with EtOAc. The combined organic phase was washed with $\mathrm{H}_{2} \mathrm{O}$ and brine, dried over $\mathrm{MgSO}_{4}$, filtered, and concentrated. The crude residue was taken directly on the following reaction.

The crude residue from the previous reaction was dissolved in $\mathrm{CH}_{2} \mathrm{Cl}_{2}(108 \mathrm{~mL})$ and cooled to $-40{ }^{\circ} \mathrm{C}$. Triethylsilane $(8.2 \mathrm{~mL}, 51.53 \mathrm{mmol}, 5 \mathrm{eq})$ was added dropwise, followed by dropwise addition of trifluoroacetic acid $(12.0 \mathrm{~mL})$. The resultant solution was stirred at $-40{ }^{\circ} \mathrm{C}$ for $1.5 \mathrm{hrs}$. The reaction mixture was carefully poured into cold saturated aqueous $\mathrm{NaHCO}_{3}$. The layers were separated, and the aqueous extracted with EtOAc. The combined organic phase was washed with saturated aqueous $\mathrm{NaHCO}_{3}$ and brine, dried over $\mathrm{MgSO}_{4}$, filtered, and concentrated. The crude residue was purified by flash column chromatography (10\% EtOAc/Hexanes) to yield $\mathbf{1 0}$ as a pale yellow oil (2.75 g, $86 \%$ yield for 2 steps).

Data for 10: $[\boldsymbol{\alpha}]_{\mathbf{D}}+10.86\left(c=1.0, \mathrm{CHCl}_{3}\right) ;{ }^{1} \mathbf{H}$ NMR $\left(500 \mathrm{MHz}, \mathrm{CDCl}_{3}\right) \delta 6.97(\mathrm{dd}, 1 \mathrm{H}, \mathrm{J}=1.4,10.3)$, $6.07(\mathrm{dd}, 1 \mathrm{H}, \mathrm{J}=2.4,10.3), 5.78-5.87(\mathrm{~m}, 1 \mathrm{H}), 4.97-5.09(\mathrm{~m}, 2 \mathrm{H}), 4.44-4.50(\mathrm{~m}, 1 \mathrm{H}), 3.89(\mathrm{t}, 1 \mathrm{H}, \mathrm{J}=2.4)$, 3.77-3.90 (m, 2H), 2.98-3.06 (m, 1H), 1.81-1.95 (m, 2H), $1.17(\mathrm{~d}, 3 \mathrm{H}, \mathrm{J}=7.0), 0.91(\mathrm{~s}, 9 \mathrm{H}), 0.07(\mathrm{~d}, 6 \mathrm{H}, \mathrm{J}$ $=3.7) ;{ }^{13}$ C NMR $\left(100 \mathrm{MHz}, \mathrm{CDCl}_{3}\right) \delta 196.09,152.04,138.66,127.16,115.42,83.95,70.56,58.60,38.08$, 37.67, 25.87, 18.26, 16.83, -5.40, -5.49; IR (neat, $\mathrm{cm}^{-1}$ ) 3078.3, 2955.7, 2928.9, 1692.9, 1092.1; HRMS (ESI) $\mathrm{m} / \mathrm{z}$ calc'd for $\mathrm{C}_{17} \mathrm{H}_{30} \mathrm{O}_{3} \mathrm{SiNa}^{+}\left(\mathrm{M}^{+}+\mathrm{Na}\right) 333.1856$, found 333.1860 .

\section{Synthesis of (3aR,5S,7aR)-5-((S)-but-3-en-2-yl)-tetrahydro-5H-furo[3,2-b]pyran-2,6-dione XX:}

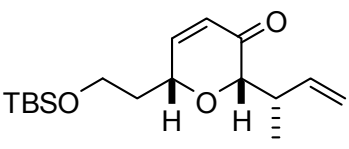

10

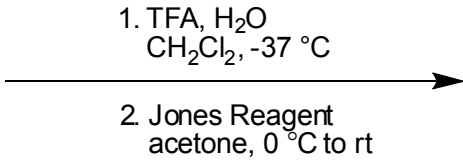

63\%, 2 steps

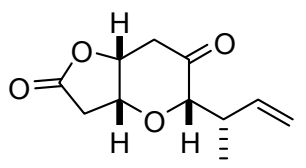

11

Silyl ether 10 (2.5 g, $8.05 \mathrm{mmol}, 1 \mathrm{eq})$ was dissolved in $\mathrm{CH}_{2} \mathrm{Cl}_{2}(73 \mathrm{~mL})$. Water $(2.1 \mathrm{~mL}, 120.77 \mathrm{mmol}, 15$ eq) was added, and the mixture was cooled to $-37^{\circ} \mathrm{C}$ with vigorous stirring. Trifluoroacetic acid $(8.0 \mathrm{~mL})$ was added in dropwise fashion so as to maintain the reaction temperature at $-37{ }^{\circ} \mathrm{C}$ (warming of the reaction mixture results in conjugate addition of the resultant alcohol into the enone). The reaction was followed closely by TLC and quenched immediately upon disappearance of starting material ( $4 \mathrm{hrs} \mathrm{total}$ reaction time). The mixture was carefully poured into ice-cold saturated aqueous $\mathrm{NaHCO}_{3}$ and stirred for 30 minutes. The layers were separated, and the aqueous extracted with EtOAc. The combined organics were washed with saturated aqueous $\mathrm{NaHCO}_{3}, \mathrm{H}_{2} \mathrm{O}$, and brine, and dried over $\mathrm{MgSO}_{4}$. The organics were then filtered and concentrated, and the crude was taken directly on to the following reaction.

The crude deprotected alcohol was dissolved in acetone $(67 \mathrm{~mL})$ and cooled to $0{ }^{\circ} \mathrm{C}$. Freshly prepared $1 \mathrm{M}$ aqueous Jones reagent $(20 \mathrm{~mL}, 20.13 \mathrm{mmol})$ was added dropwise, and the solution was stirred $1.5 \mathrm{hrs}$ at 0 ${ }^{\circ} \mathrm{C}$ and 30 minutes at room temperature. The reaction was quenched by addition of 2-propanol $(50 \mathrm{~mL})$. The blue mixture was filtered over Celite $^{\circledR}$ and the filtrate concentrated by rotary evaporation (bath 
temperature at $50{ }^{\circ} \mathrm{C}$ ). The resultant residue was then taken up in $\mathrm{CH}_{2} \mathrm{Cl}_{2}$, filtered again over Celite ${ }^{\circledR}$, and concentrated to a yellow oil. The crude residue was purified by flash column chromatography $(30 \%$ EtOAc/Hexanes) to yield $\mathbf{1 1}$ (1.05 g, 63\% for 2 steps) as a pale yellow oil.

Data for 11: $[\boldsymbol{\alpha}]_{\mathbf{D}}-151.1\left(c=1.0, \mathrm{CHCl}_{3}\right) ;{ }^{1} \mathbf{H}$ NMR $\left(500 \mathrm{MHz}, \mathrm{CDCl}_{3}\right) \delta 5.71-5.80(\mathrm{~m}, 1 \mathrm{H}), 4.97-5.05(\mathrm{~m}$, $2 \mathrm{H}), 4.77(\mathrm{dt}, 1 \mathrm{H}, \mathrm{J}=4.5,7.3), 4.24-4.28(\mathrm{~m}, 1 \mathrm{H}), 3.72(\mathrm{~d}, 1 \mathrm{H}, \mathrm{J}=3.1), 3.03(\mathrm{dd}, 1 \mathrm{H}, \mathrm{J}=7.8,14.9), 2.95$ $(\mathrm{dd}, 1 \mathrm{H}, \mathrm{J}=7.0,18.6), 2.68-2.81(\mathrm{~m}, 3 \mathrm{H}), 1.16(\mathrm{~d}, 3 \mathrm{H}, \mathrm{J}=7.0) ;{ }^{13} \mathbf{C}$ NMR $\left(100 \mathrm{MHz}, \mathrm{CDCl}_{3}\right) \delta 208.79$, 174.06, 137.01, 116.92, 86.98, 77.67, 73.18, 42.80, 40.85, 35.50, 17.03; IR (neat, $\mathrm{cm}^{-1}$ ) 3082.7, 2976.7, 1786.2, 1729.1, 1166.5, 1097.17; HRMS $m / z$ (ESI) calc'd for $\mathrm{C}_{11} \mathrm{H}_{14} \mathrm{O}_{4} \mathrm{Na}^{+}\left(\mathrm{M}^{+}+\mathrm{Na}\right)$ 233.0784, found 233.0787 .

Synthesis of (3aR,5S,6S,7aR)-5-((S)-but-3-en-2-yl)-hexahydro-6-hydroxyfuro[3,2-b]pyran-2-one, 12:

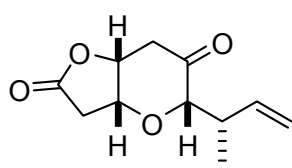

11

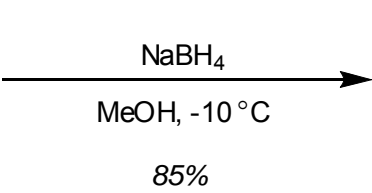

$85 \%$

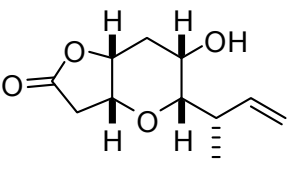

12

Ketone 11 (100 mg, $0.48 \mathrm{mmol}, 1 \mathrm{eq})$ was dissolved in $\mathrm{MeOH}(4.8 \mathrm{~mL})$ and cooled to $-10{ }^{\circ} \mathrm{C}$. Sodium borohydride ( $27 \mathrm{mg}, 0.71 \mathrm{mmol}, 1.5 \mathrm{eq})$ was added over 5 minutes, and the reaction was allowed to stir a further 20 minutes at $-10{ }^{\circ} \mathrm{C}$. The reaction was quenched by pouring over saturated aqueous $\mathrm{NH}_{4} \mathrm{Cl}$. The layers were separated and the aqueous extracted with EtOAc. The combined organics were washed with brine, dried $\left(\mathrm{MgSO}_{4}\right)$, filtered, and concentrated. The crude residue was purified by flash column chromatography (50\% EtOAc/Hexanes) yield alcohol 12 (86 mg, 85\% yield) as a pale yellow oil.

Data for 12: $[\boldsymbol{\alpha}]_{\mathbf{D}}-19.19\left(c=1.0, \mathrm{CHCl}_{3}\right) ;{ }^{1} \mathbf{H}$ NMR $\left(500 \mathrm{MHz}, \mathrm{CDCl}_{3}\right) \delta 5.82-5.92(\mathrm{~m}, 1 \mathrm{H}), 4.96-5.05(\mathrm{~m}$, $2 \mathrm{H}), 4.36-4.40(\mathrm{~m}, 1 \mathrm{H}), 4.22-4.26(\mathrm{~m}, 1 \mathrm{H}), 3.79(\mathrm{t}, 1 \mathrm{H}, \mathrm{J}=2.9), 3.03(\mathrm{~d}, 1 \mathrm{H}, \mathrm{J}=9.3), 2.70(\mathrm{dd}, 1 \mathrm{H}, \mathrm{J}=$ 17.4, 4.2), 2.54-2.64 (m, 2H), 2.42-2.51 (m, 1H), 2.18 (bs, 1H), 1.93 (dt, $1 \mathrm{H}, \mathrm{J}=16.0,4.2), 0.98$ (d, 3H, J = 6.9); ${ }^{13} \mathbf{C}$ NMR $\left(100 \mathrm{MHz}, \mathrm{CDCl}_{3}\right) \delta 175.38,141.06,114.15,81.57,76.17,74.06,62.49,38.36,37.78$, 32.11, 15.03; IR (neat, $\mathrm{cm}^{-1}$ ) 3482.2, 2927.8, 1769.9, 1631.3, 1166.5, 1101.3; HRMS (ESI) $\mathrm{m} / \mathrm{z}$ calc'd for $\mathrm{C}_{11} \mathrm{H}_{16} \mathrm{O}_{4} \mathrm{Na}^{+}\left(\mathrm{M}^{+}+\mathrm{Na}\right)$ 235.0941, found 235.0942.

Synthesis of [(2R,3R,5S,6S)-3,5-Bis-(tert-butyl-dimethyl-silanyloxy)-6-((S)-1-methyl-allyl)-tetrahydropyran-2-yl]-acetic acid methyl ester, 13:
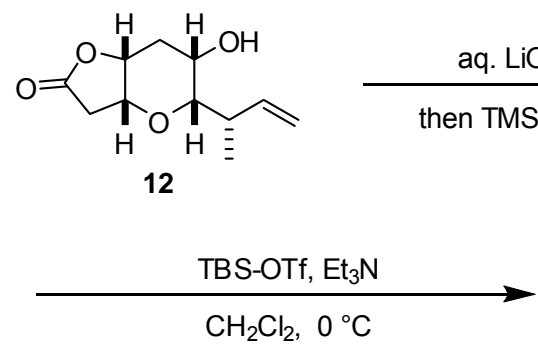
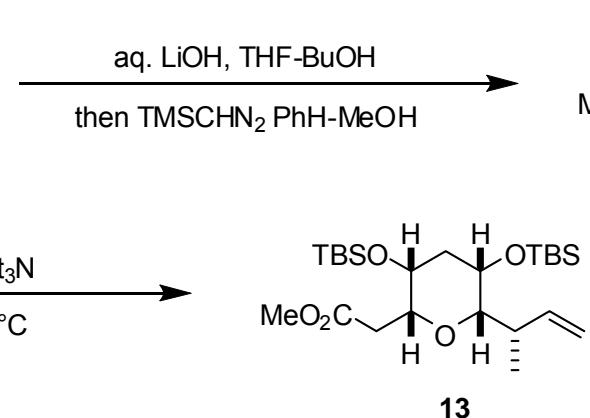

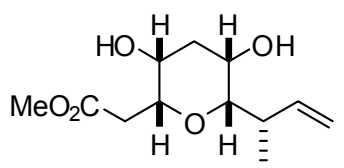

(78\%, 2 steps)

a) Lactone $12(0.36 \mathrm{~g}, 1.7 \mathrm{mmol}, 1.0 \mathrm{eq})$ was dissolved in $5: 4$ THF- $t$-BuOH $(175 \mathrm{~mL})$. $1 \mathrm{M}$ aqueous LiOH $(96 \mathrm{~mL}, 55.0 \mathrm{eq})$ was added, and the reaction was stirred 3.5 hours at room temperature. The mixture was diluted with $\mathrm{Et}_{2} \mathrm{O}$ and acidified to $\mathrm{pH} 3$ with $1 \mathrm{M} \mathrm{HCl}$. The layers were separated and the aqueous exhaustively extracted with $\mathrm{Et}_{2} \mathrm{O}$. The combined organics were dried $\left(\mathrm{Na}_{2} \mathrm{SO}_{4}\right)$, filtered, and concentrated (at no higher than $23{ }^{\circ} \mathrm{C}$ rotovap bath temperature to prevent relactonization). The crude residue was dissolved in 7:2 PhH-MeOH (54 mL). Trimethylsilyldiazomethane ( $10 \%$ in decane, $3 \mathrm{~mL}, 2.62 \mathrm{mmol}, 1.5$ eq) was added dropwise at room temperature.

After 15 minutes reaction time, the solution was 
concentrated (rotovap bath temperature at or below $23{ }^{\circ} \mathrm{C}$ ) and purified by flash column chromatography in a solvent gradient $\left(50 \% \mathrm{Et}_{2} \mathrm{O} / \mathrm{Hexanes} \rightarrow 100 \% \mathrm{Et}_{2} \mathrm{O}\right)$ to yield the methyl ester as a pale yellow oil $(0.36 \mathrm{~g}$, $86 \%$ yield).

$[\boldsymbol{\alpha}]_{\mathbf{D}}-5.63\left(c=1.0, \mathrm{CHCl}_{3}\right) ;{ }^{1} \mathbf{H}$ NMR $\left(500 \mathrm{MHz}, \mathrm{CDCl}_{3}\right) \delta 5.92-6.00(\mathrm{~m}, 1 \mathrm{H}), 4.99-5.11(\mathrm{~m}, 2 \mathrm{H}), 3.92-$ $3.95(\mathrm{~m}, 1 \mathrm{H}), 3.79-3.84(\mathrm{~m}, 1 \mathrm{H}), 3.70-3.74(\mathrm{~m}, 1 \mathrm{H}), 3.68(\mathrm{~s}, 3 \mathrm{H}), 3.55(\mathrm{bd}, 1 \mathrm{H}, \mathrm{J}=8.0), 3.11(\mathrm{dd}, 1 \mathrm{H}, \mathrm{J}=$ $0.8,8.1), 3.09$ (bd, $1 \mathrm{H}, \mathrm{J}=5.4), 2.77$ (dd, $1 \mathrm{H}, \mathrm{J}=8.1,15.5), 2.60(\mathrm{dd}, 1 \mathrm{H}, \mathrm{J}=5.6,15.5), 2.47-2.50(\mathrm{~m}, 1 \mathrm{H})$, $2.27(\mathrm{dt}, 1 \mathrm{H}, \mathrm{J}=3.0,14.8), 1.79(\mathrm{dt}, 1 \mathrm{H}, \mathrm{J}=3.1,14.8) ;{ }^{13} \mathbf{C} \mathbf{N M R}\left(100 \mathrm{MHz}, \mathrm{CDCl}_{3}\right) \delta 172.16,141.65$, 114.20, 83.84, 77.25, 66.51, 65.85, 51.68, 38.83, 36.87, 36.58, 16.04; IR (neat, $\mathrm{cm}^{-1}$ ) 3400.7, 2931.8, 1737.3, 1439.6, 1084.9; HRMS (ESI) $\mathrm{m} / z$ calc'd for $\mathrm{C}_{12} \mathrm{H}_{20} \mathrm{O}_{5} \mathrm{Na}^{+}\left(\mathrm{M}^{+}+\mathrm{Na}\right)$ 267.1203, found 267.1196.

b) The methyl ester obtained above $(0.35 \mathrm{~g}, 1.43 \mathrm{mmol}, 1.0 \mathrm{eq})$, was was dissolved in $\mathrm{CH}_{2} \mathrm{Cl}_{2}(14.3 \mathrm{~mL})$ and cooled to $0{ }^{\circ} \mathrm{C}$. Triethylamine $(1.2 \mathrm{~mL}, 8.60 \mathrm{mmol}, 6.0 \mathrm{eq})$ was added over 5 minutes, followed by dropwise addition of tert-butyldimethylsilyl triflate $(0.99 \mathrm{~mL}, 4.30 \mathrm{mmol}, 3.0 \mathrm{eq})$. The reaction was stirred for 1 hour at $0{ }^{\circ} \mathrm{C}$ and then a second portion of $1.2 \mathrm{~mL}$ triethylamine and $0.99 \mathrm{~mL}$ tert-butyldimethylsilyl trfilate were added sequentially and dropwise. The reaction was stirred a further hour at $0{ }^{\circ} \mathrm{C}$ and then quenched by addition of saturated aqueous $\mathrm{NH}_{4} \mathrm{Cl}$. The layers were separated and the aqueous extracted with EtOAc. The combined organics were dried $\left(\mathrm{MgSO}_{4}\right)$, filtered, and concentrated. The crude residue was purified by flash column chromatography (5\% EtOAc/Hexanes) to yield $\mathbf{1 3}(0.61 \mathrm{~g}, 91 \%$ yield) as a pale yellow oil.

Data for 13: $[\boldsymbol{\alpha}]_{\mathbf{D}}-33.09\left(c=1.0, \mathrm{CHCl}_{3}\right) ;{ }^{1} \mathbf{H}$ NMR $\left(500 \mathrm{MHz}, \mathrm{CDCl}_{3}\right) \delta 5.91-5.99(\mathrm{~m}, 1 \mathrm{H}), 4.93-5.03(\mathrm{~m}$, $2 \mathrm{H}), 3.83-3.86(\mathrm{~m}, 1 \mathrm{H}), 3.79-3.83(\mathrm{~m}, 1 \mathrm{H}), 3.71-3.75(\mathrm{~m}, 1 \mathrm{H}), 3.67(\mathrm{~s}, 3 \mathrm{H}), 2.93(\mathrm{dd}, 1 \mathrm{H}, \mathrm{J}=1.8,9.6), 2.73$ $(\mathrm{dd}, 1 \mathrm{H}, \mathrm{J}=8.7,15.7), 2.58-2.67(\mathrm{~m}, 1 \mathrm{H}), 2.44(\mathrm{dd}, 1 \mathrm{H}, \mathrm{J}=4.6,15.7), 2.03(\mathrm{dt}, 1 \mathrm{H}, \mathrm{J}=2.9,14.9), 1.88$ (dt, $1 \mathrm{H}, \mathrm{J}=4.6,14.9), 0.92$ (s, 9H), 0.91 (s, 9H), $-0.2-0.13(\mathrm{~m}, 12 \mathrm{H}) ;{ }^{13} \mathbf{C} \mathbf{~ N M R}\left(100 \mathrm{MHz}, \mathrm{CDCl}_{3}\right) \delta 172.60$, $142.21,112.87,85.08,77.87,65.58,64.23,51.51,38.66,36.95,36.68,26.29,26.19,18.31,15.68,-2.56$, 3.75, -5.08, -5.16; IR (neat, $\mathrm{cm}^{-1}$ ) 2956.3, 1741.3, 1639.4, 1248.0, 1076.8; HRMS (ESI) $\mathrm{m} / \mathrm{z}$ calc'd for $\mathrm{C}_{24} \mathrm{H}_{48} \mathrm{O}_{5} \mathrm{Si}_{2} \mathrm{Na}^{+}\left(\mathrm{M}^{+}+\mathrm{Na}\right) 495.2932$, found 495.2919 .

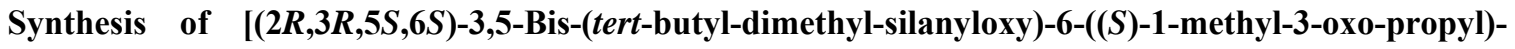
tetrahydro-pyran-2-yl]-acetic acid methyl ester, 5:

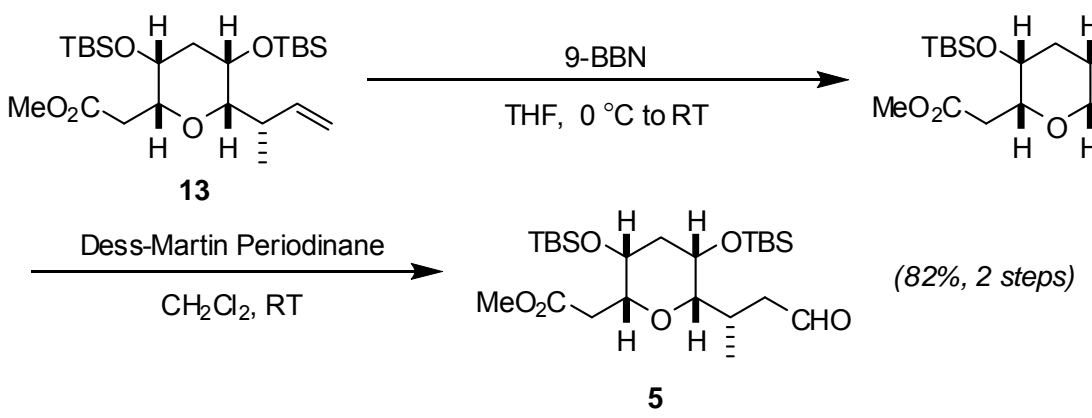

a) To a stirred solution of alkene $\mathbf{1 3}(2.06 \mathrm{~g}, 4.36 \mathrm{mmol}, 1 \mathrm{eq})$ in THF $(44 \mathrm{~mL})$ at $0{ }^{\circ} \mathrm{C}$ was added 9borabicyclo[3.3.1]nonane $(0.5 \mathrm{M}$ in THF, $17.4 \mathrm{~mL}, 8.72 \mathrm{mmol}, 2.0 \mathrm{eq})$ dropwise. The reaction solution was stirred a further 30 minutes at $0{ }^{\circ} \mathrm{C}$ and 2 hours at room temperature. After cooling back to $0{ }^{\circ} \mathrm{C}$, the reaction was quenched by addition of $3 \mathrm{M} \mathrm{NaOH}(4.4 \mathrm{~mL})$ and $30 \% \mathrm{H}_{2} \mathrm{O}_{2}(4.4 \mathrm{~mL})$. The biphasic mixture was warmed to room temperature and stirred for 12 hours. The layers were separated and the aqueous extracted with EtOAc. The combined organic phase was washed with brine, dried $\left(\mathrm{MgSO}_{4}\right)$, filtered, and concentrated. The crude residue was purified by flash column chromatography ( $30 \%$ EtOAc/hexanes) to yield the expected alcohol ( $1.78 \mathrm{~g}, 83 \%$ yield) as a pale yellow oil.

$[\boldsymbol{\alpha}]_{\mathbf{D}}+12.81\left(c=1.0, \mathrm{CHCl}_{3}\right) ;{ }^{\mathbf{1}} \mathbf{H}$ NMR $\left(500 \mathrm{MHz}, \mathrm{CDCl}_{3}\right) \delta 3.81-3.86(\mathrm{~m}, 2 \mathrm{H}), 3.64-3.73(\mathrm{~m}, 5 \mathrm{H}), 3.09$ $(\mathrm{dd}, 1 \mathrm{H}, \mathrm{J}=1.6,9.9), 2.80$ (dd, $1 \mathrm{H}, \mathrm{J}=9.5,16.4), 2.40(\mathrm{dd}, 1 \mathrm{H}, \mathrm{J}=3.6,16.4), 2.21$ (bs, 1H), 2.05-2.15 (m, $1 \mathrm{H}), 2.02(\mathrm{dt}, 1 \mathrm{H}, \mathrm{J}=2.6,15.0), 1.88(\mathrm{dt}, 1 \mathrm{H}, \mathrm{J}=4.6,15.0), 1.62-1.75(\mathrm{~m}, 2 \mathrm{H}), 0.93(\mathrm{~s}, 9 \mathrm{H}), 0.91(\mathrm{~s}, 9 \mathrm{H})$, 
$0.87(\mathrm{~d}, 3 \mathrm{H}, \mathrm{J}=6.9), 0.0-0.12(\mathrm{~m}, 12 \mathrm{H}) ;{ }^{13} \mathrm{C}$ NMR $\left(100 \mathrm{MHz}, \mathrm{CDCl}_{3}\right) \delta 172.93,84.97,77.40,65.68$, $64.12,60.75,51.72,38.76,36.81,35.53,30.22,26.30,26.20,18.31,16.01,-2.61,-3.70,-5.06,-5.11$; IR (neat, $\mathrm{cm}^{-1}$ ) $3470.0,2931.8,2854.4,1737.3,1146.1$; HRMS (ESI) $\mathrm{m} / z$ calc'd for $\mathrm{C}_{24} \mathrm{H}_{50} \mathrm{O}_{6} \mathrm{Si}_{2} \mathrm{Na}^{+}\left(\mathrm{M}^{+}+\mathrm{Na}\right)$ 513.3038, found 513.3024.

b) To a stirred solution of primary alcohol obtained above $(0.033 \mathrm{~g}, 0.067 \mathrm{mmol}, 1.0 \mathrm{eq})$ in $\mathrm{CH}_{2} \mathrm{Cl}_{2}(1.3$ $\mathrm{mL}$ ) was added Dess-Martin periodinane $(0.033 \mathrm{~g}, 0.067 \mathrm{mmol}, 1.0 \mathrm{eq})$. After stirring for $1 \mathrm{hr}$, a second portion of Dess-Martin periodinane $(0.033 \mathrm{~g}, 0.067 \mathrm{mmol}, 1.0 \mathrm{eq})$ was added and stirring was continued a further $1.5 \mathrm{hrs}$. The reaction was quenched by addition of $25 \mathrm{wt} \% \mathrm{Na}_{2} \mathrm{~S}_{2} \mathrm{O}_{3}$-doped saturated aqueous $\mathrm{NaHCO}_{3}$. The biphasic mixture was stirred until both layers became clear, then the phases were separated and the aqueous extracted with EtOAc. The combined organic phase was washed with brine, dried $\left(\mathrm{MgSO}_{4}\right)$, filtered, and concentrated. The residue was purifed by flash column chromatography in a solvent gradient (5\% EtOAc/Hexanes $\rightarrow 15 \%$ EtOAc/Hexanes) to yield 5 (.031 g, 95\% yield) as a white solid.

Data for 5: $[\boldsymbol{\alpha}]_{\mathbf{D}}+2.59\left(c=0.5, \mathrm{CHCl}_{3}\right) ;{ }^{1} \mathbf{H}$ NMR $\left(500 \mathrm{MHz}, \mathrm{CDCl}_{3}\right) \delta 9.65(\mathrm{t}, 1 \mathrm{H}, \mathrm{J}=2.3), 3.83-3.87(\mathrm{~m}$, $1 \mathrm{H}), 3.78-3.83(\mathrm{~m}, 1 \mathrm{H}), 3.70-3.73(\mathrm{~m}, 1 \mathrm{H}), 3.65-3.69(\mathrm{~m}, 4 \mathrm{H}), 2.95(\mathrm{dd}, 1 \mathrm{H}, \mathrm{J}=1.8,9.1), 2.67(\mathrm{dd}, 1 \mathrm{H}, \mathrm{J}=$ 8.7, 16.2), 2.50-2.61 (m, 2H), $2.41(\mathrm{dd}, 1 \mathrm{H}, \mathrm{J}=4.3,16.2), 2.16-2.24(\mathrm{~m}, 1 \mathrm{H}), 2.02(\mathrm{dt}, 1 \mathrm{H}, \mathrm{J}=2.7,14.9)$, 1.89 (dt, 1H, J = 4.6, 14.9), 0.913 (s, 9H), $0.911(\mathrm{~s}, 9 \mathrm{H}), 0.89(\mathrm{~d}, 3 \mathrm{H}, \mathrm{J}=6.6),-0.01-0.12(\mathrm{~m}, 12 \mathrm{H}) ;{ }^{13} \mathbf{C}$ NMR $\left(100 \mathrm{MHz}, \mathrm{CDCl}_{3}\right) \delta 203.44,172.28,84.83,77.79,65.47,63.89,51.60,47.89,38.53,36.62,28.85$, 26.27, 26.18, 18.27, 18.26, 16.73, -2.71, -3.72, -5.13, -5.19; IR (neat, $\mathrm{cm}^{-1}$ ) 2953.9, 2925.3, 2825.3, 2716.9, 1732.1, 1258.1, 1086.5; HRMS (ESI) $\mathrm{m} / \mathrm{z}$ calc'd for $\mathrm{C}_{24} \mathrm{H}_{48} \mathrm{O}_{6} \mathrm{Si}_{2} \mathrm{Na}^{+}\left(\mathrm{M}^{+}+\mathrm{Na}\right)$ 511.2882, found 511.2873.

Synthesis of (1S,2R)-1-(5-(2-(tert-Butyldimethylsilyloxy)ethyl)furan-2-yl)-2-methylbut-3-en-1-ol, 14:
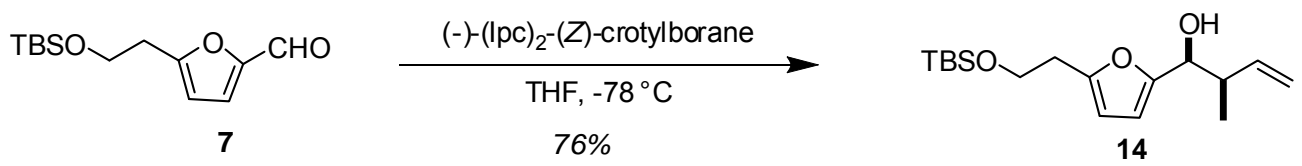

Freshly sublimed $\left(220^{\circ} \mathrm{C}, 1 \mathrm{~mm} \mathrm{Hg}\right)$ potassium tert-butoxide $(7.0 \mathrm{~g}, 61.38 \mathrm{mmol}, 1.2 \mathrm{eq})$ was dissolved in THF $(18 \mathrm{~mL})$, and the mixture was cooled to $-78{ }^{\circ} \mathrm{C}$. Condensed cis-2-butene $(23.7 \mathrm{~mL}, 255.77 \mathrm{mmol}, 5.0$ eq) was cannulated into the mixture, then $n$-butyllithium (1.6 M in hexanes, $38.3 \mathrm{~mL}, 61.38 \mathrm{mmol}, 1.2 \mathrm{eq}$.) was added dropwise. The resultant solution was warmed to $-50{ }^{\circ} \mathrm{C}$ for 20 minutes, then recooled to $-78^{\circ} \mathrm{C}$. $(-)-(\mathrm{Ipc})_{2} \mathrm{BOMe}\left(1 \mathrm{M}\right.$ in $\mathrm{Et}_{2} \mathrm{O}, 66.5 \mathrm{~mL}, 66.50 \mathrm{mmol}, 1.3 \mathrm{eq}$.) was added dropwise and the solution was stirred $30 \mathrm{~min}$. Boron trifluoride etherate $(9.0 \mathrm{~mL}, 71.61 \mathrm{mmol}, 1.4 \mathrm{eq})$ was added dropwise, followed by dropwise addition of aldehyde 7 (13.0 g, $51.15 \mathrm{mmol}, 1.0 \mathrm{eq}$.) in THF $(10 \mathrm{~mL})$. The reaction was stirred $3 \mathrm{~h}$ at $-78{ }^{\circ} \mathrm{C}$, then quenched by the addition of $3 \mathrm{M} \mathrm{NaOH}(13 \mathrm{~mL})$ and $30 \%$ hydrogen peroxide $(18 \mathrm{~mL})$. The resultant mixture was stirred at ambient temperature for 12 hours. The layers were separated and the aqueous layer was extracted with EtOAc. The combined organic phase was washed with $\mathrm{H}_{2} \mathrm{O}$ and brine, dried over $\mathrm{Na}_{2} \mathrm{SO}_{4}$, filtered and concentrated. The crude residue was purified by FCC $(5 \%$ EtOAc/Hexanes) to yield $\mathbf{1 4}(13.46 \mathrm{~g}, 76 \%$ yield $)$ as a clear oil.

Data for 14: $[\alpha]_{\mathrm{D}}-11.2\left(c 1.0, \mathrm{CHCl}_{3}\right){ }^{1} \mathbf{H}$ NMR $\left(500 \mathrm{MHz}, \mathrm{CDCl}_{3}\right) \delta 0.18(\mathrm{~s}, 6 \mathrm{H}), 0.87(\mathrm{~s}, 9 \mathrm{H}), 1.07(\mathrm{~d}, J$ $=7.0 \mathrm{~Hz}, 1 \mathrm{H}), 1.93(\mathrm{~d}, J=5.5 \mathrm{~Hz}, 1 \mathrm{H}), 2.66-2.70(\mathrm{~m}, 1 \mathrm{H}), 2.81(\mathrm{t}, J=7.0 \mathrm{~Hz}, 2 \mathrm{H}), 3.82(\mathrm{t}, J=7.0 \mathrm{~Hz}$, 2H), $4.49(\mathrm{t}, J=6.0 \mathrm{~Hz}, 1 \mathrm{H}), 5.04-5.11(\mathrm{~m}, 2 \mathrm{H}), 5.72-5.79(\mathrm{~m}, 1 \mathrm{H}), 5.98(\mathrm{~d}, J=3.5 \mathrm{~Hz}, 1 \mathrm{H}), 6.11(\mathrm{~d}, J=$ $3.0 \mathrm{~Hz}, 1 \mathrm{H}) ;{ }^{13} \mathbf{C}$ NMR $\left(100 \mathrm{MHz}, \mathrm{CDCl}_{3}\right) \delta-5.4,15.1,18.2,25.8,31.9,42.8,61.6,71.4,106.6,107.6$, 115.7, 139.7, 152.6, 153.5; IR (thin film) $3433(\mathrm{OH}), 2960,2923,2854,1472,1256,1109,999,905$, $836,779\left(\mathrm{~cm}^{-1}\right)$; HRMS (ESI) $\mathrm{m} / \mathrm{z}$ calc'd for $\mathrm{C}_{17} \mathrm{H}_{30} \mathrm{O}_{3} \mathrm{SiNa}\left(\mathrm{M}^{+}+\mathrm{Na}\right) 333.1856$, found 333.1847.

Syntheis of (2S,6R)-2-((R)-But-3-en-2-yl)-6-(2-(tert-butyldimethylsilyloxy)ethyl)-2H-pyran-3(6H)-one, 16: 


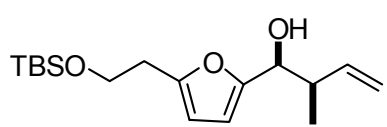

14

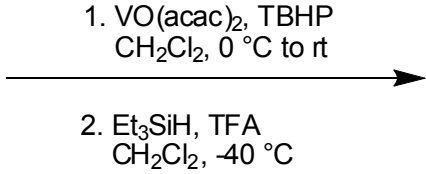

90\%, 2 steps

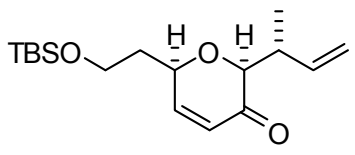

16

To a stirred solution of furyl alcohol $14(13.5 \mathrm{~g}, 43.47 \mathrm{mmol}, 1.0$ eq. $)$ in $\mathrm{CH}_{2} \mathrm{Cl}_{2}(220 \mathrm{~mL})$ at $0{ }^{\circ} \mathrm{C}$ was added vanadyl acetylacetonate $(1.15 \mathrm{~g}, 4.34 \mathrm{mmol}, 0.1 \mathrm{eq}$. $)$ followed by dropwise addition of tertbutylhydroperoxide $\left(13.4 \mathrm{~mL}, 73.91 \mathrm{mmol}, 1.7\right.$ eq.). The solution was stirred $40 \mathrm{~min}$ at $0{ }^{\circ} \mathrm{C}$, then $1 \mathrm{~h}$ at room temperature. Saturated aqueous sodium sulfite $(200 \mathrm{~mL})$ was added, and stirring was continued for 20 minutes. The resultant biphasic mixture was poured into EtOAc $(200 \mathrm{~mL})$. The layers were separated and the aqueous extracted with EtOAc. The combined organic phase was washed with $\mathrm{H}_{2} \mathrm{O}$ and brine, dried over $\mathrm{MgSO}_{4}$, filtered and concentrated. The crude residue was taken directly into the following reaction.

The crude hemiacetal from the previous reaction was dissolved in $\mathrm{CH}_{2} \mathrm{Cl}_{2}(400 \mathrm{~mL})$ and cooled to $-40{ }^{\circ} \mathrm{C}$. Triethylsilane $(34.7 \mathrm{~mL}, 217.39 \mathrm{mmol}, 5.0$ eq.) was added dropwise followed by the dropwise addition of trifluoroacetic acid $(43 \mathrm{~mL})$. The resultant solution was stirred at $-40^{\circ} \mathrm{C}$ for $1.5 \mathrm{hrs}$. The reaction mixture was then carefully poured into cold saturated aqueous $\mathrm{NaHCO}_{3}$. The layers were separated, and the aqueous layer was extracted with EtOAc. The combined organic phase was washed with saturated aqueous $\mathrm{NaHCO}_{3}$ and brine, dried over $\mathrm{Na}_{2} \mathrm{SO}_{4}$, filtered and concentrated. The crude residue was purified by $\mathrm{FCC}$ (10\% EtOAc/Hexanes) to yield $\mathbf{1 6}$ as a pale yellow oil (12.2 g, 90\% yield for 2 steps).

Data for 16: $[\alpha]_{\mathbf{D}}-14.2\left(c 1.0, \mathrm{CHCl}_{3}\right) ;{ }^{1} \mathbf{H}$ NMR $\left(500 \mathrm{MHz}, \mathrm{CDCl}_{3}\right) \delta 0.05(\mathrm{~d}, J=3.0 \mathrm{~Hz}, 6 \mathrm{H}), 0.89(\mathrm{~s}$, $9 \mathrm{H}), 0.99(\mathrm{~d}, J=7.0 \mathrm{~Hz}, 3 \mathrm{H}), 1.81-1.94(\mathrm{~m}, 2 \mathrm{H}), 2.98-3.04(\mathrm{~m}, 1 \mathrm{H}), 3.75-3.83(\mathrm{~m}, 2 \mathrm{H}), 3.96(\mathrm{t}, J=2.0 \mathrm{~Hz}$, $1 \mathrm{H}), 4.45-4.48(\mathrm{~m}, 1 \mathrm{H}), 5.01-5.12(\mathrm{~m}, 2 \mathrm{H}), 5.91-5.98(\mathrm{~m}, 1 \mathrm{H}), 6.08(\mathrm{dd}, J=2.5,10.0 \mathrm{~Hz}, 1 \mathrm{H}), 6.98(\mathrm{dd}, J$ $=1.5,10.0 \mathrm{~Hz}, 1 \mathrm{H}) ;{ }^{13} \mathbf{C}$ NMR $\left(100 \mathrm{MHz}, \mathrm{CDCl}_{3}\right) \delta-5.4,13.4,18.3,25.9,37.5,37.7,58.6,70.6,83.3$, 114.2, 127.1, 141.0, 152.3, 196.3; IR (thin film) 2956, 2927, 2882, 2858, 1745 (C=O), 1464, 1256, 1101, 844, $775\left(\mathrm{~cm}^{-1}\right)$; HRMS (EI) $\mathrm{m} / \mathrm{z}$ calc'd for $\mathrm{C}_{17} \mathrm{H}_{30} \mathrm{O}_{3} \mathrm{SiNa}\left(\mathrm{M}^{+}+\mathrm{Na}\right) 333.1856$, found 333.1855 .

\section{Synthesis of (3aR,5S,7aR)-5-((R)-but-3-en-2-yl)tetrahydro-2H-furo[3,2-b]pyran-2,6(5H)-dione XXX:}

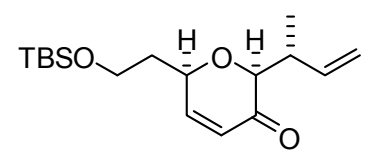

16

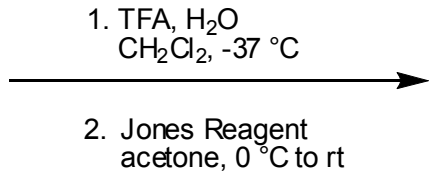

63\%, 2 steps

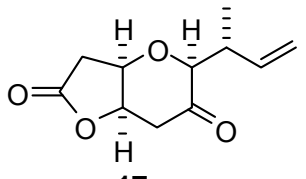

17

Protected alcohol 16 (13.0 g, $41.86 \mathrm{mmol})$ was dissolved in $\mathrm{CH}_{2} \mathrm{Cl}_{2}(376 \mathrm{~mL})$. Water $(23 \mathrm{~mL})$ was added and the mixture was cooled to $-37{ }^{\circ} \mathrm{C}$ with vigorous stirring. Trifluoroacetic acid $(41.8 \mathrm{~mL})$ was added slowly to maintain the reaction temperature at $-37^{\circ} \mathrm{C}$ (warming of the reaction mixture results in conjugate addition of the deprotected alcohol into the enone). The reaction was followed by TLC and quenched upon disappearance of starting material ( $\sim \mathrm{hrs}$ total reaction time). The mixture was carefully poured into icecold saturated aqueous $\mathrm{NaHCO}_{3}$ and stirred for 30 minutes. The layers were separated and the aqueous layer was extracted with EtOAc. The combined organics were washed with saturated aqueous $\mathrm{NaHCO}_{3}$, $\mathrm{H}_{2} \mathrm{O}$, brine, dried over $\mathrm{Na}_{2} \mathrm{SO}_{4}$, filtered and concentrated. The crude product was taken directly into the following reaction.

The crude deprotected alcohol was dissolved in acetone $(500 \mathrm{~mL})$ and cooled to $0{ }^{\circ} \mathrm{C}$. Freshly prepared $1 \mathrm{M}$ aqueous Jones reagent $(54 \mathrm{~mL}, 54 \mathrm{mmol})$ was added dropwise, and the solution was stirred $1.5 \mathrm{~h}$ at $0{ }^{\circ} \mathrm{C}$ and 30 minutes at room temperature. The reaction was quenched by addition of 2-propanol $(100 \mathrm{~mL})$. The resulting blue mixture was filtered over Celite ${ }^{\circledR}$ and the filtrate was concentrated by rotary evaporation (rotovap bath temperature $50^{\circ} \mathrm{C}$ ). The resultant residue was then taken up in $\mathrm{CH}_{2} \mathrm{Cl}_{2}$, filtered over Celite ${ }^{\circledR}$ 
and concentrated to a yellow oil. The crude product was purified by flash column chromatography ( $30 \%$ EtOAc/Hexanes) to yield 17 (5.54 g, 63\% 2 steps) as a pale yellow oil.

Data for 17: $[\alpha]_{\mathbf{D}}-106.7\left(c 1.0, \mathrm{CHCl}_{3}\right) ;{ }^{1} \mathbf{H}$ NMR $\left(500 \mathrm{MHz}, \mathrm{CDCl}_{3}\right) \delta 1.10(\mathrm{~d}, J=5.0 \mathrm{~Hz}, 3 \mathrm{H}), 2.72-2.81$ (m, 3H), 2.91-2.96 (m, 1H), 3.02-3.07 (m, 1H), $3.73(\mathrm{~d}, J=3.5 \mathrm{~Hz}, 1 \mathrm{H}), 4.27-4.29(\mathrm{~m}, 1 \mathrm{H}), 4.76-4.80(\mathrm{~m}$, $1 \mathrm{H}), 5.05-5.10(\mathrm{~m}, 2 \mathrm{H}), 5.80-5.87(\mathrm{~m}, 1 \mathrm{H}) ;{ }^{13} \mathbf{C} \mathbf{~ N M R}\left(100 \mathrm{MHz}, \mathrm{CDCl}_{3}\right) \delta$ 13.7, 35.5, 39.5, 42.6, 73.1, 77.5, 86.1 115.6, 138.8, 174.0, 208.2; IR (thin film) 2980, 2935, 1786, 1729, 1402, 1199, 1166, 1093, 1044, $917\left(\mathrm{~cm}^{-1}\right)$; HRMS (EI) $\mathrm{m} / \mathrm{z}$ calc'd for $\mathrm{C}_{11} \mathrm{H}_{14} \mathrm{O}_{4} \mathrm{Na}\left(\mathrm{M}^{+}+\mathrm{Na}\right) 233.0784$, found 233.0783.

\section{Synthesis of (3aR,5S,6S,7aR)-5-((R)-But-3-en-2-yl)-6-hydroxyhexahydro-2H-furo[3,2-b]pyran-2-one} 18:
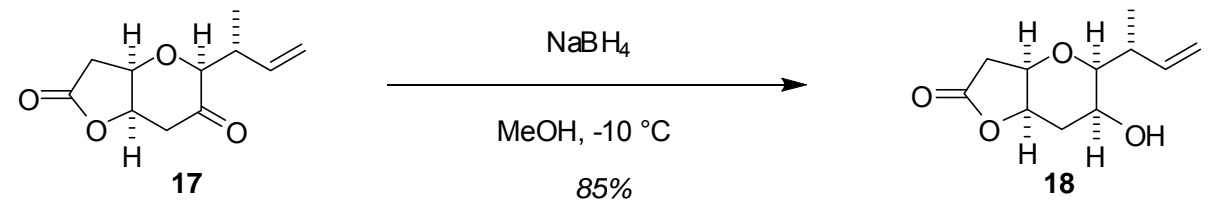

Ketone 17(4.8 g, $22.83 \mathrm{mmol}, 1$ eq.) was dissolved in $\mathrm{MeOH}$ and cooled to $-10{ }^{\circ} \mathrm{C}$. Sodium borohydride ( $950 \mathrm{mg}, 25.11 \mathrm{mmol}, 1.1$ eq.) was added over $5 \mathrm{~min}$, and the reaction was allowed to stir for an additional 20 minutes at $-10{ }^{\circ} \mathrm{C}$. The reaction was quenched by pouring into saturated aqueous $\mathrm{NH}_{4} \mathrm{Cl}$. The layers were separated and the aqueous phase was extracted with EtOAc. The combined organics were washed with brine, dried $\left(\mathrm{Na}_{2} \mathrm{SO}_{4}\right)$, filtered and concentrated. The crude residue was purified by flash column chromatography (50\% EtOAc/Hexanes) to yield alcohol $\mathbf{1 8}(4.11 \mathrm{~g}, 85 \%$ yield) as a clear oil.

Data for 18: $[\alpha]_{\mathbf{D}}+36.1\left(\mathrm{c} 1.0, \mathrm{CHCl}_{3}\right) ;{ }^{1} \mathbf{H}$ NMR $\left(500 \mathrm{MHz}, \mathrm{CDCl}_{3}\right) \delta 1.07(\mathrm{~d}, J=6.5 \mathrm{~Hz}, 3 \mathrm{H}), 1.91(\mathrm{dt}, J$ $=4.0,16.0 \mathrm{~Hz}, 1 \mathrm{H}), 2.52-2.72(\mathrm{~m}, 4 \mathrm{H}), 2.98(\mathrm{~d}, \mathrm{~J}=8.0 \mathrm{~Hz}, 1 \mathrm{H}), 3.78(\mathrm{br} \mathrm{s}, 1 \mathrm{H}), 4.28-4.29(\mathrm{~m}, 1 \mathrm{H}), 4.39-$ $4.40(\mathrm{~m}, 1 \mathrm{H}), 5.04-5.17(\mathrm{~m}, 2 \mathrm{H}), 5.61-5.68(\mathrm{~m}, 1 \mathrm{H}) ;{ }^{13} \mathbf{C}$ NMR $\left(100 \mathrm{MHz}, \mathrm{CDCl}_{3}\right) \delta 16.4,32.1,38.4,38.5$, 62.5, 74.2, 76.2, 81.5, 116.2, 138.9, 175.3; IR (thin film) 3498 (OH), 2960, 2932, 1778, 1407, 1296, 1199, 1154, 1093, 1036, 995, 913, $881\left(\mathrm{~cm}^{-1}\right)$; HRMS (EI) $\mathrm{m} / \mathrm{z}$ calc'd for $\mathrm{C}_{11} \mathrm{H}_{16} \mathrm{O}_{4} \mathrm{Na}\left(\mathrm{M}^{+}+\mathrm{Na}\right) 235.0940$, found 235.0936.

\section{Synthesis of $((2 S, 3 S, 5 R, 6 R)-2-((R)$-But-3-en-2-yl)-6-(2-(tert-butyldimethylsilyloxy)ethyl)tetrahydro- 2H-pyran-3,5-diyl)bis(oxy)bis(tert-butyldimethylsilane), 19:}

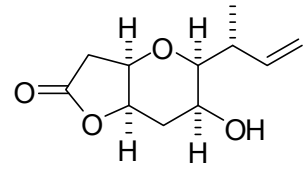

18

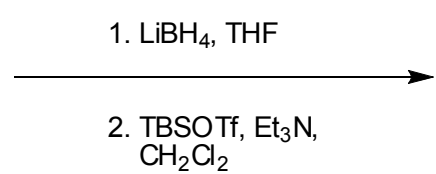

75\%, 2 steps

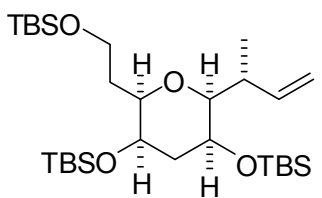

19

Lactone 18 (1.8 g, $8.48 \mathrm{mmol}, 1.0$ eq.) was dissolved in THF $(80 \mathrm{~mL})$ and cooled to $0{ }^{\circ} \mathrm{C}$. $\mathrm{LiBH}_{4}(17.0$ $\mathrm{mL}, 33.92 \mathrm{mmol}, 2.0 \mathrm{M}$ in THF, 4 eq.) was added slowly and the reaction mixture was warmed to room temperature. The reaction was heated to reflux for $1 \mathrm{~h}$ then recooled to room temperature. The reaction was quenched with $\mathrm{MeOH}(24 \mathrm{~mL})$ and then concentrated under reduced pressure. The residue was dissolved in $\mathrm{Et}_{2} \mathrm{O}(100 \mathrm{~mL})$, washed with $\mathrm{H}_{2} \mathrm{O}$ and brine, dried $\mathrm{Na}_{2} \mathrm{SO}_{4}$, filtered and concentrated. The crude product was taken directly into the following reaction.

The triol from above was dissolved in $\mathrm{CH}_{2} \mathrm{Cl}_{2}(60 \mathrm{~mL})$ and cooled to $0{ }^{\circ} \mathrm{C}$. To this solution was added triethylamine $(9.0 \mathrm{~mL}, 64.4 \mathrm{mmol})$ followed by t-butyldimethylsilytriflate $(7.4 \mathrm{~mL}, 32.4 \mathrm{mmol})$. After $1 \mathrm{~h}$, additional triethyl amine $(9.0 \mathrm{~mL}, 64.4 \mathrm{mmol})$ and $t$-butyldimethylsilyltriflate $(7.4 \mathrm{~mL}, 32.4 \mathrm{mmol})$ was added and the reaction was stirred for $2 \mathrm{~h}$. The reaction was quenched with saturated $\mathrm{NaHCO}_{3}$. The resulting mixture was extracted with EtOAc and the combined organics were washed with brine, dried 
$\mathrm{Na}_{2} \mathrm{SO}_{4}$, filtered and concentrated. The crude residue was purified by flash column chromatography ( $2 \%$ EtOAc/Hex) to provide $\mathbf{1 9}$ (3.55 g, 75\% over 2 steps) as a clear oil.

Data for 19: $[\alpha]_{\mathbf{D}}+25.6\left(\right.$ c $\left.1.0, \mathrm{CHCl}_{3}\right) ;{ }^{1} \mathbf{H}$ NMR $\left(500 \mathrm{MHz}, \mathrm{CDCl}_{3}\right) \delta 0.03(\mathrm{~s}, 12 \mathrm{H}), 0.08(\mathrm{~d}, J=22.5 \mathrm{~Hz}$, $6 \mathrm{H}), 0.88$ (s, 9H), 0.91 (s, 9H), $0.92(\mathrm{~s}, 9 \mathrm{H}), 1.10(\mathrm{~d}, J=6.5 \mathrm{~Hz}, 3 \mathrm{H}), 1.46-1.51(\mathrm{~m}, 2 \mathrm{H}), 1.83$ (dt, $J=5.0$, $15.0 \mathrm{~Hz}, 1 \mathrm{H}), 1.94-2.02(\mathrm{~m}, 2 \mathrm{H}), 2.65-2.72(\mathrm{~m}, 1 \mathrm{H}), 2.86(\mathrm{dd}, J=1.5,9.5 \mathrm{~Hz}, 1 \mathrm{H}), 3.46$ (dt, $J=2.0,11.0$ $\mathrm{Hz}, 1 \mathrm{H}), 3.60-3.62(\mathrm{~m}, 1 \mathrm{H}), 3.71-3.75(\mathrm{~m}, 1 \mathrm{H}), 3.78-3.82(\mathrm{~m}, 2 \mathrm{H}), 5.00-5.09(\mathrm{~m}, 2 \mathrm{H}), 5.76-5.83(\mathrm{~m}, 1 \mathrm{H})$; ${ }^{13}$ C NMR (100 MHz, CDCl3) $\delta-5.3,-5.2,-4.9,-4.8,-3.5,-2.3,17.0,18.3,18.4,25.9,26.3,35.6,37.1$, 39.0, 59.7, 64.8, 67.0, 77.6, 85.1, 114.6, 140.9; IR (thin film) 2960, 2931, 2858, 1476, 1386, 1252, 1170, 1093, 1019, 909, 836, $775\left(\mathrm{~cm}^{-1}\right)$; HRMS (EI) $\mathrm{m} / \mathrm{z}$ calc'd for $\mathrm{C}_{29} \mathrm{H}_{62} \mathrm{O}_{4} \mathrm{Si}_{3} \mathrm{Na}\left(\mathrm{M}^{+}+\mathrm{Na}\right) 581.3848$, found 581.3834 .

Synthesis of (S)-2-((2S,3S,5R,6R)-3,5-bis(tert-butyldimethylsilyloxy)-6-(2-(tertbutyldimethylsilyloxy)ethyl)tetrahydro-2H-pyran-2-yl)propanal, 20:

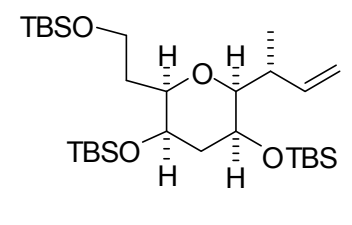

19

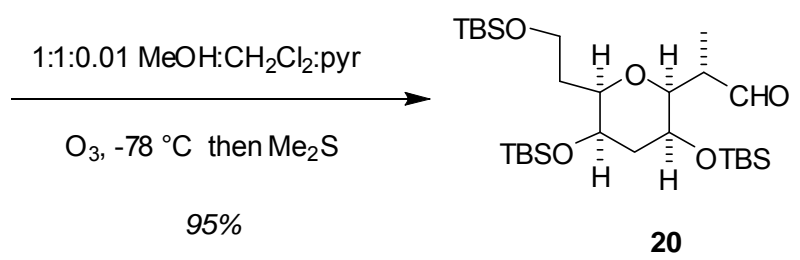

20

Alkene 19 (31 mg, $0.055 \mathrm{mmol}, 1.0 \mathrm{eq})$ was dissolved in 1:1 $\mathrm{MeOH}-\mathrm{CH}_{2} \mathrm{Cl}_{2}(1 \mathrm{~mL})$ and cooled to $-78^{\circ} \mathrm{C}$. Pyridine ( $60 \mu \mathrm{L}, 0.74 \mathrm{mmol}, 13.5$ eq.) was added. $\mathrm{O}_{3} / \mathrm{O}_{2}$ was bubbled through the solution for 5 minutes at $-78{ }^{\circ} \mathrm{C}$ at which point a steel blue color persisted. Stirring was continued for 5 minutes at $-78{ }^{\circ} \mathrm{C}$, then nitrogen was bubbled through the solution until the blue color dissipated. The reaction was warmed to room temperature and quenched with dimethyl sulfide $(200 \mu \mathrm{L})$ and stirring was continued for $12 \mathrm{~h}$. The solution was concentrated at reduced pressure, and the crude residue was purified by flash column chromatography (5\% EtOAc/Hexanes) to yield aldehyde $\mathbf{2 0}(29.5 \mathrm{mg}, 95 \%$ yield) as a clear colorless oil.

Data for 19: $[\alpha]_{\mathbf{D}}+13.8\left(c 1.0, \mathrm{CHCl}_{3}\right) ;{ }^{1} \mathbf{H}$ NMR $\left(500 \mathrm{MHz}, \mathrm{CDCl}_{3}\right) \delta 0.00(\mathrm{~d}, J=7.0 \mathrm{~Hz}, 6 \mathrm{H}), 0.03(\mathrm{~s}$, $6 \mathrm{H}), 0.06(\mathrm{~d}, J=3.0 \mathrm{~Hz}, 6 \mathrm{H}), 0.87(\mathrm{~s}, 9 \mathrm{H}), 0.88(\mathrm{~s}, 9 \mathrm{H}), 0.90(\mathrm{~s}, 9 \mathrm{H}), 1.18(\mathrm{~d}, J=7.5 \mathrm{~Hz}, 3 \mathrm{H}), 1.49-1.56$ (m, $1 \mathrm{H}), 1.88-2.00(\mathrm{~m}, 3 \mathrm{H}), 2.89-2.95(\mathrm{~m}, 1 \mathrm{H}), 3.51-3.54(\mathrm{~m}, 2 \mathrm{H}), 3.66-3.77(\mathrm{~m}, 3 \mathrm{H}), 3.91-3.93(\mathrm{~m}, 1 \mathrm{H})$, $9.73(\mathrm{~d}, J=0.5 \mathrm{~Hz}, 1 \mathrm{H}) ;{ }^{13} \mathbf{C}$ NMR $(100 \mathrm{MHz}, \mathrm{CDCl} 3) \delta-5.3,-5.2,-4.9,-4.8,-3.6,-3.2,10.7,18.2,18.3$, $18.4,25.9,26.2,26.3,35.1,38.3,47.3,59.5,65.1,66.9,79.7,203.5$; IR (thin film) 2960, 2931, 2854, 2703, $1725,1468,1386,1252,1162,1097,836,771\left(\mathrm{~cm}^{-1}\right)$; HRMS (EI) $\mathrm{m} / \mathrm{z}$ calc'd for $\mathrm{C}_{28} \mathrm{H}_{60} \mathrm{O}_{5} \mathrm{Si}_{3} \mathrm{Na}\left(\mathrm{M}^{+}+\mathrm{Na}\right)$ 583.3604, found 583.3624.

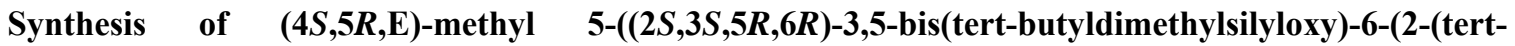
butyldimethylsilyloxy)ethyl)tetrahydro- $2 \mathrm{H}$-pyran-2-yl)-4-hydroxyhex-2-enoate, 21 and $(4 R, 5 R, \mathrm{E})$ Methyl 5-((2S,3S,5R,6R)-3,5-bis(tert-butyldimethylsilyloxy)-6-(2-(tert-butyldimethylsilyloxy)ethyl) tetrahydro-2H-pyran-2-yl)-4-hydroxyhex-2-enoate, epi-21:

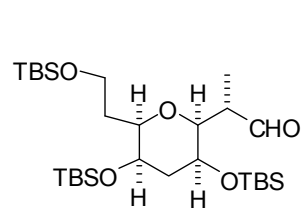

20

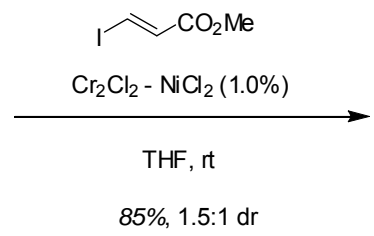

$85 \%, 1.5: 1 \mathrm{dr}$

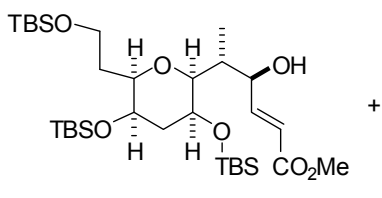

21

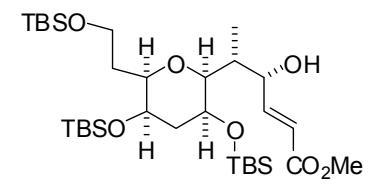

epi-21

Aldehyde 20 (1.97 g, $3.5 \mathrm{mmol})$ and trans- $\beta$-iodo-methylacrylate were dissolved in THF $(20 \mathrm{~mL})$ and stirred at room temperature under nitrogen. To this mixture was added $\mathrm{CrCl}_{2}$ containing $1 \% \mathrm{NiCl}_{2}$ by mass (ca. $1.5 \mathrm{~g}$ total). After $1 \mathrm{~h}$, additional $1 \% \mathrm{NiCl}_{2} / \mathrm{CrCl}_{2}$ (ca. $1 \mathrm{~g}$ ) was added to the pale green suspension, and 
the mixture was stirred for $18 \mathrm{~h}$ at room temperature. The reaction mixture was diluted with saturated $\mathrm{NH}_{4} \mathrm{Cl}(40 \mathrm{~mL})$, and diluted with EtOAc $(40 \mathrm{~mL})$. Ethylene diamine $(2 \mathrm{~mL})$ was added to this mixture and the resultant solution was stirred for $30 \mathrm{~min}$ before being treated $\mathrm{w} / 1 \mathrm{M} \mathrm{HCl}$ to $\mathrm{pH} 3$ to dissolve any emulsion. The solution was extracted with EtOAc $(3 \times 50 \mathrm{~mL})$ and the combined organics were dried with $\mathrm{Na}_{2} \mathrm{SO}_{4}$, filtered through a silica plug and concentrated. Silica gel chromatography of the resulting residue $\left(10 \% \mathrm{Et}_{2} \mathrm{O} / \mathrm{Hex}\right)$ provided the two diastereomeric products $(1.93 \mathrm{~g}, 85 \%$ combined yield, $\mathrm{dr}=2: 1)$. Further chromatography resulted in separation of the major, and desired, product $\left(\mathrm{R}_{\mathrm{f}}=0.15,10 \%\right.$ EtOAc/Hexanes $)$ to give $1.09 \mathrm{~g}$ of $21(57 \%)$.

Data for 21: $[\alpha]_{\mathbf{D}}+3.4\left(c 0.5, \mathrm{CHCl}_{3}\right) ;{ }^{1} \mathbf{H}$ NMR $\left(500 \mathrm{MHz}, \mathrm{CDCl}_{3}\right) \delta 0.26-0.82(\mathrm{~m}, 18 \mathrm{H}), 0.87(\mathrm{~s}, 9 \mathrm{H})$, $0.89(\mathrm{~s}, 18 \mathrm{H}), 0.98(\mathrm{~d}, J=7.0 \mathrm{~Hz}, 3 \mathrm{H}), 1.51-1.58(\mathrm{~m}, 1 \mathrm{H}), 1.83-1.90(\mathrm{~m}, 1 \mathrm{H}), 1.92-1.94(\mathrm{~m}, 2 \mathrm{H}), 2.05-2.10$ (m, $1 \mathrm{H}), 3.00(\mathrm{~d}, J=2.5 \mathrm{~Hz}, 1 \mathrm{H}), 3.53(\mathrm{dt}, J=3.0,12.5,1 \mathrm{H}), 3.60(\mathrm{t}, J=4.5 \mathrm{~Hz}, 1 \mathrm{H}), 3.68-3.72(\mathrm{~m}, 3 \mathrm{H})$, $3.73(\mathrm{~s}, 3 \mathrm{H}), 3.91-3.94(\mathrm{~m}, 1 \mathrm{H}), 4.50(\mathrm{br} \mathrm{s}, 1 \mathrm{H}), 6.10(\mathrm{dd}, J=2.0,15.5 \mathrm{~Hz}, 1 \mathrm{H}), 6.87$ (dd, $J=3.0,15.5 \mathrm{~Hz}$, $1 \mathrm{H}) ;{ }^{13} \mathrm{C}$ NMR $\left(100 \mathrm{MHz}, \mathrm{CDCl}_{3}\right) \delta-5.3,-5.2,-4.9,-3.8,-3.7,8.0,18.1,18.2,18.3,25.9,26.1,34.5,38.5$, $38.6,51.5,59.6,66.2,67.3,73.8,75.9,82.6,120.1,149.8,167.1$; IR (thin film) 3490, 2956, 2927, 2850, $2895,1725,1655,1472,1260,1170,1084,1019,913,832,771\left(\mathrm{~cm}^{-1}\right)$; HRMS (EI) $\mathrm{m} / \mathrm{z}$ calc'd for $\mathrm{C}_{32} \mathrm{H}_{66} \mathrm{O}_{7} \mathrm{Si}_{3} \mathrm{Na}\left(\mathrm{M}^{+}+\mathrm{Na}\right) 669.4008$, found 669.3986 .

Data for epi-21: $\left(\mathrm{R}_{\mathrm{f}}=0.20,10 \%\right.$ EtOAc/Hexanes $)[\alpha]_{\mathbf{D}}+9.7\left(\right.$ c $\left.0.5, \mathrm{CHCl}_{3}\right) ;{ }^{1} \mathbf{H} \mathbf{N M R}\left(500 \mathrm{MHz}, \mathrm{CDCl}_{3}\right)$ $\delta$ 0.02-0.06 (m, 18H), 0.88-0.90 (m, 27H), $1.22(\mathrm{~d}, J=7.0 \mathrm{~Hz}, 3 \mathrm{H}), 1.49-1.56(\mathrm{~m}, 1 \mathrm{H}), 1.81-1.92(\mathrm{~m}, 3 \mathrm{H})$, 2.01-2.04 (m, 1H), $3.22(\mathrm{~d}, J=6.5 \mathrm{~Hz}, 1 \mathrm{H}), 3.45-3.48(\mathrm{~m}, 1 \mathrm{H}), 3.52-3.53(\mathrm{~m}, 1 \mathrm{H}), 3.66-3.70(\mathrm{~m}, 3 \mathrm{H}), 3.74$ (s, 3H), 3.78-3.81 (m, 1H), $4.32(\mathrm{~d}, J=3.5 \mathrm{~Hz}, 1 \mathrm{H}), 6.15$ (dd, $J=2.0,15.5 \mathrm{~Hz}, 1 \mathrm{H}), 6.93(\mathrm{dd}, J=4.0,15.5$ $\mathrm{Hz}, 1 \mathrm{H}) ;{ }^{13} \mathbf{C}$ NMR $(100 \mathrm{MHz}, \mathrm{CDCl} 3) \delta-5.3,-5.2,-4.9,-4.8,-3.9,-3.8,12.6,18.1,18.2,18.3,25.9,26.1$, $34.6,38.6,39.5,51.5,59.7,66.9,67.1,75.2,75.8,76.6,79.1,120.7,150.6,167.1$; IR (thin film) 3486, 2956, 2927, 2858, 1729, 1659, 1464, 1390, 1256, 1166, 1097, 1023, 909, $771\left(\mathrm{~cm}^{-1}\right)$; HRMS (EI) $\mathrm{m} / \mathrm{z}$ calc'd for $\mathrm{C}_{32} \mathrm{H}_{66} \mathrm{O}_{7} \mathrm{Si}_{3} \mathrm{Na}\left(\mathrm{M}^{+}+\mathrm{Na}\right) 669.4008$, found 669.3985 .

Synthesis of $(4 S, 5 R, E)$-Methyl 5-((2S,3S,5R,6R)-3,5-bis(tert-butyldimethylsilyloxy)-6-(2-(tertbutyldimethylsilyloxy)ethyl)tetrahydro-2H-pyran-2-yl)-4-(4-methoxybenzyloxy)hex-2-enoate, 22:

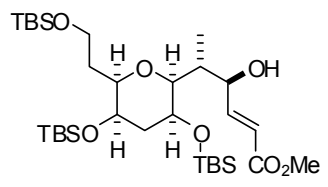

21

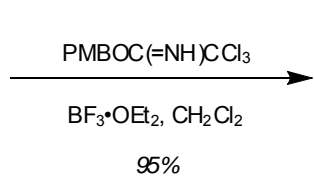

$95 \%$

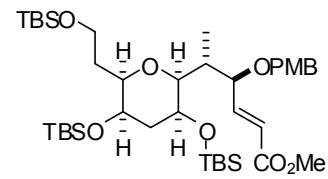

22

Alcohol 21 (811 mg, $1.25 \mathrm{mmol}, 1.0 \mathrm{eq})$ and p-methoxybenzyltrichloroacetimidate $(3.54 \mathrm{~g}, 12.5 \mathrm{mmol}$, 10.0 eq) were dissolved in $\mathrm{CH}_{2} \mathrm{Cl}_{2}$ and cooled to $0{ }^{\circ} \mathrm{C}$. To this solution was added $\mathrm{BF}_{3}-\mathrm{OEt}_{2}(157 \mu \mathrm{L}, 1.25$ mmol, 1 eq) dropwise and the resulting solution was stirred for $1 \mathrm{~h}$ at $0{ }^{\circ} \mathrm{C}$. The reaction mixture was then carefully poured into cold saturated aqueous $\mathrm{NaHCO}_{3}$. The layers were separated, and the aqueous layer was extracted with $\mathrm{CH}_{2} \mathrm{Cl}_{2}$. The combined organic phase was washed with saturated aqueous $\mathrm{NaHCO}_{3}$ and brine, dried over $\mathrm{Na}_{2} \mathrm{SO}_{4}$, filtered and concentrated. The crude residue was purified by FCC $(10 \%$ EtOAc/Hexanes) to yield the title compound, 22 (911 mg, 95\% yield) as clear oil.

Data for XXX: $[\alpha]_{\mathbf{D}}+30.6\left(c \quad 0.5, \mathrm{CHCl}_{3}\right) ;{ }^{1} \mathbf{H}$ NMR $\left(500 \mathrm{MHz}, \mathrm{CDCl}_{3}\right) \delta-0.06(\mathrm{~s}, 3 \mathrm{H}), 0.02-0.04(\mathrm{~m}$, $15 \mathrm{H}), 0.87-0.89(\mathrm{~m}, 27 \mathrm{H}), 0.98(\mathrm{~d}, J=7.0 \mathrm{~Hz}, 3 \mathrm{H}), 1.45-1.50(\mathrm{~m}, 1 \mathrm{H}), 1.84-1.95(\mathrm{~m}, 3 \mathrm{H}), 2.07-2.12$ (m, $1 \mathrm{H}), 3.16$ (dd, $J=2.0,8.5 \mathrm{~Hz}, 1 \mathrm{H}), 3.36$ (br s, $1 \mathrm{H}), 3.40$ (dt, $J=2.0,10.5 \mathrm{~Hz}, 1 \mathrm{H}), 3.57-3.59$ (m, $1 \mathrm{H}), 3.71-$ $3.74(\mathrm{~m}, 2 \mathrm{H}), 3.76(\mathrm{~s}, 3 \mathrm{H}), 3.81(\mathrm{~s}, 3 \mathrm{H}), 3.83-3.95(\mathrm{~m}, 1 \mathrm{H}), 4.14(\mathrm{~d}, J=12.0 \mathrm{~Hz}, 1 \mathrm{H}), 4.57(\mathrm{~d}, J=12.0 \mathrm{~Hz}$, $1 \mathrm{H}), 6.04(\mathrm{dd}, J=1.5,17.0 \mathrm{~Hz}, 1 \mathrm{H}), 6.83(\mathrm{dd}, J=5.0,16.0 \mathrm{~Hz}, 1 \mathrm{H}), 6.87(\mathrm{~d}, J=8.5 \mathrm{~Hz}, 2 \mathrm{H}), 7.20(\mathrm{~d}, J=$ $8.5 \mathrm{~Hz}, 2 \mathrm{H}) ;{ }^{13} \mathbf{C}$ NMR $\left(100 \mathrm{MHz}, \mathrm{CDCl}_{3}\right) \delta-5.2,-5.1,-4.8,-3.5,-2.8,9.9,18.1,18.2,18.4,25.9,26.2$, $26.3,35.4,37.4,38.8,51.6,55.3,60.0,64.9,66.9,70.4,76.5,77.5,80.4,113.7,121.7,129.5,130.2,147.5$, 159.3, 166.7; IR (thin film) 2956, 2923, 2858, 1745, 1517, 1460, 1252, 1097, 836, $766\left(\mathrm{~cm}^{-1}\right)$; HRMS (EI) $\mathrm{m} / \mathrm{z}$ calc'd for $\mathrm{C}_{40} \mathrm{H}_{74} \mathrm{O}_{8} \mathrm{Si}_{3} \mathrm{Na}\left(\mathrm{M}+\mathrm{Na}^{+}\right)$789.4583, found 789.4550 . 
Synthesis of $(4 S, 5 R, E)$-Methyl 5-((5aR,7S,8S,9aR)-8-hydroxy-2,2-dimethylhexahydro-4H-pyrano[3,2d][1,3]dioxepin-7-yl)-4-(4-methoxybenzyloxy)hex-2-enoate, 23:
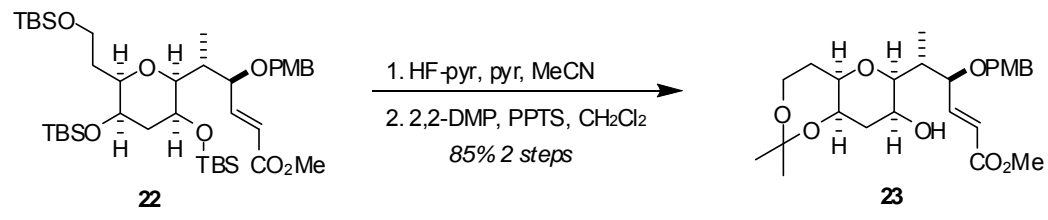

To a solution of $p$-methoxybenzyl ether $22(628 \mathrm{mg}, 0.81 \mathrm{mmol})$ in acetonitrile $(16 \mathrm{~mL})$ at $0{ }^{\circ} \mathrm{C}$ was added pyridine $(318 \mu \mathrm{L})$ followed by the slow addition of HF-pyridine reagent $(3.18 \mathrm{~mL})$. The reaction was stirred for $1 \mathrm{~h}$ at $0{ }^{\circ} \mathrm{C}$ at which time TLC analysis showed no remaining starting material. The reaction was cautiously quenched with sat'd aq. $\mathrm{NaHCO}_{3}(100 \mathrm{~mL})$ and diluted with EtOAc $(100 \mathrm{~mL})$. The layers were separated and the aqueous layer was extracted with EtOAc $(3 \mathrm{X} 50 \mathrm{~mL})$. The combined organics were washed with sat'd aq. $\mathrm{NaHCO}_{3}$, dried $\mathrm{Na}_{2} \mathrm{SO}_{4}$, filtered and concentrated. The crude residue was used in the next reaction without further purification.

The crude triol from above and 2,2-dimethoxypropane (200 $\mu \mathrm{L}, 1.62 \mathrm{mmol})$ were dissolved in $\mathrm{CH}_{2} \mathrm{Cl}_{2}(6.2$ $\mathrm{mL}$ ). To this solution was added pyridinium $p$-toluenesulfonate $(3 \mathrm{mg})$ and the reaction was stirred at room temperature for $12 \mathrm{~h}$. The reaction was quenched with sat'd aq. $\mathrm{NaHCO}_{3}(10 \mathrm{~mL})$, the layers were separated and the aqueous layer was extracted with $\mathrm{CH}_{2} \mathrm{Cl}_{2}(3 \times 25 \mathrm{~mL})$. The combined organics were washed with brine, dried $\mathrm{Na}_{2} \mathrm{SO}_{4}$. filtered and concentrated. The crude residue was purified by FCC (EtOAc/Hexanes/Et ${ }_{3} \mathrm{~N} \mathrm{1:1:0.001)} \mathrm{to} \mathrm{yield} \mathrm{acetonide} 23$ as a clear oil (319 mg, 85\% over 2 steps).

Data for 23: $[\alpha]_{\mathbf{D}}+15.1\left(\right.$ c $\left.1.0, \mathrm{CHCl}_{3}\right) ;{ }^{1} \mathbf{H}$ NMR $\left(500 \mathrm{MHz}, \mathrm{CDCl}_{3}\right) \delta 1.01(\mathrm{~d}, J=6.8 \mathrm{~Hz}, 3 \mathrm{H}), 1.32(\mathrm{~s}$, $3 \mathrm{H}), 1.33(\mathrm{~s}, 3 \mathrm{H}), 1.66(\mathrm{dt}, J=2.8,11.6 \mathrm{~Hz}, 1 \mathrm{H}), 1.74-1.77(\mathrm{~m}, 2 \mathrm{H}), 1.98(\mathrm{dt}, J=3.2,14.4 \mathrm{~Hz}, 1 \mathrm{H}), 2.07-$ $2.11(\mathrm{~m}, 1 \mathrm{H}), 3.24(\mathrm{~d}, J=8.4 \mathrm{~Hz}, 1 \mathrm{H}), 3.29-3.34(\mathrm{~m}, 1 \mathrm{H}), 3.40-3.47(\mathrm{~m}, 2 \mathrm{H}), 3.57(\mathrm{~d}, J=11.2 \mathrm{~Hz}, 1 \mathrm{H})$, $3.75(\mathrm{~s}, 3 \mathrm{H}), 3.79(\mathrm{~s}, 3 \mathrm{H}), 3.96-4.02(\mathrm{~m}, 2 \mathrm{H}), 4.17-4.20(\mathrm{~m}, 2 \mathrm{H}), 4.53(\mathrm{~d}, J=11.6 \mathrm{~Hz}, 1 \mathrm{H}), 6.05(\mathrm{dd}, J=$ $1.2,15.6 \mathrm{~Hz}, 1 \mathrm{H}), 6.86(\mathrm{~d}, J=8.8 \mathrm{~Hz}, 2 \mathrm{H}), 6.95(\mathrm{dd}, J=5.2,16.0 \mathrm{~Hz}, 1 \mathrm{H}), 7.20(\mathrm{~d}, J=8.4 \mathrm{~Hz}, 2 \mathrm{H}) ;{ }^{13} \mathbf{C}$ NMR $\left(100 \mathrm{MHz}, \mathrm{CDCl}_{3}\right) \delta 10.4,24.3,25.2,36.1,36.4,38.4,51.5,55.2,58.0,64.9,67.9,70.5,76.2,82.0$, $100.8,113.7,121.3,129.3,130.2,148.2,159.2,166.7$; IR (thin film) 3510, 2988, 2939, 2838, 1725, 1614, $1517,1435,1382,1248,1211,1158,1052,1003,824\left(\mathrm{~cm}^{-1}\right)$; HRMS (EI) $\mathrm{m} / \mathrm{z}$ calc'd for $\mathrm{C}_{25} \mathrm{H}_{36} \mathrm{O}_{8} \mathrm{Na}$ $\left(\mathrm{M}+\mathrm{Na}^{+}\right)$487.2302, found 487.2286.

\section{Synthesis of Pyranopyran 6:}
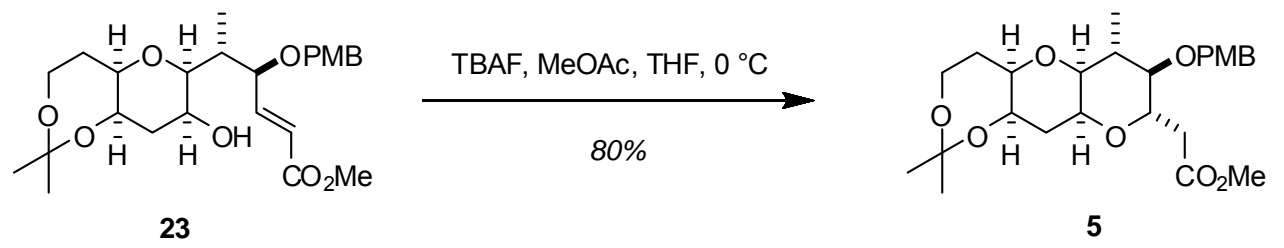

To a stirring solution of acetonide $23(717 \mathrm{mg}, 1.54 \mathrm{mmol})$ in THF $(46 \mathrm{~mL})$ and methyl acetate $(5.1 \mathrm{~mL})$ at $0{ }^{\circ} \mathrm{C}$ was added TBAF $(11.5 \mathrm{~mL}, 11.5 \mathrm{mmol}, 1.0 \mathrm{M}$ in THF). After $1 \mathrm{~h}$, additional methyl acetate $(5.1 \mathrm{~mL})$ and TBAF $\left(5.7 \mathrm{~mL}, 5.7 \mathrm{mmol}, 1.0 \mathrm{M}\right.$ in THF) were added and the reaction was stirred at $0{ }^{\circ} \mathrm{C}$ for $12 \mathrm{~h}$. The reaction was quenched with sat'd aq. $\mathrm{NaHCO}_{3}(75 \mathrm{~mL})$ and extracted with EtOAc $(3 \times 75 \mathrm{~mL})$. The combined organics were washed with $\mathrm{H}_{2} \mathrm{O}(50 \mathrm{~mL})$ and brine $(50 \mathrm{~mL})$, dried $\mathrm{Na}_{2} \mathrm{SO}_{4}$, filtered and concentrated. The crude residue was purified by FCC $\left(1: 9: 0.001 \mathrm{EtOAc} / \mathrm{Hex} / \mathrm{Et}_{3} \mathrm{~N}\right)$ to yield recovered starting material $23(95 \mathrm{mg})$ and the cyclized product $5(572 \mathrm{mg}, 80 \%)$ as clear oils.

Data for 5: $[\alpha]_{\mathbf{D}}-41.2\left(c 1.0, \mathrm{CHCl}_{3}\right) ;{ }^{1} \mathbf{H}$ NMR $\left(400 \mathrm{MHz}, \mathrm{CDCl}_{3}\right) \delta 1.08(\mathrm{~d}, J=9.5 \mathrm{~Hz}, 3 \mathrm{H}), 1.32(\mathrm{~s}$, $3 \mathrm{H}), 1.36(\mathrm{~s}, 3 \mathrm{H}), 1.71-1.80(\mathrm{~m}, 2 \mathrm{H}), 1.83-1.90(\mathrm{~m}, 1 \mathrm{H}), 2.02-2.08(\mathrm{~m}, 1 \mathrm{H}), 2.11-2.19(\mathrm{~m}, 1 \mathrm{H}), 2.47-2.57$ (m, 2H), 3.17 (dd, $J=5.0,11.5 \mathrm{~Hz}, 1 \mathrm{H}), 3.43-3.49(\mathrm{~m}, 3 \mathrm{H}), 3.55(\mathrm{~s}, 3 \mathrm{H}), 3.79(\mathrm{~s}, 3 \mathrm{H}), 3.83-3.85(\mathrm{~m}, 1 \mathrm{H})$, 3.88-3.90 (m, 1H), 3.99-4.05 (m, 1H), 4.41 (d, $J=14.0 \mathrm{~Hz}, 1 \mathrm{H}), 4.56(\mathrm{~d}, J=14.0 \mathrm{~Hz}, 1 \mathrm{H}), 4.66$ (dt, $J=$ 
7.5, $19.5 \mathrm{~Hz}, 1 \mathrm{H}), 6.85(\mathrm{~d}, J=11.0 \mathrm{~Hz}, 2 \mathrm{H}), 7.26(\mathrm{~d}, J=11.0 \mathrm{~Hz}, 2 \mathrm{H}) ;{ }^{13} \mathbf{C}$ NMR $(100 \mathrm{MHz}$, $\left.\mathrm{CDCl}_{3}\right) \delta 18.1,24.2,24.9,34.6,35.6,38.6,38.9,51.3,55.2,58.1,65.1,65.6,69.1,71.1,75.2,79.1,82.2$, 100.6, 113.5, 129.3, 130.6, 159.0, 171.6; IR (thin film) 2982, 2939, 2904, 2866, 2831, 1735, 1607, 1464, $1444,1374,1246,1215,1095,1056,827\left(\mathrm{~cm}^{-1}\right)$; HRMS (EI) $\mathrm{m} / \mathrm{z}$ calc'd for $\mathrm{C}_{25} \mathrm{H}_{36} \mathrm{O}_{8} \mathrm{Na}\left(\mathrm{M}+\mathrm{Na}^{+}\right)$ 487.2302, found 487.2295. 
Henderson, Jackson, Phillips SI 12

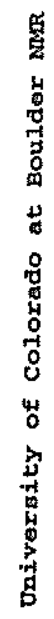

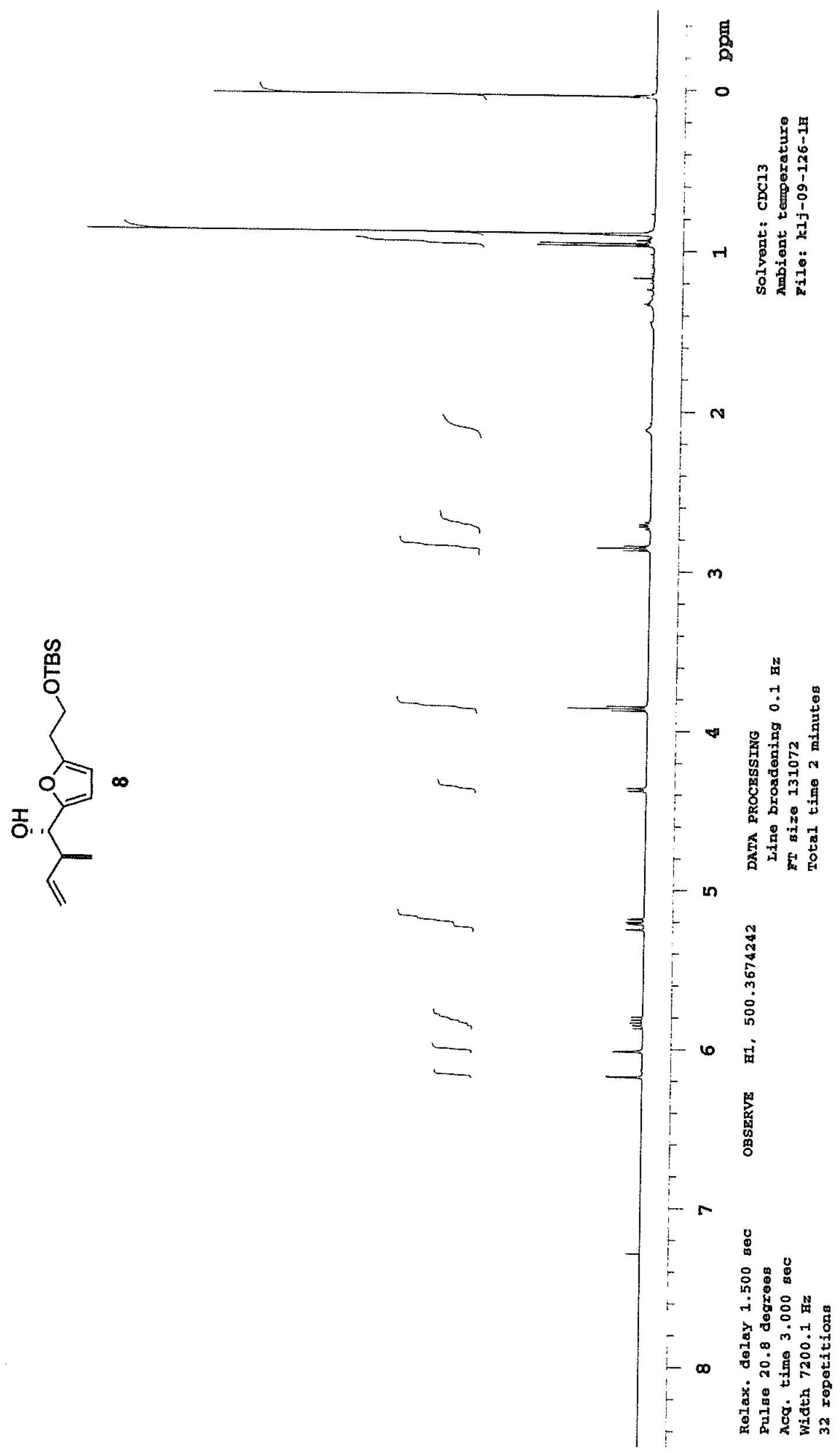


Henderson, Jackson, Phillips SI 13

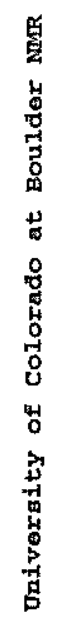

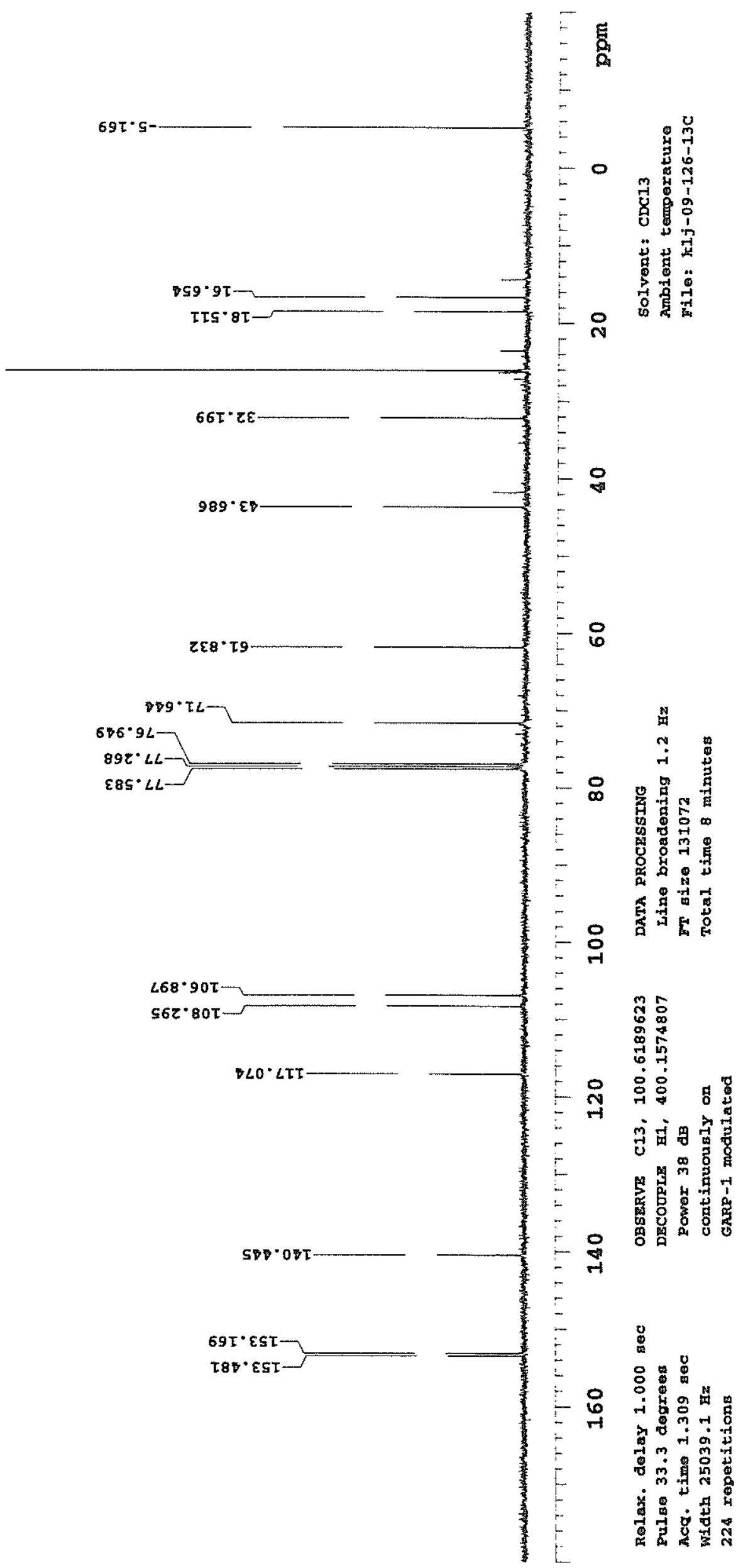


Henderson, Jackson, Phillips SI 14

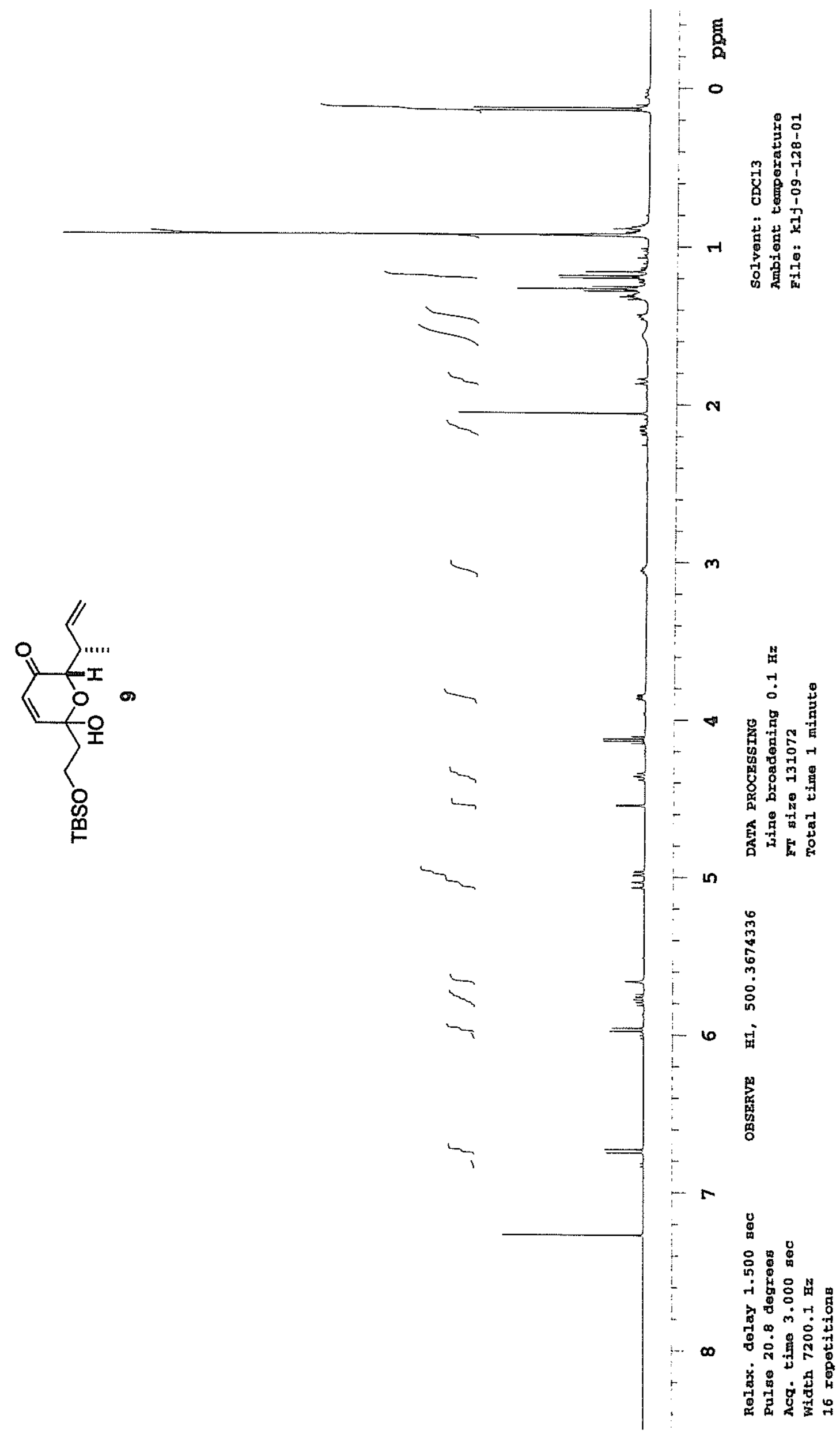


Henderson, Jackson, Phillips SI 15

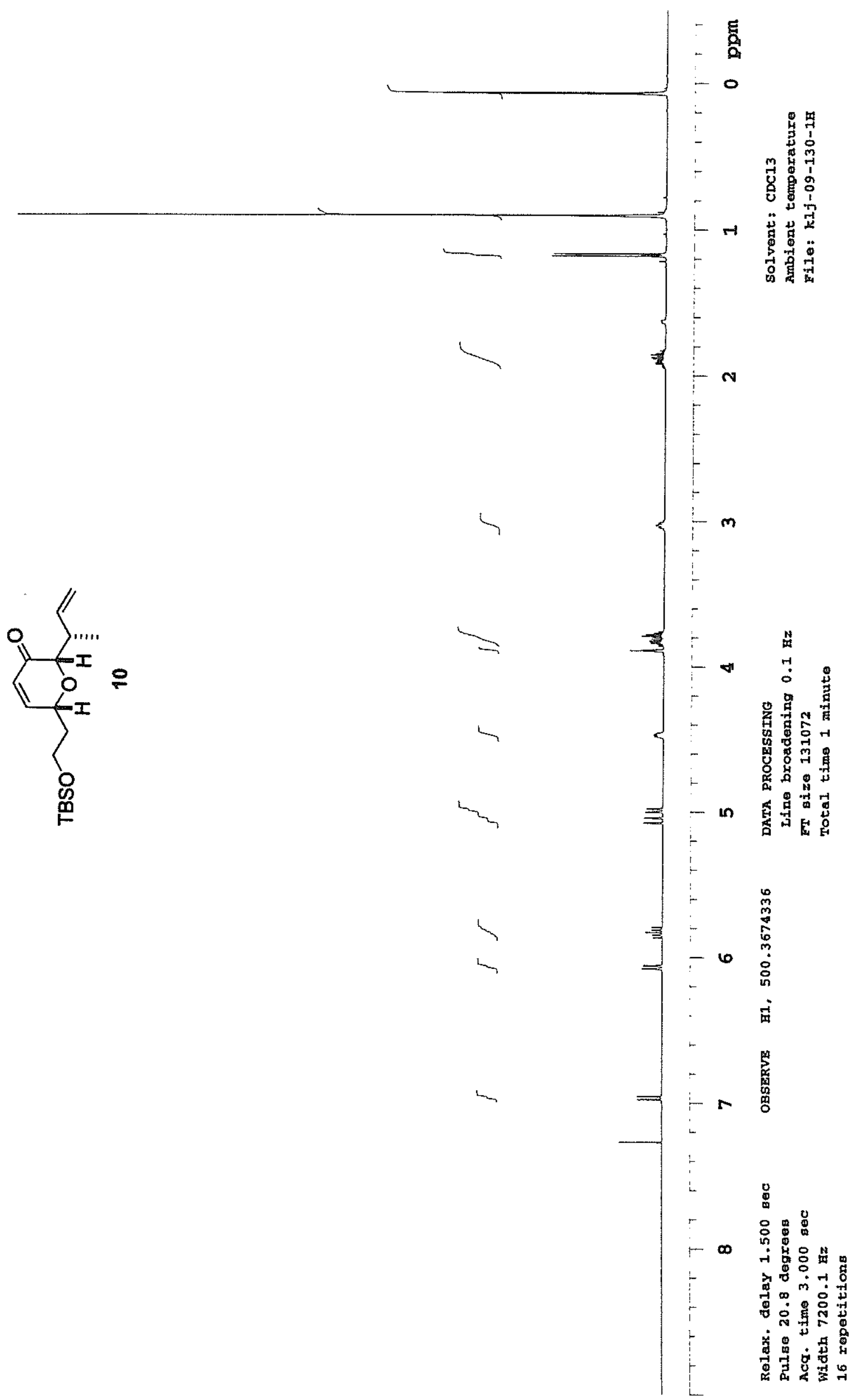


Henderson, Jackson, Phillips SI 16

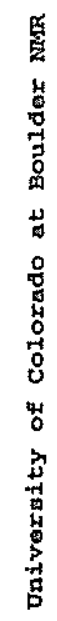

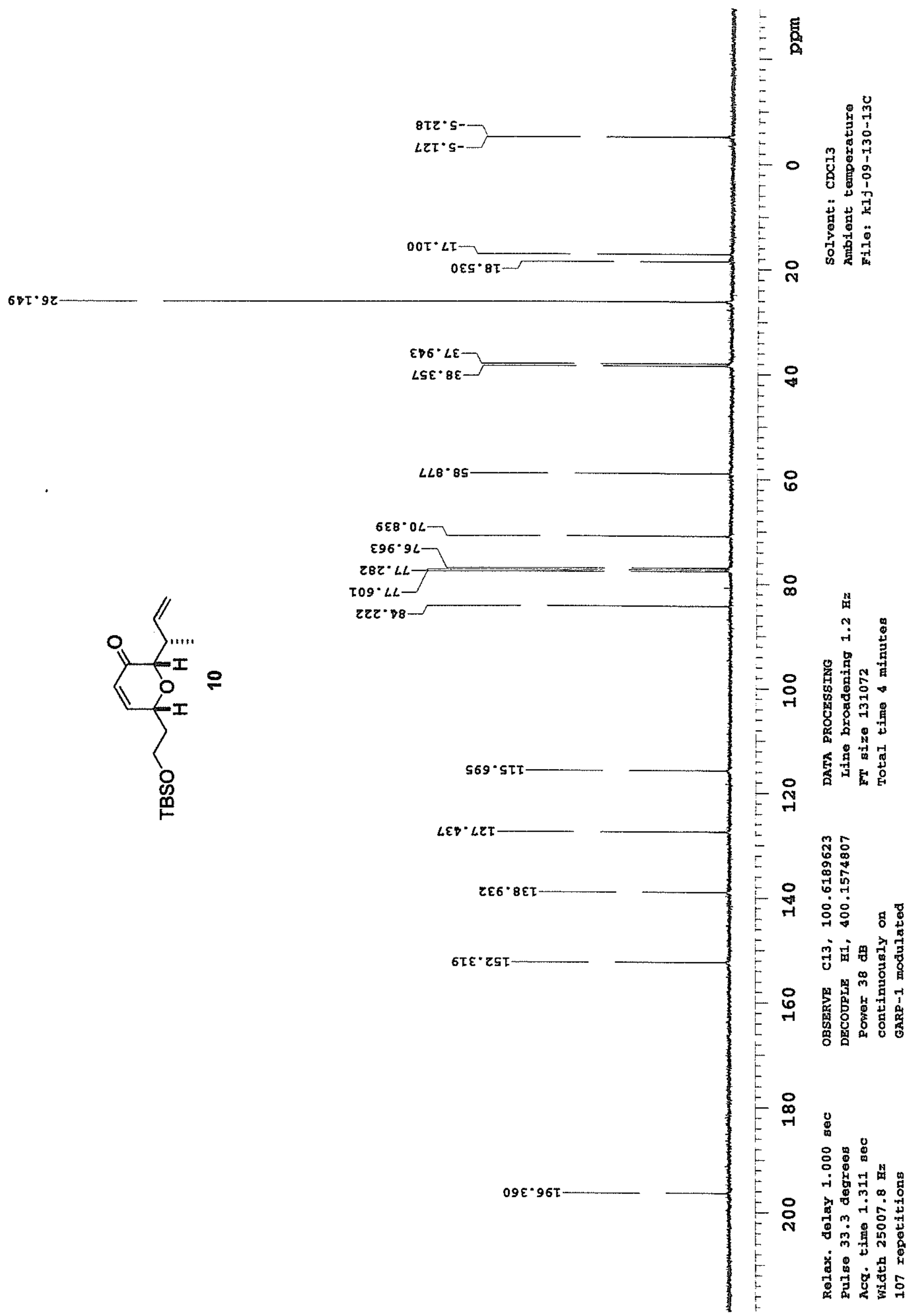


Henderson, Jackson, Phillips SI 17

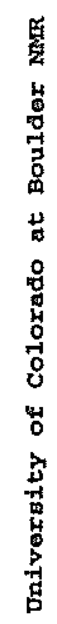
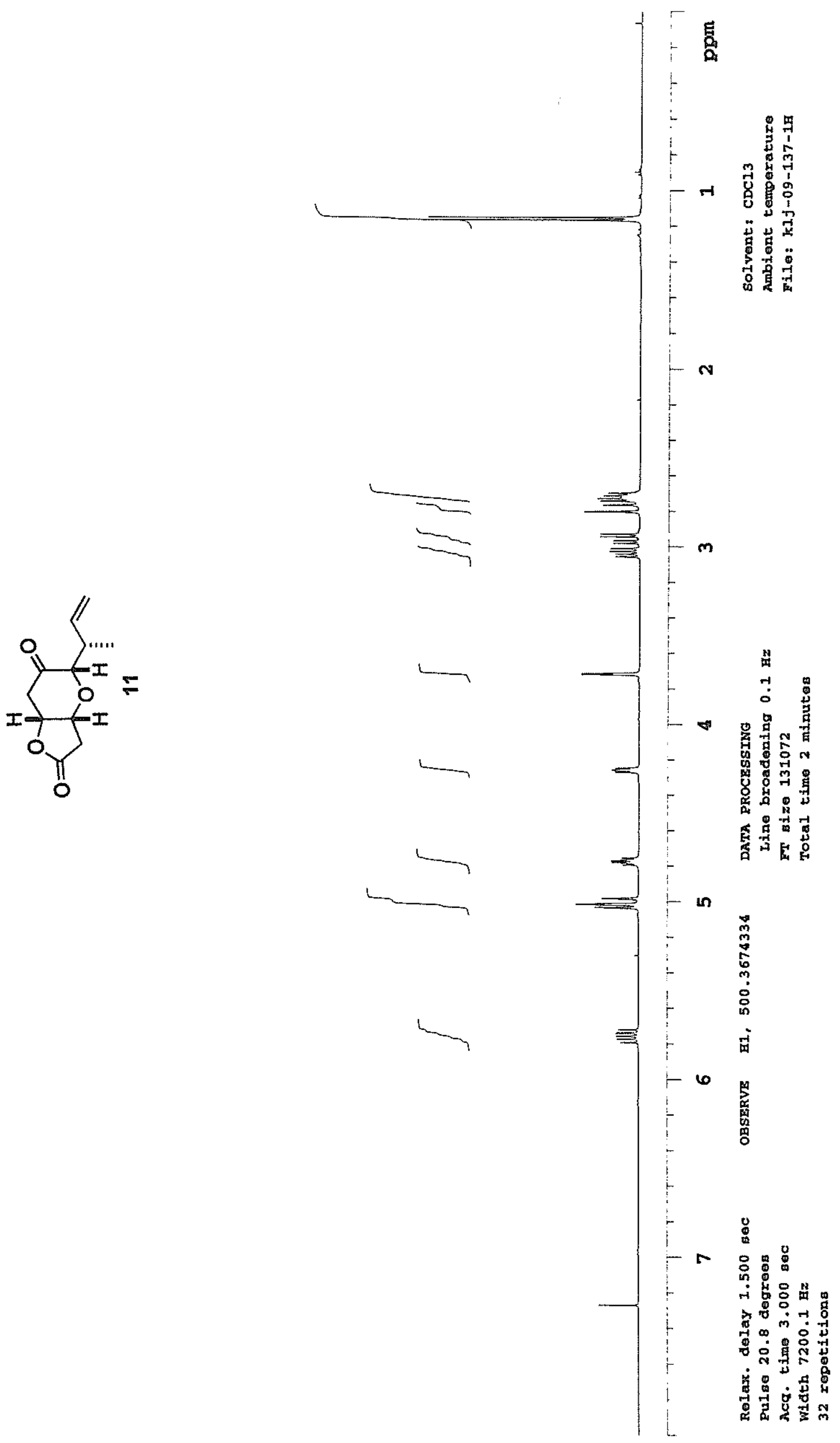
Henderson, Jackson, Phillips SI 18

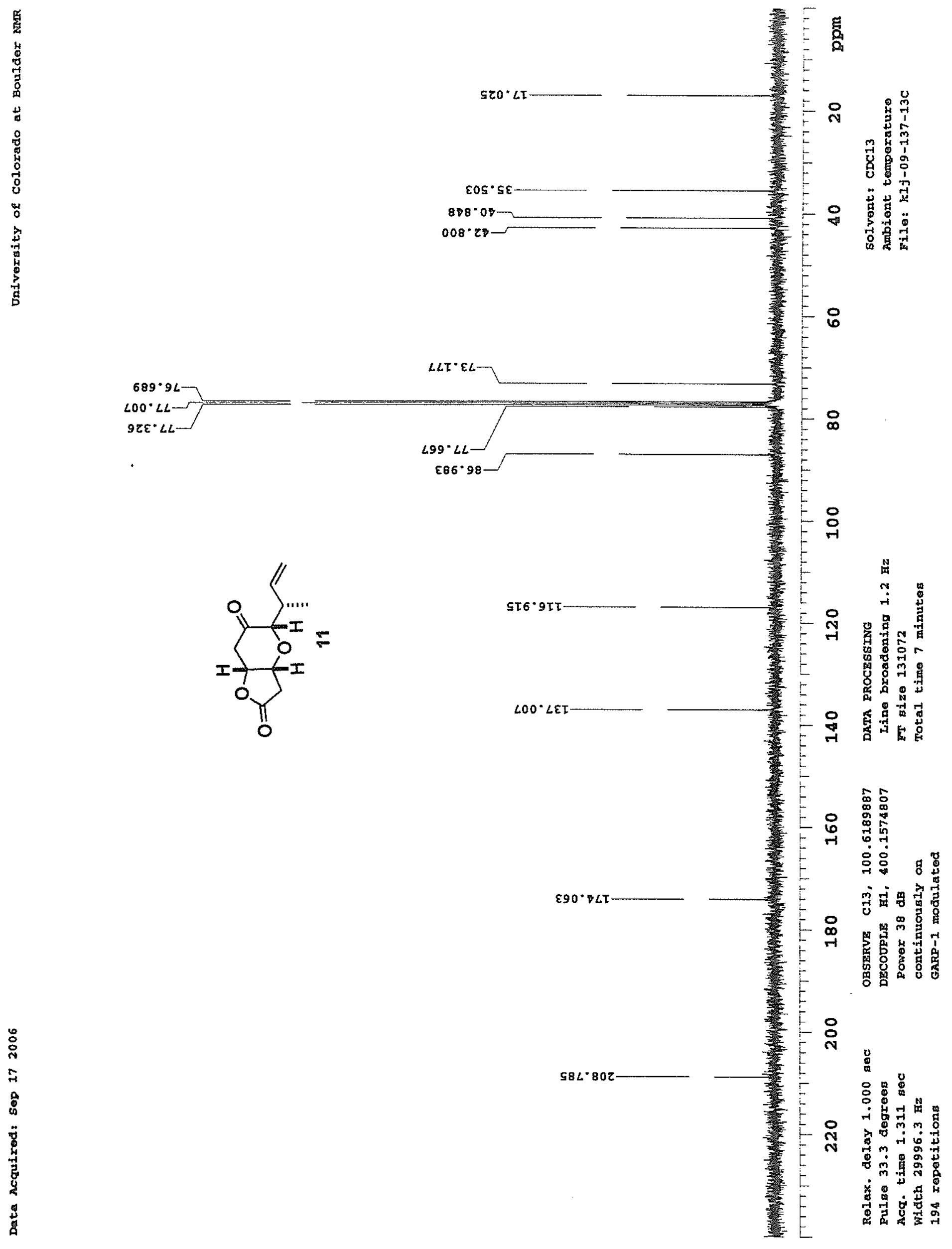


Henderson, Jackson, Phillips SI 19

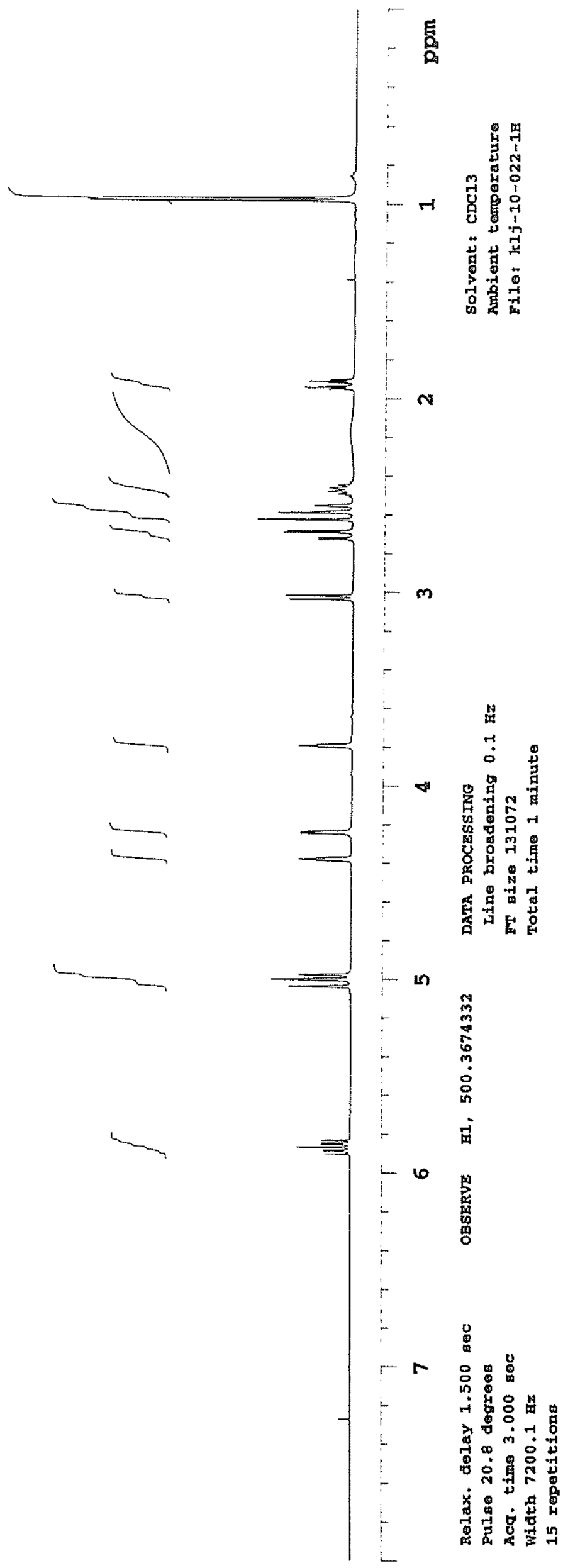


Henderson, Jackson, Phillips SI 20
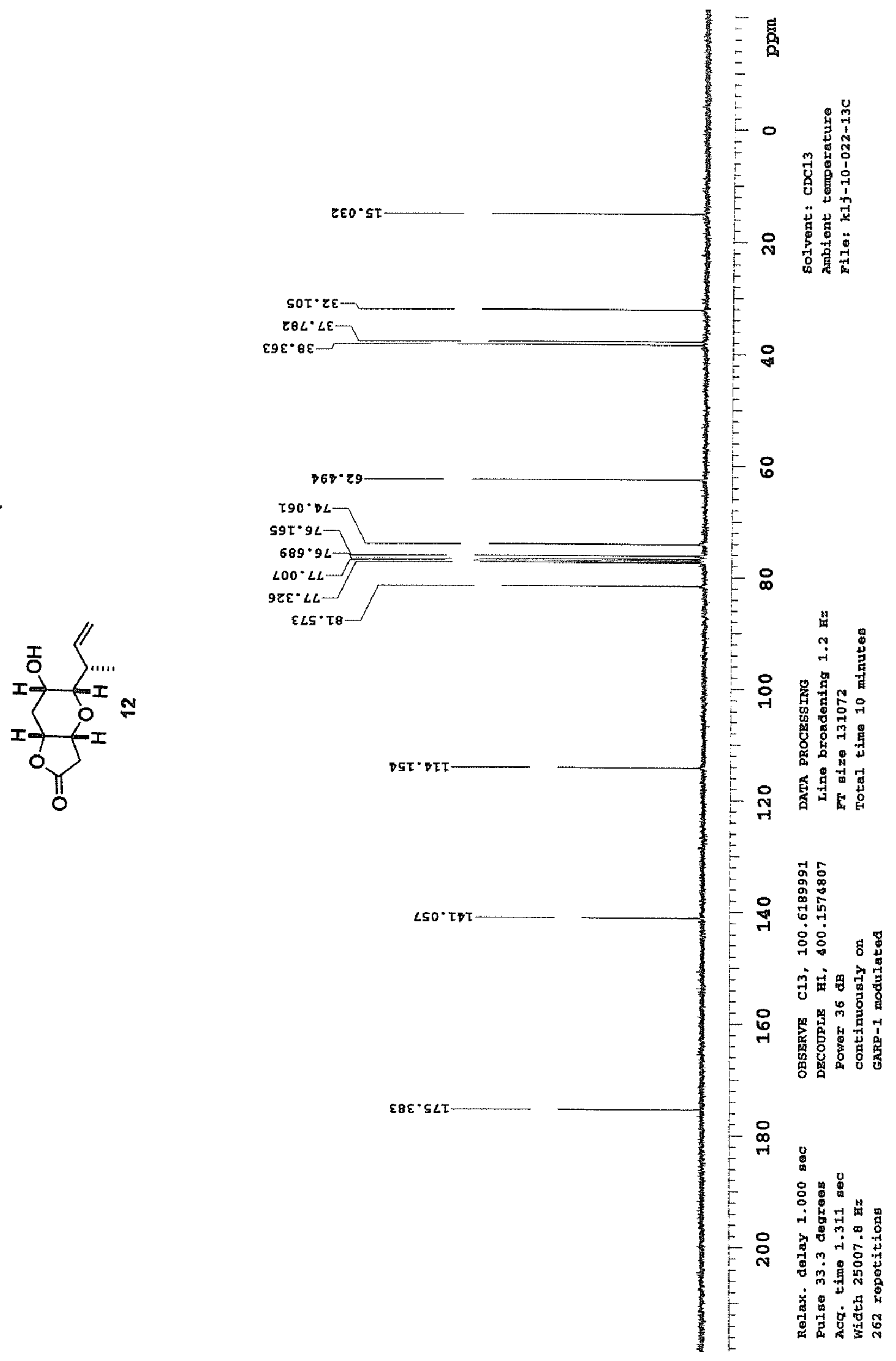
Henderson, Jackson, Phillips SI 21

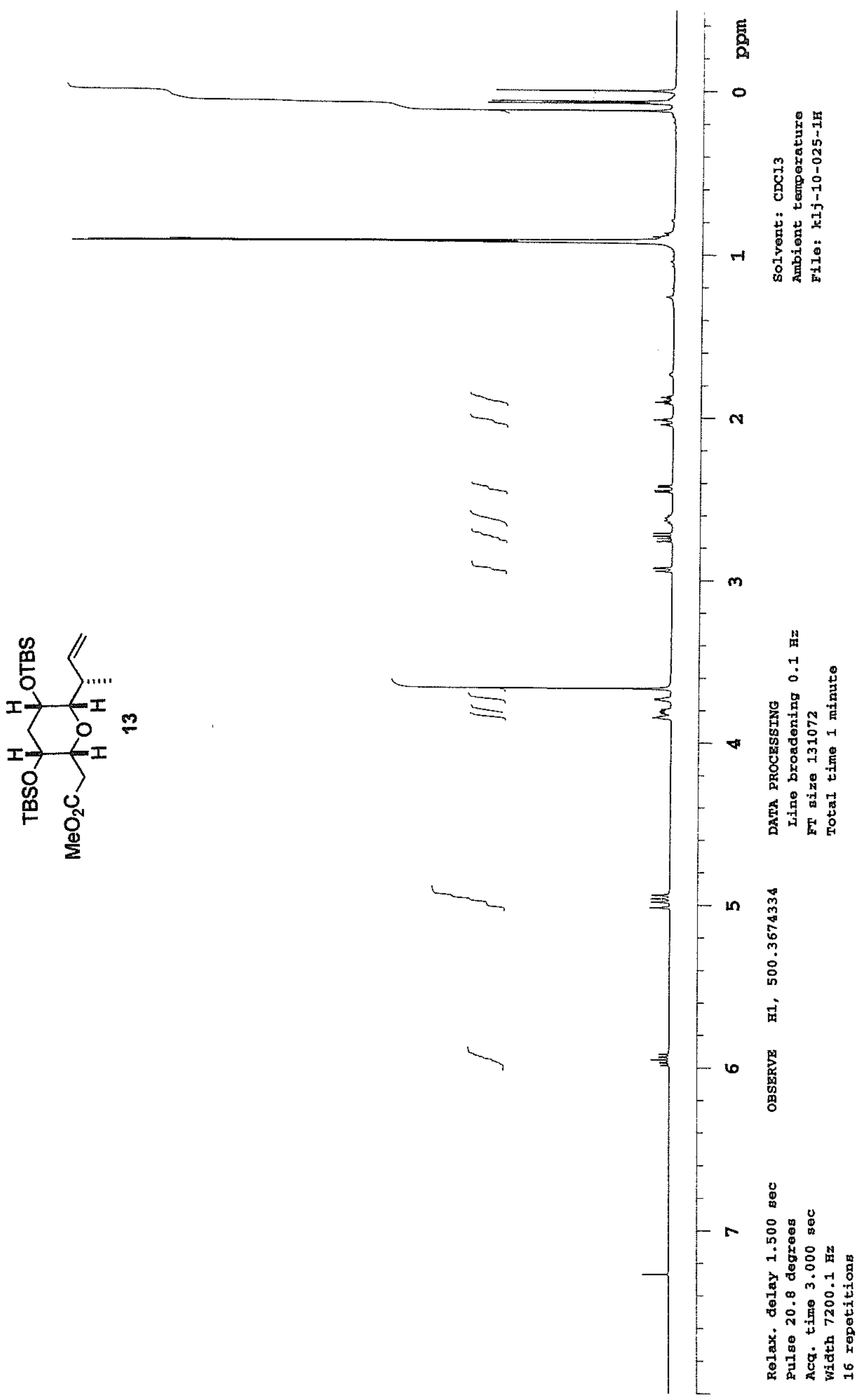


Henderson, Jackson, Phillips SI 22
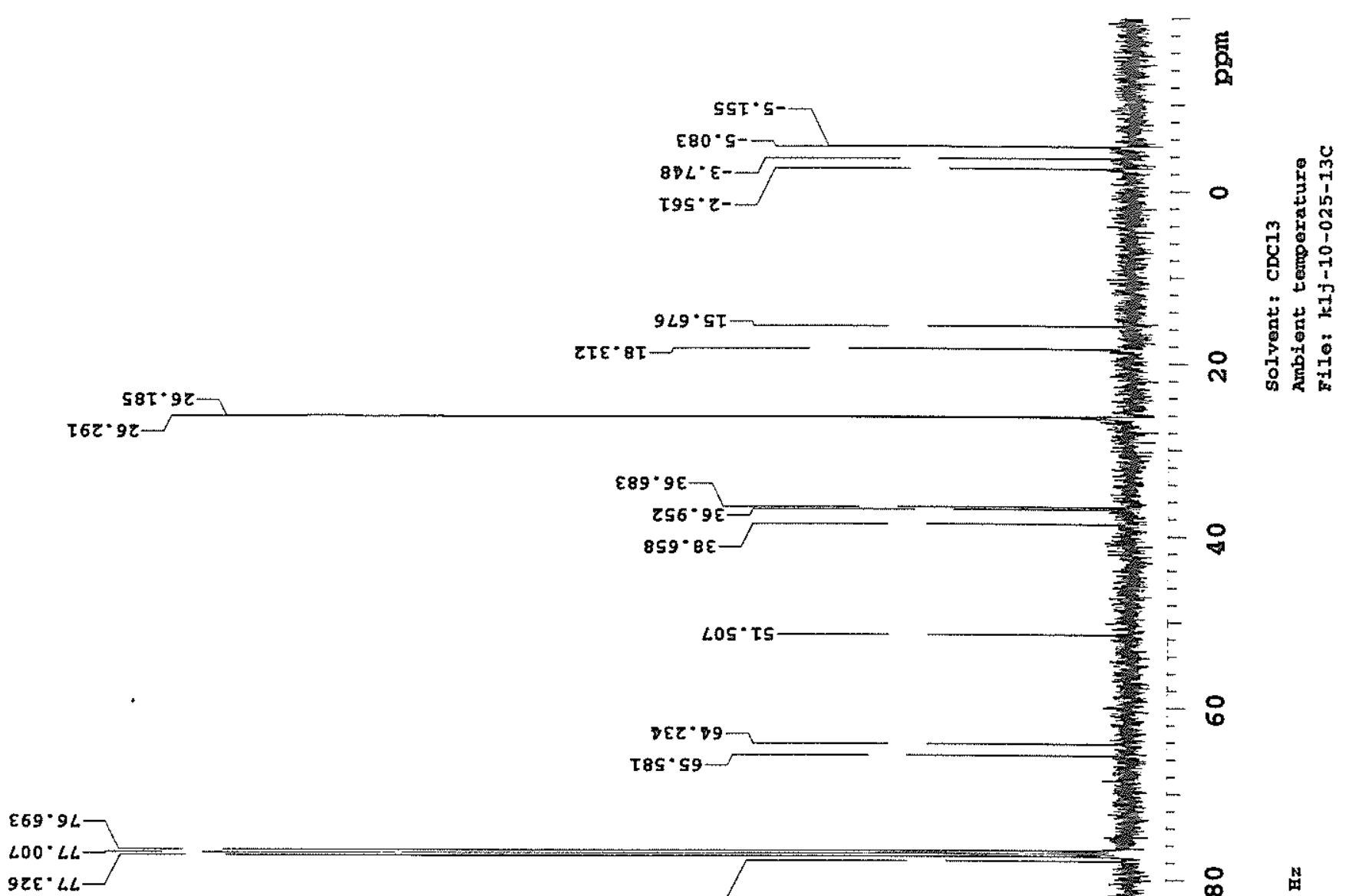

$58 \mathrm{~T} \cdot 9 \mathrm{z}$

$\tau 6 \tau \cdot 9 \tau-$
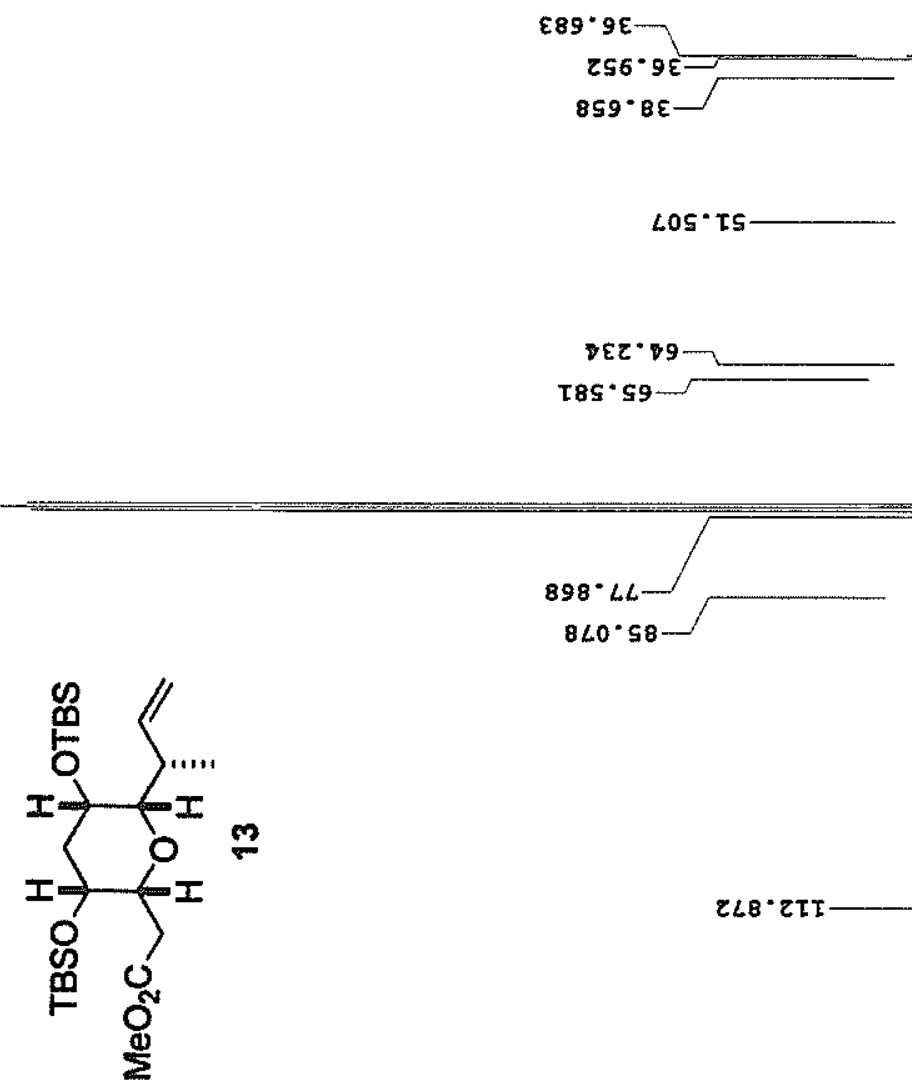

웅

8
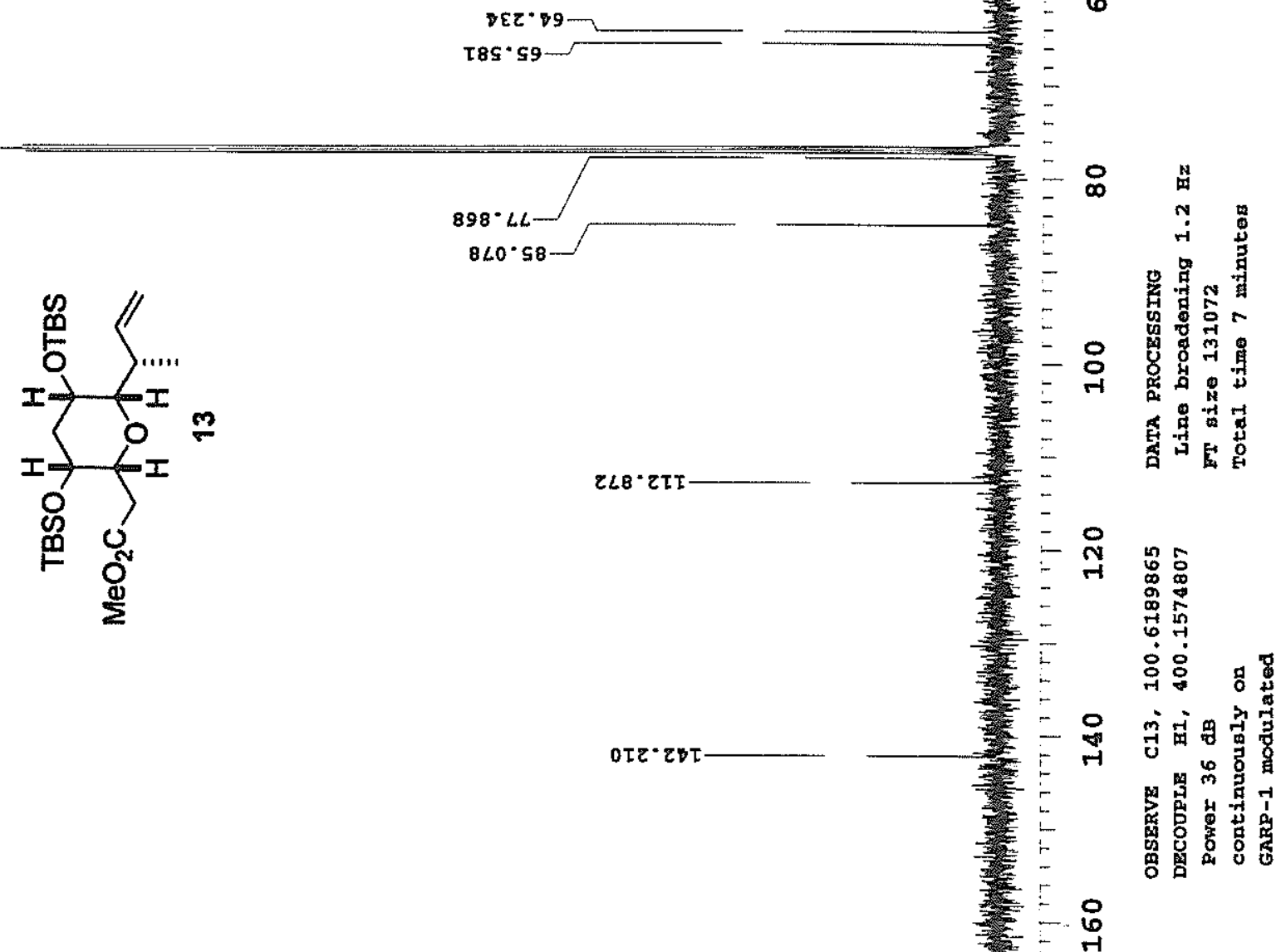
Henderson, Jackson, Phillips SI 23

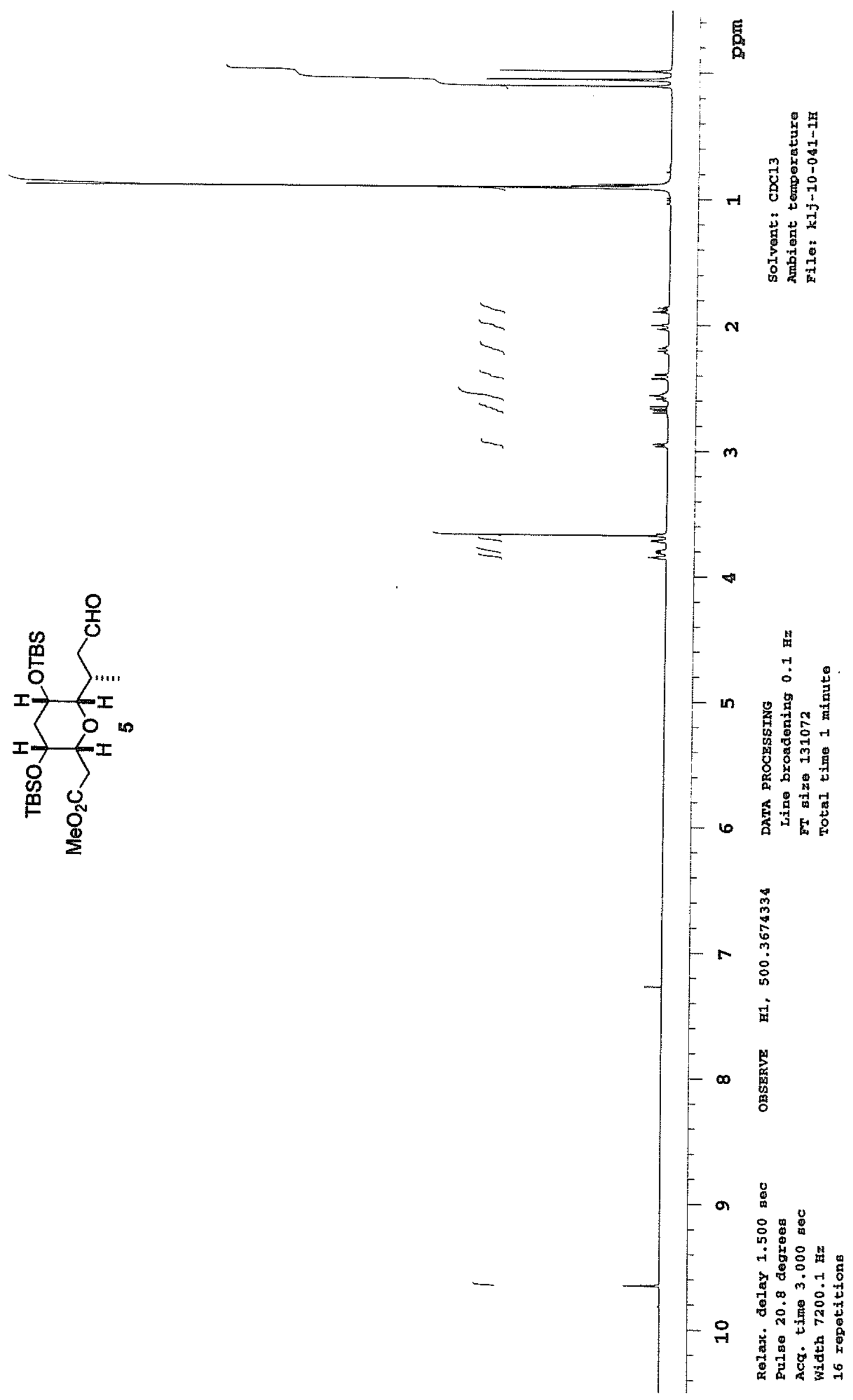




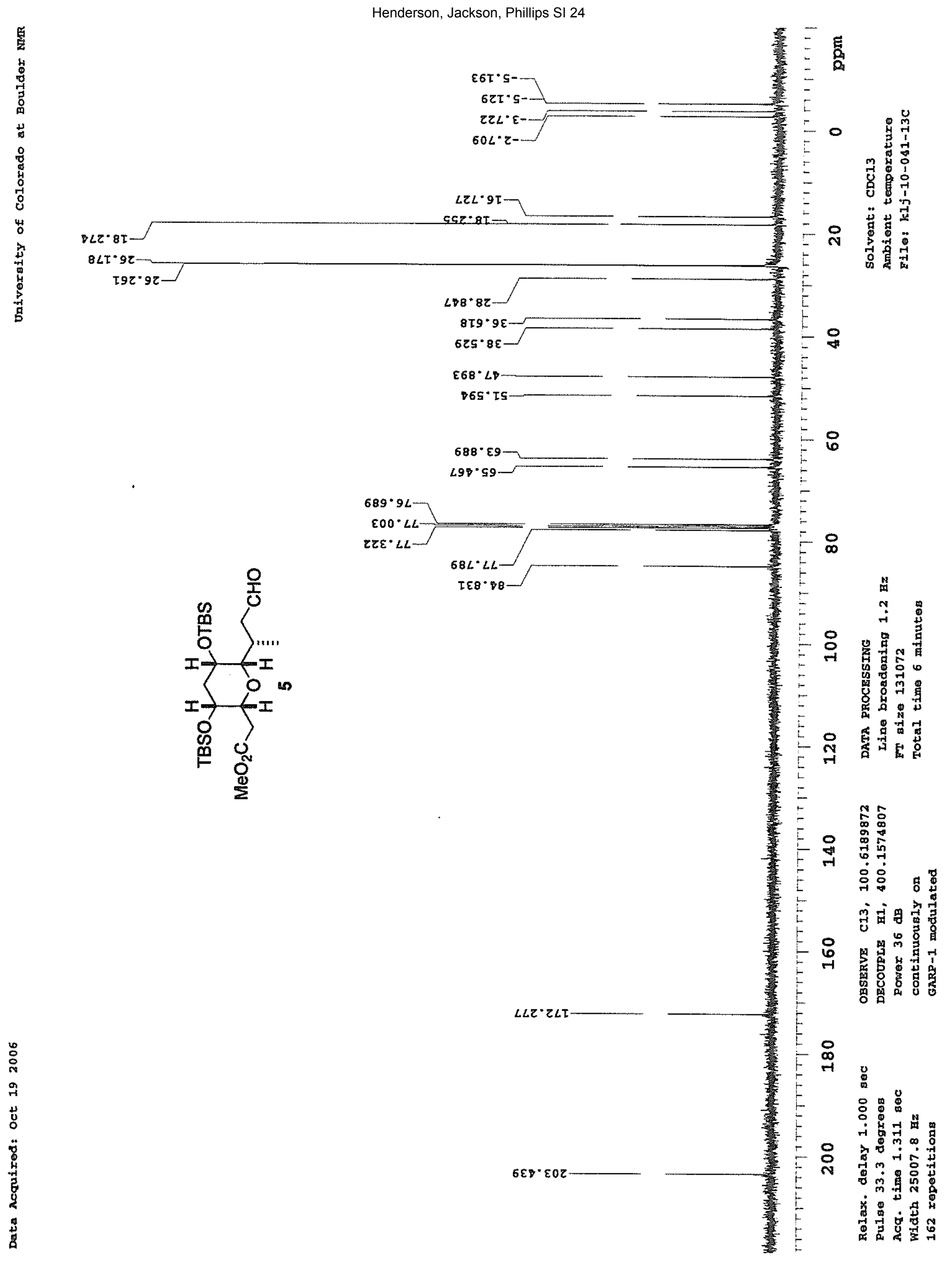


Henderson, Jackson, Phillips SI 25

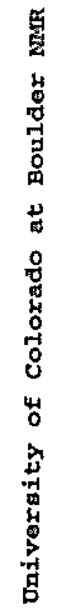
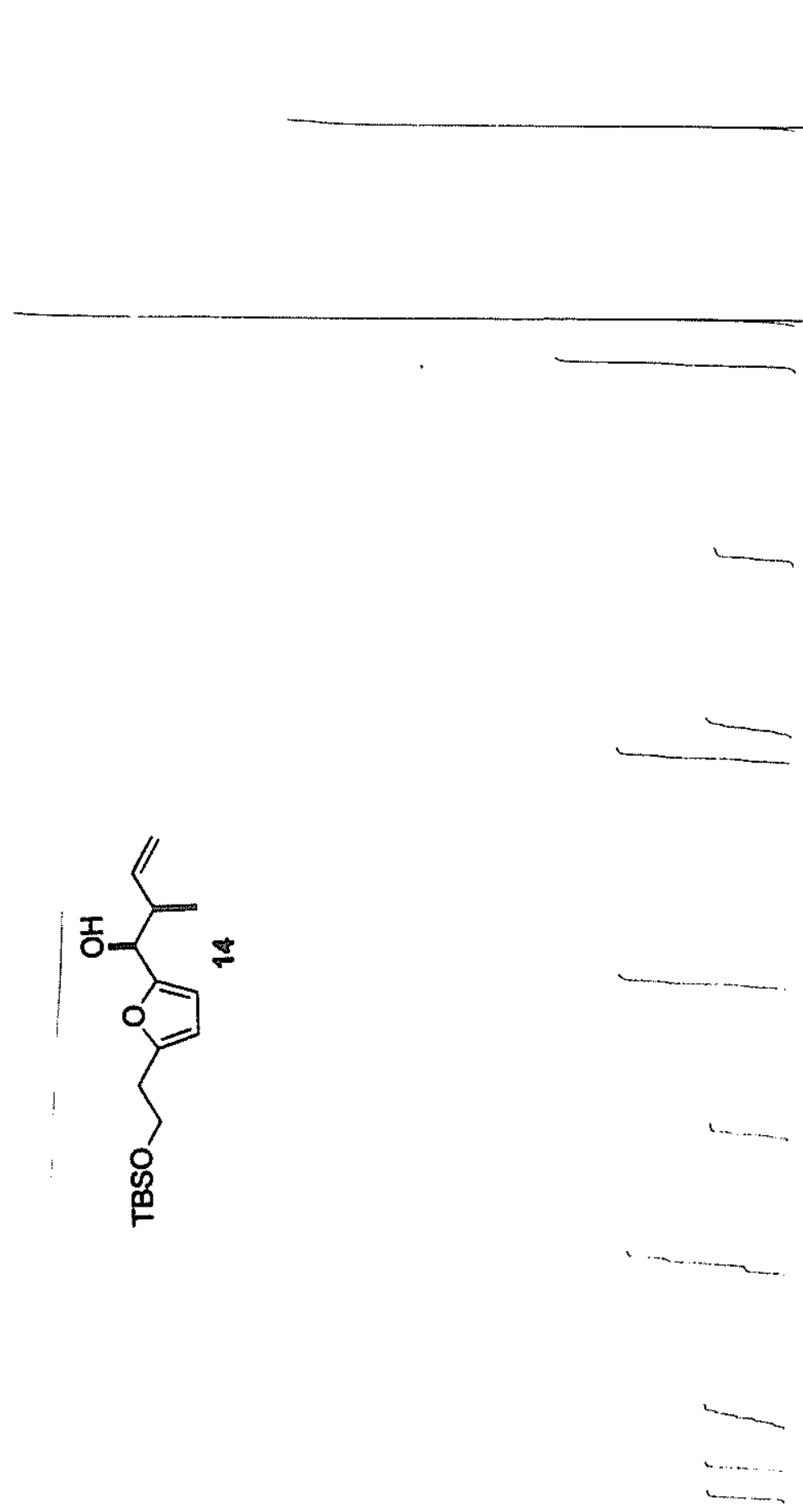
Henderson, Jackson, Phillips SI 26

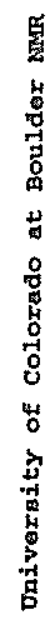

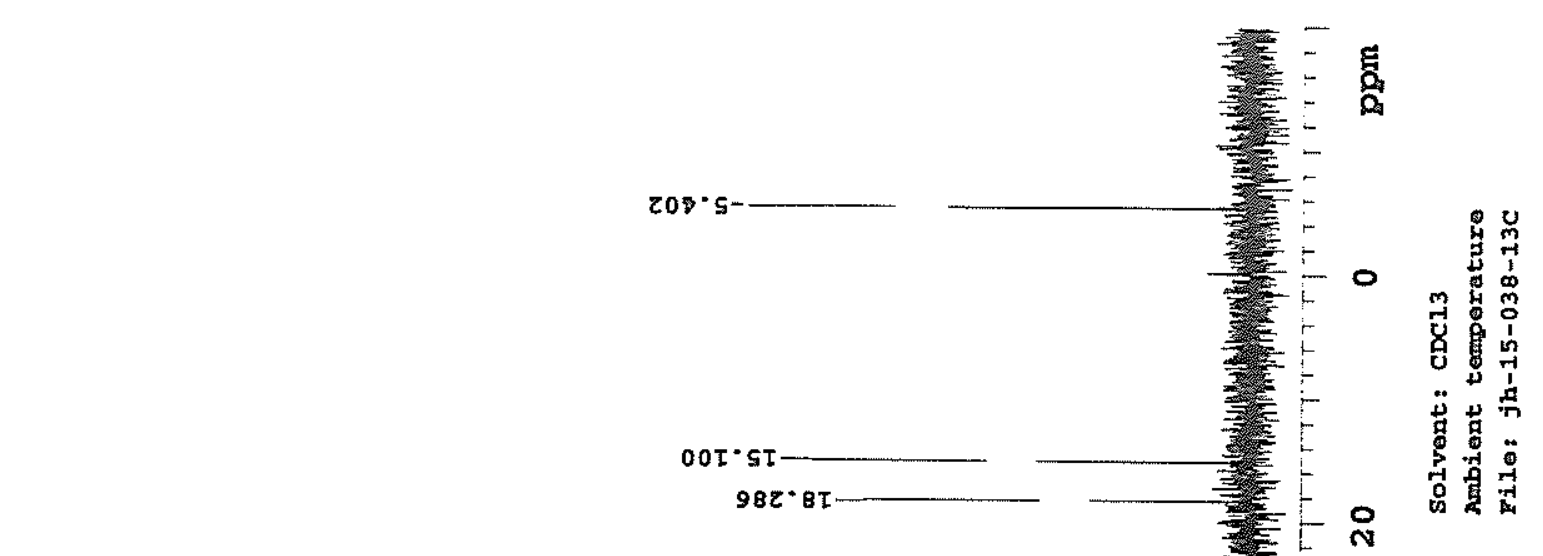

$269 \cdot 9 L$

$0 \angle B^{*} \mathrm{sz}$

$\angle 00^{\circ} \angle L$

$9 z \varepsilon^{\circ} L L$
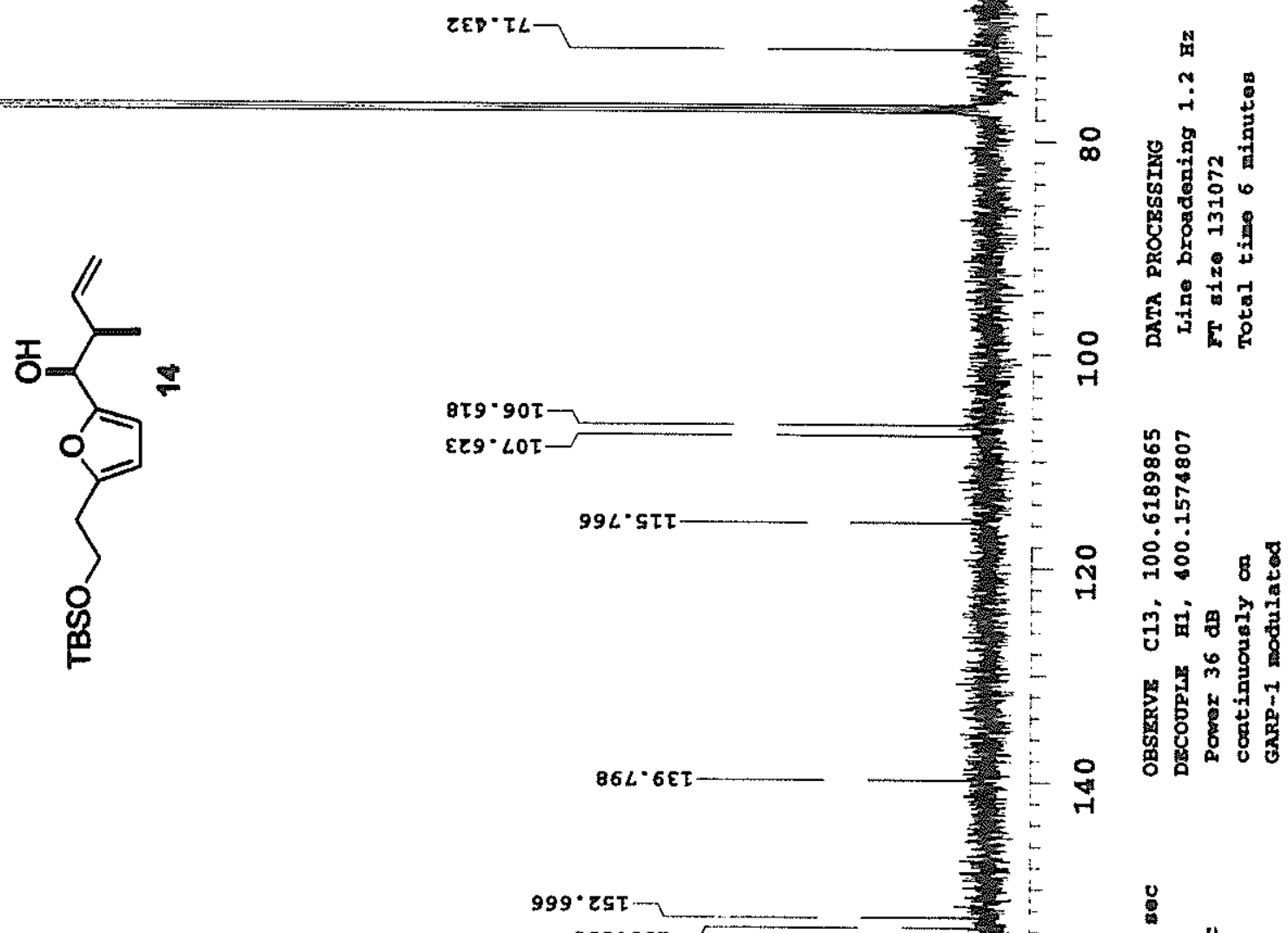

S6S. Est

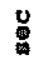

$\circ \quad$ : :

足

o

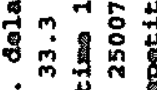


Henderson, Jackson, Phillips SI 27

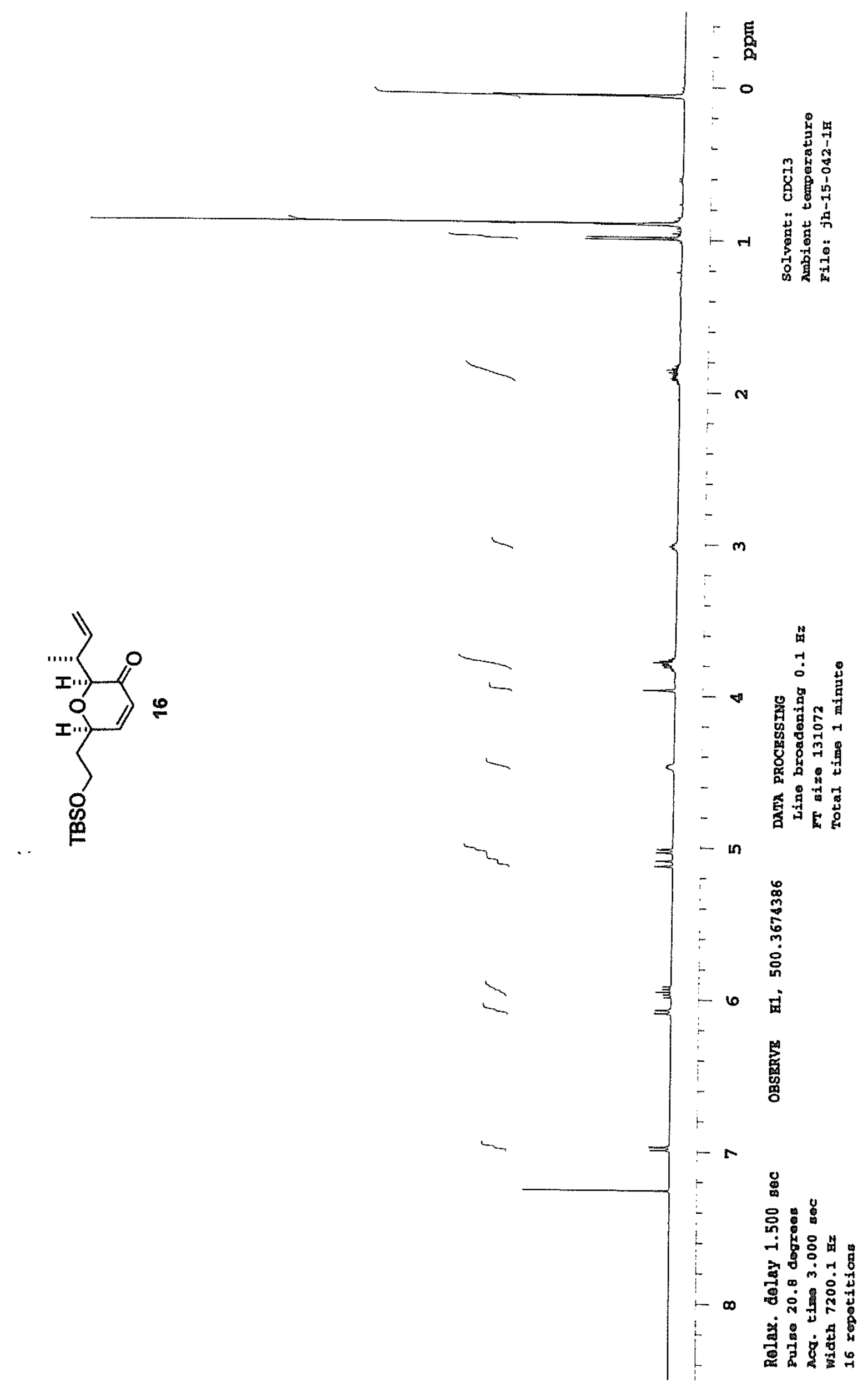


Henderson, Jackson, Phillips SI 28

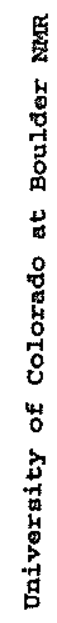

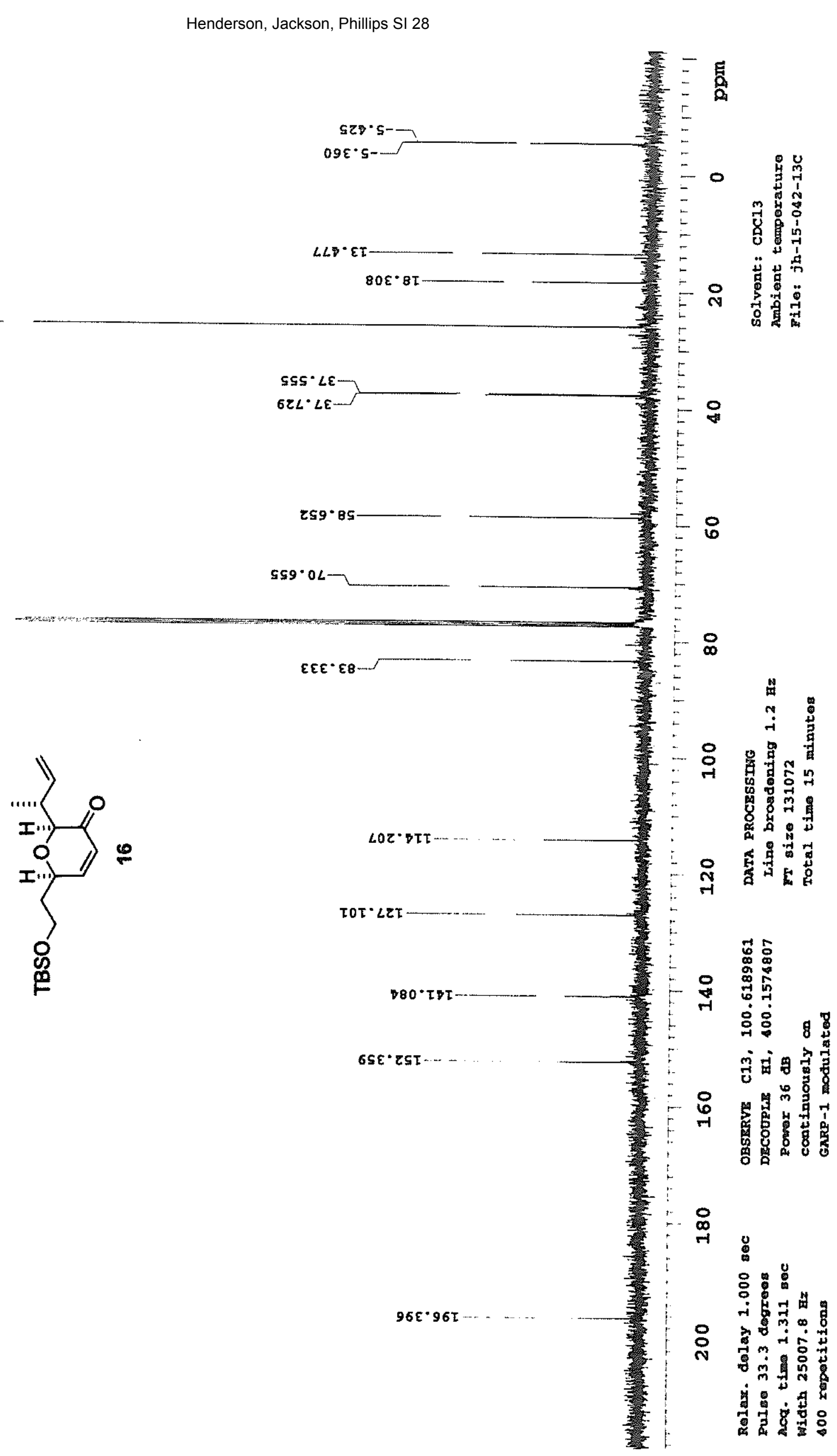


Henderson, Jackson, Phillips SI 29

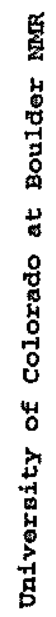

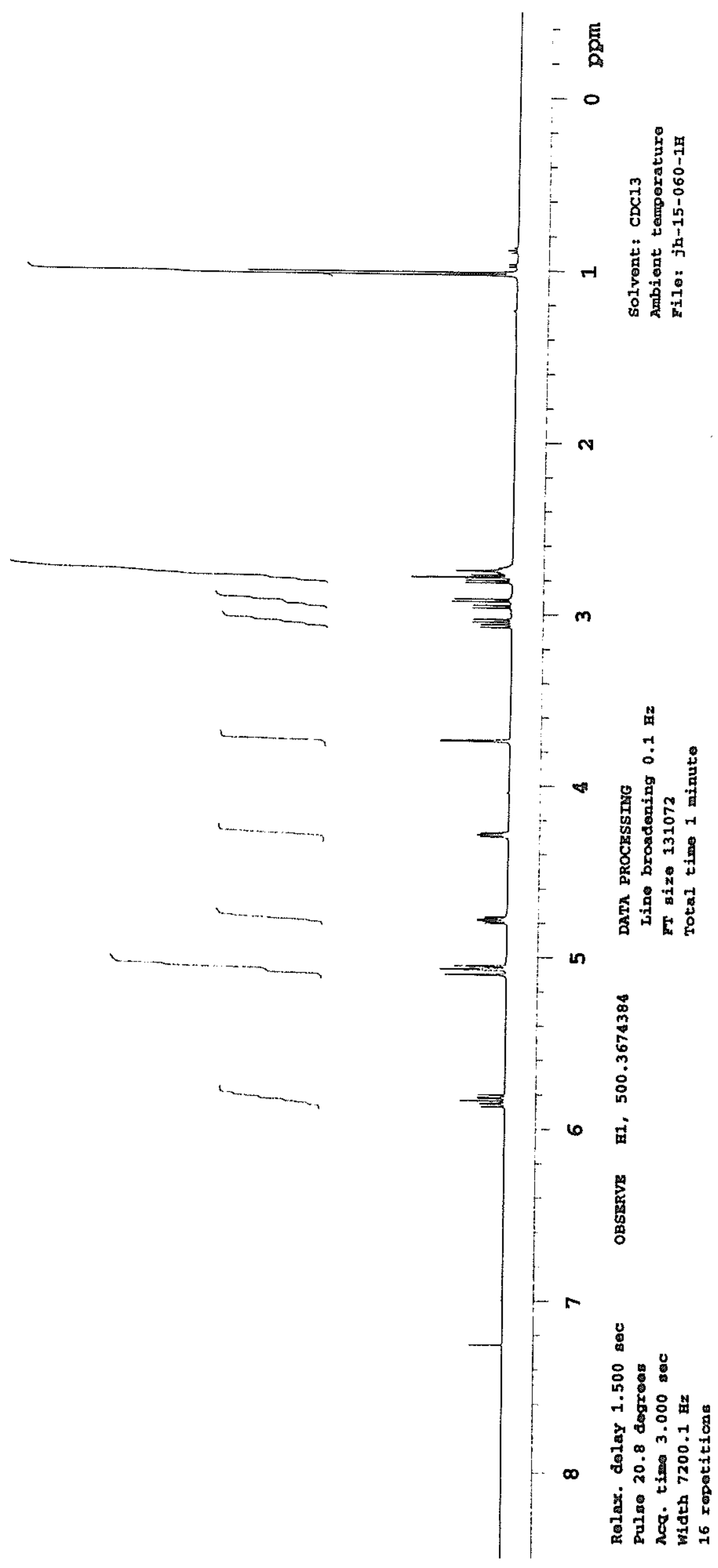


Henderson, Jackson, Phillips SI 30

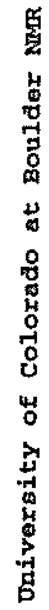

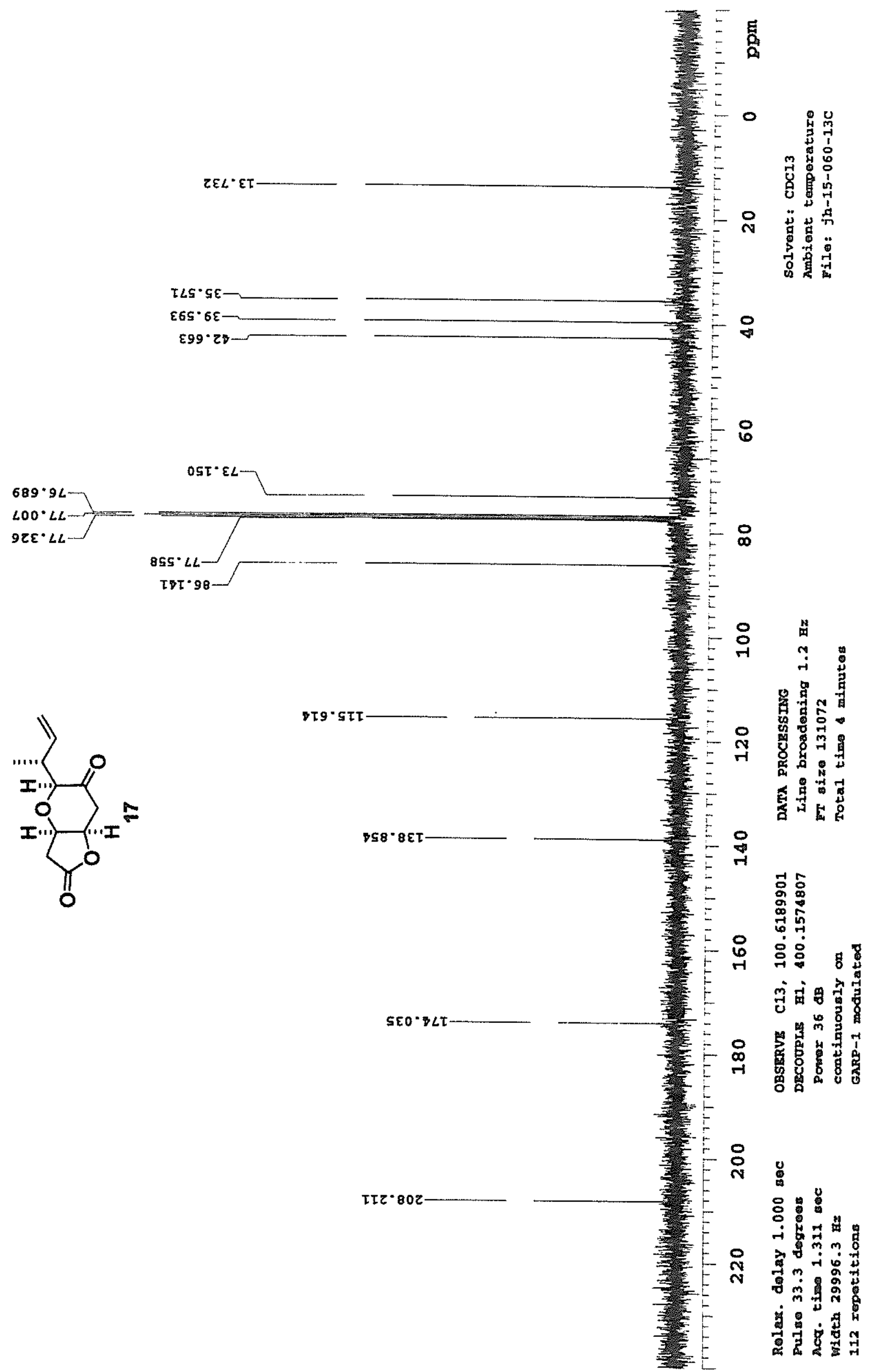


Henderson, Jackson, Phillips SI 31

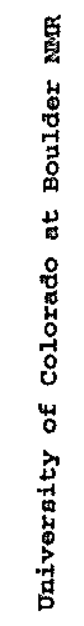

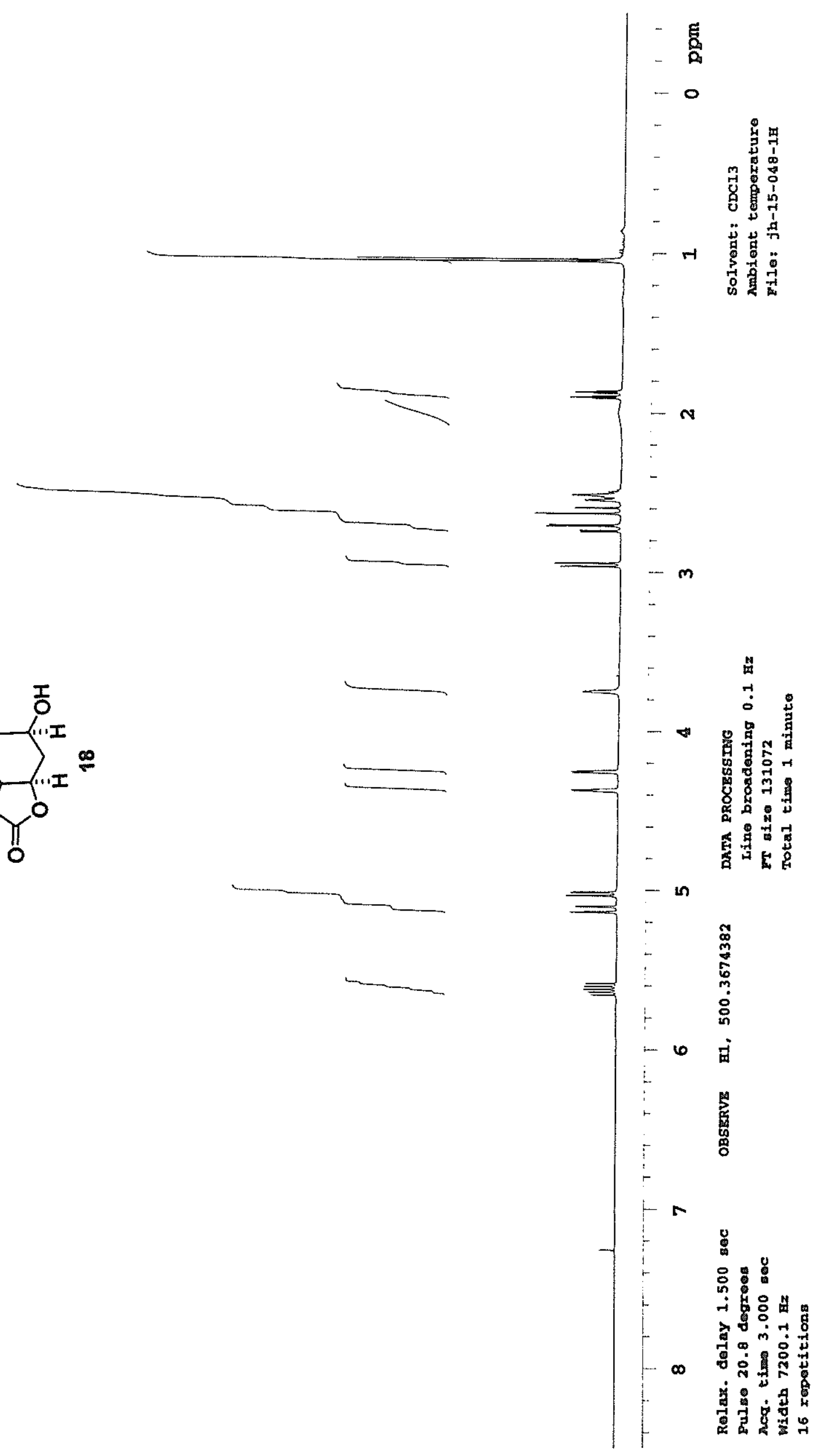




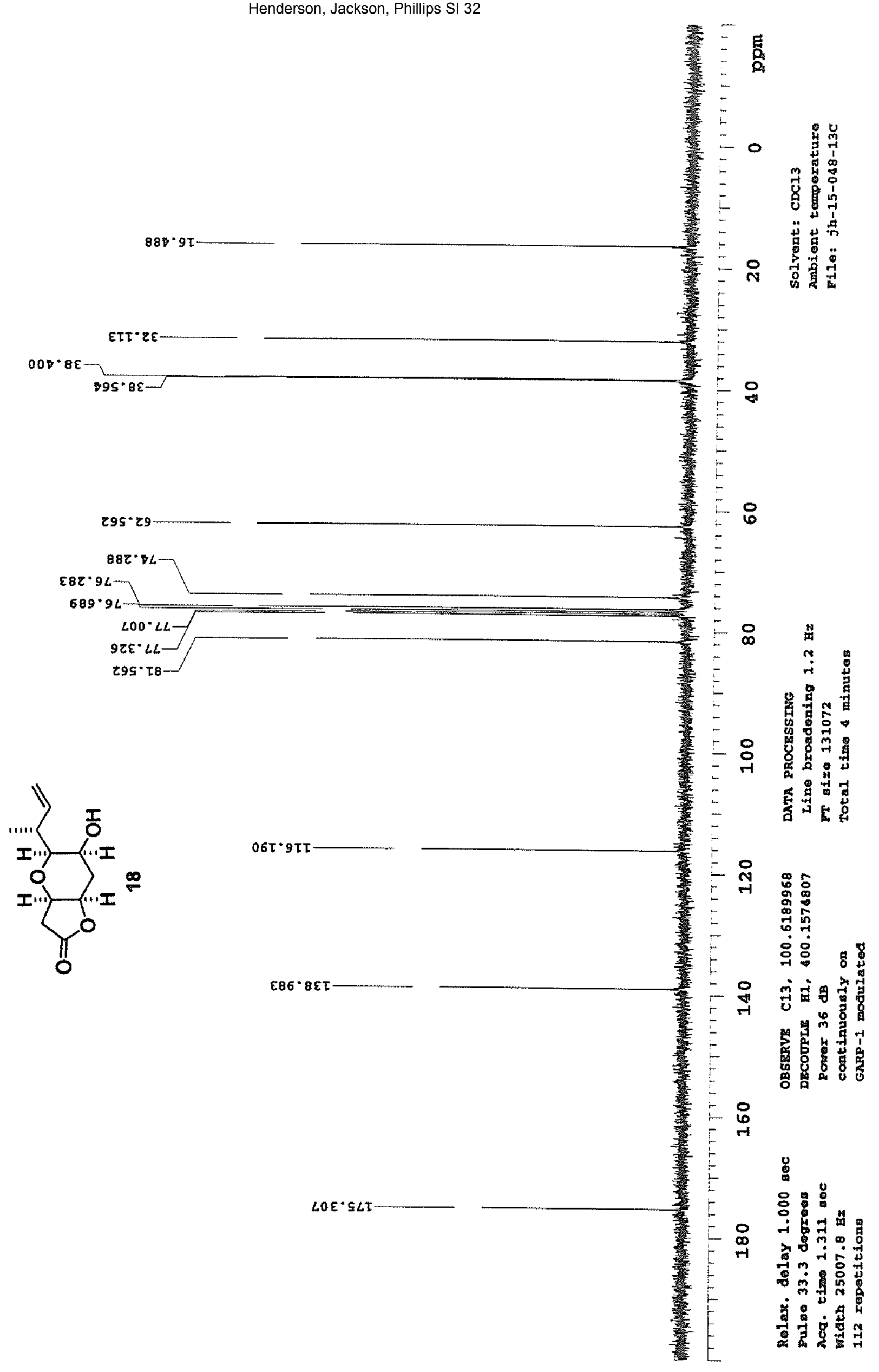


Henderson, Jackson, Phillips SI 33

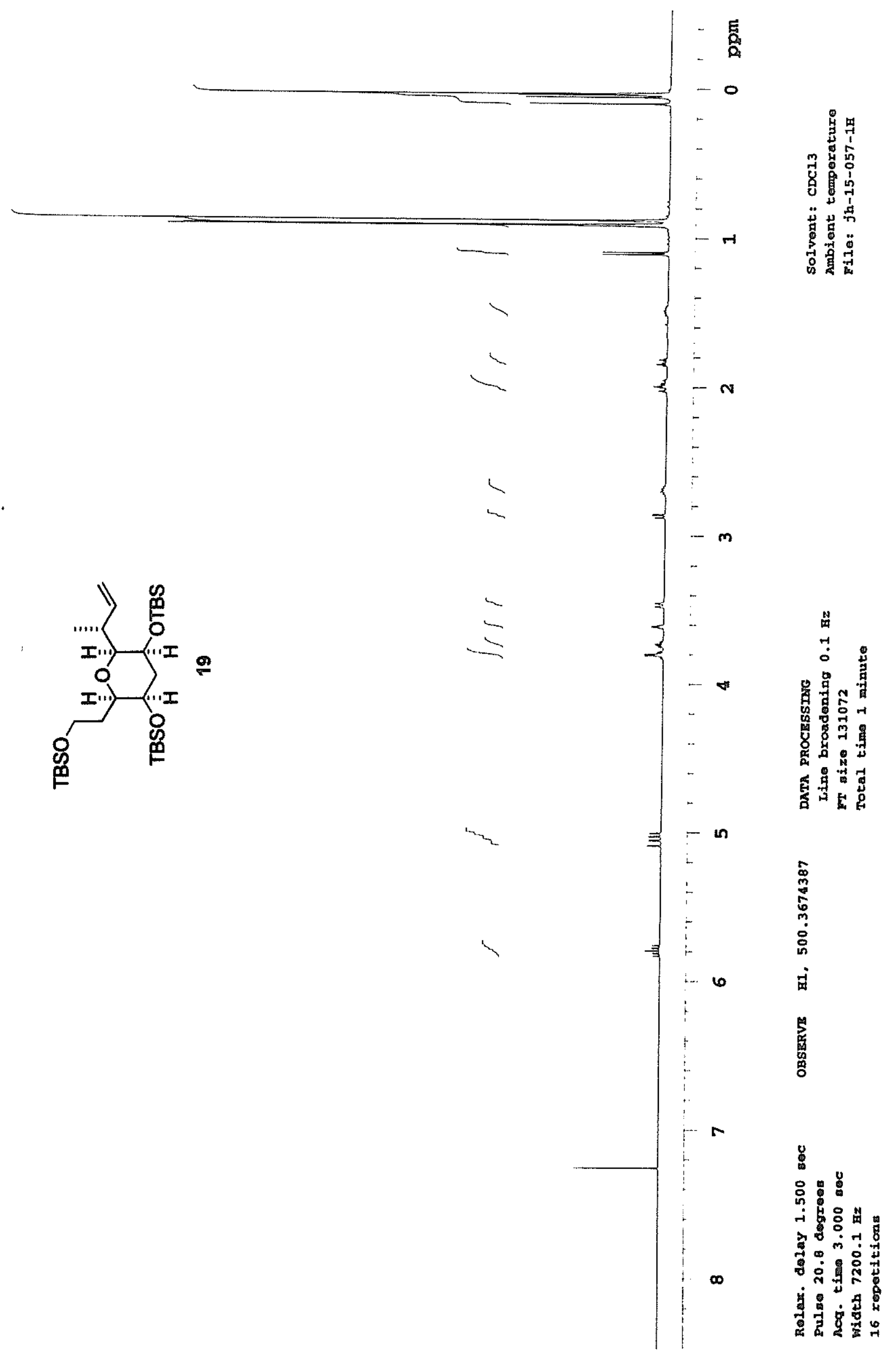


Henderson, Jackson, Phillips SI 34
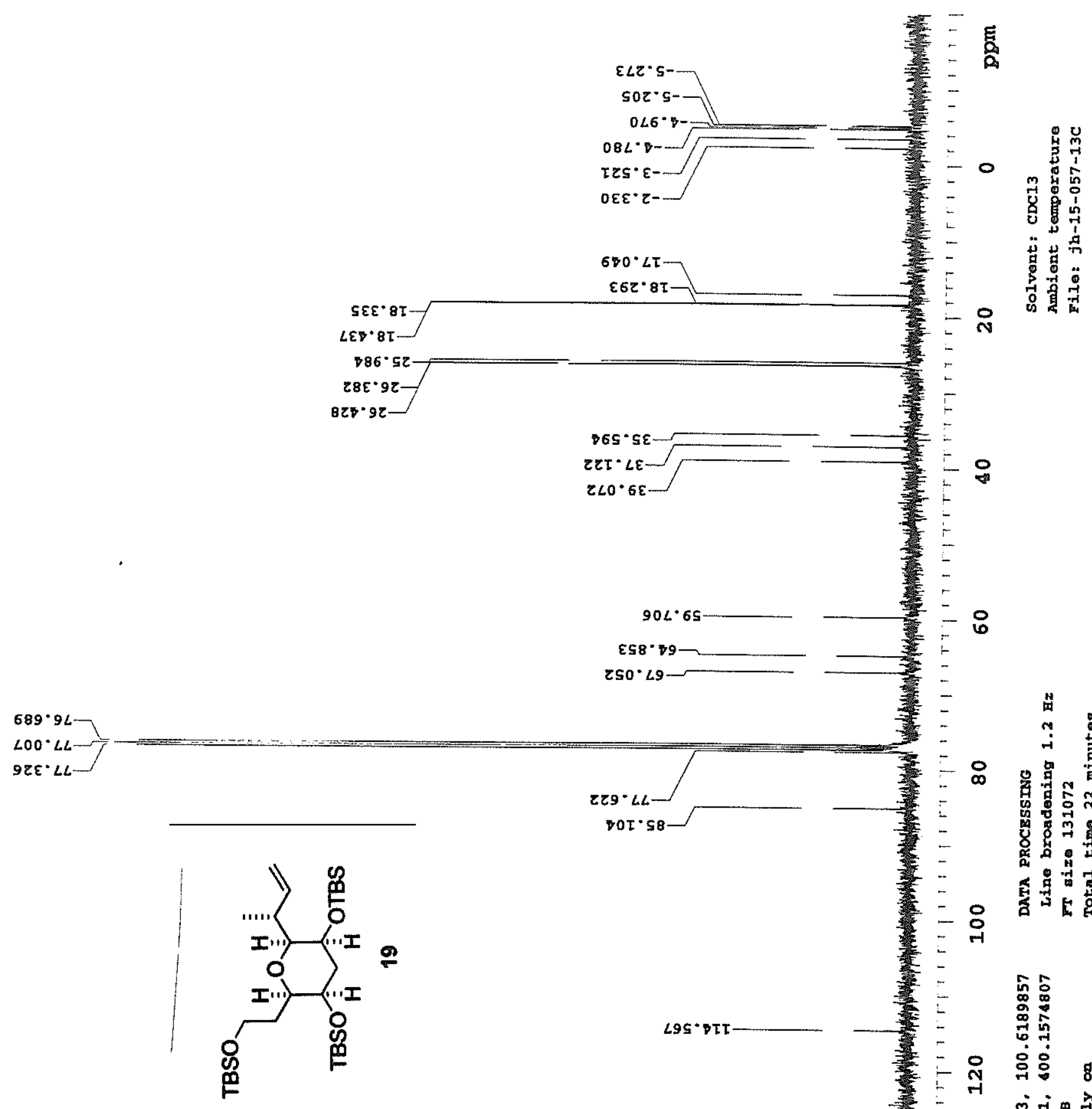
Henderson, Jackson, Phillips SI 35

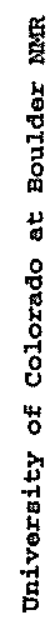

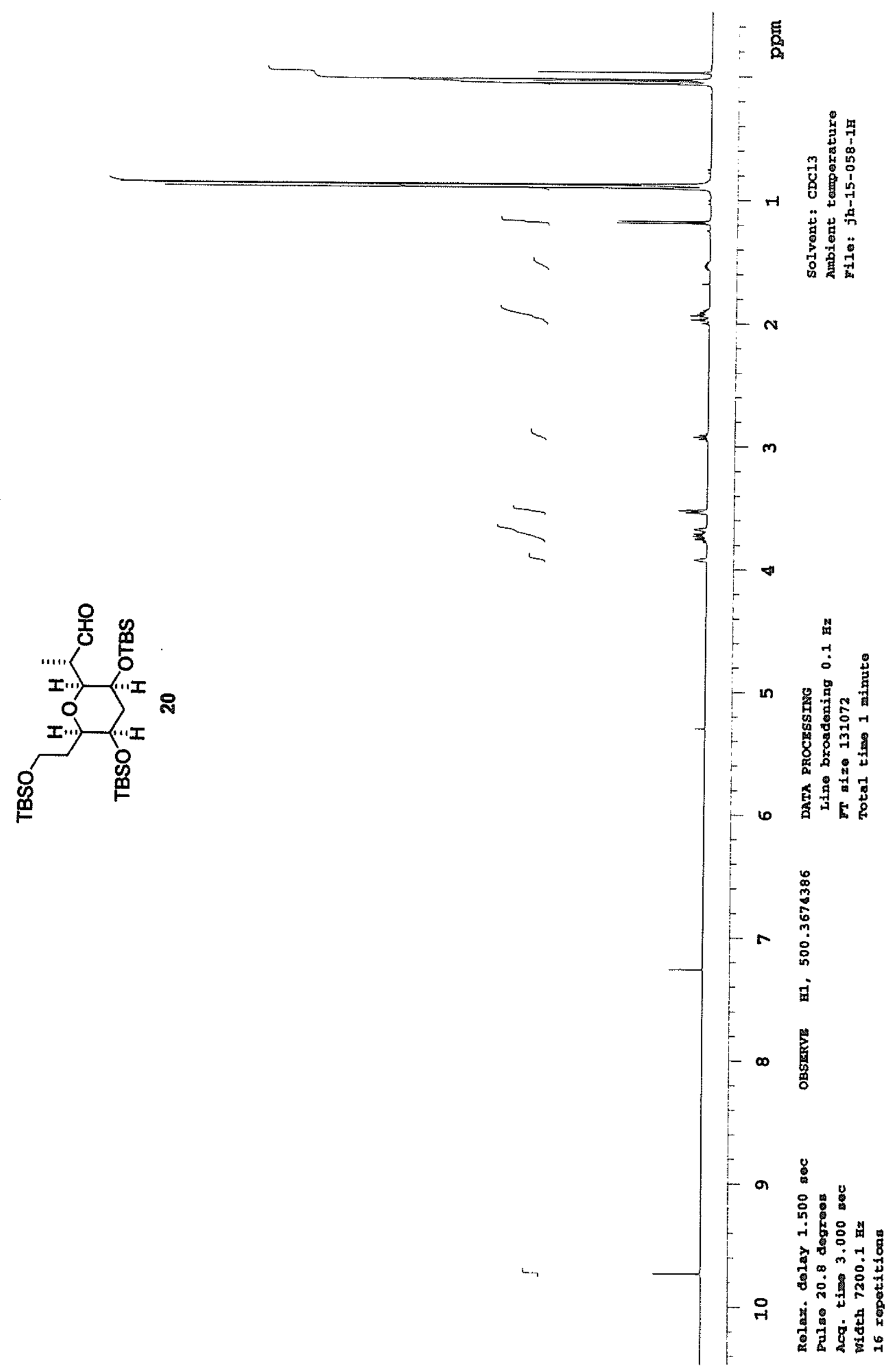


Henderson, Jackson, Phillips SI 36

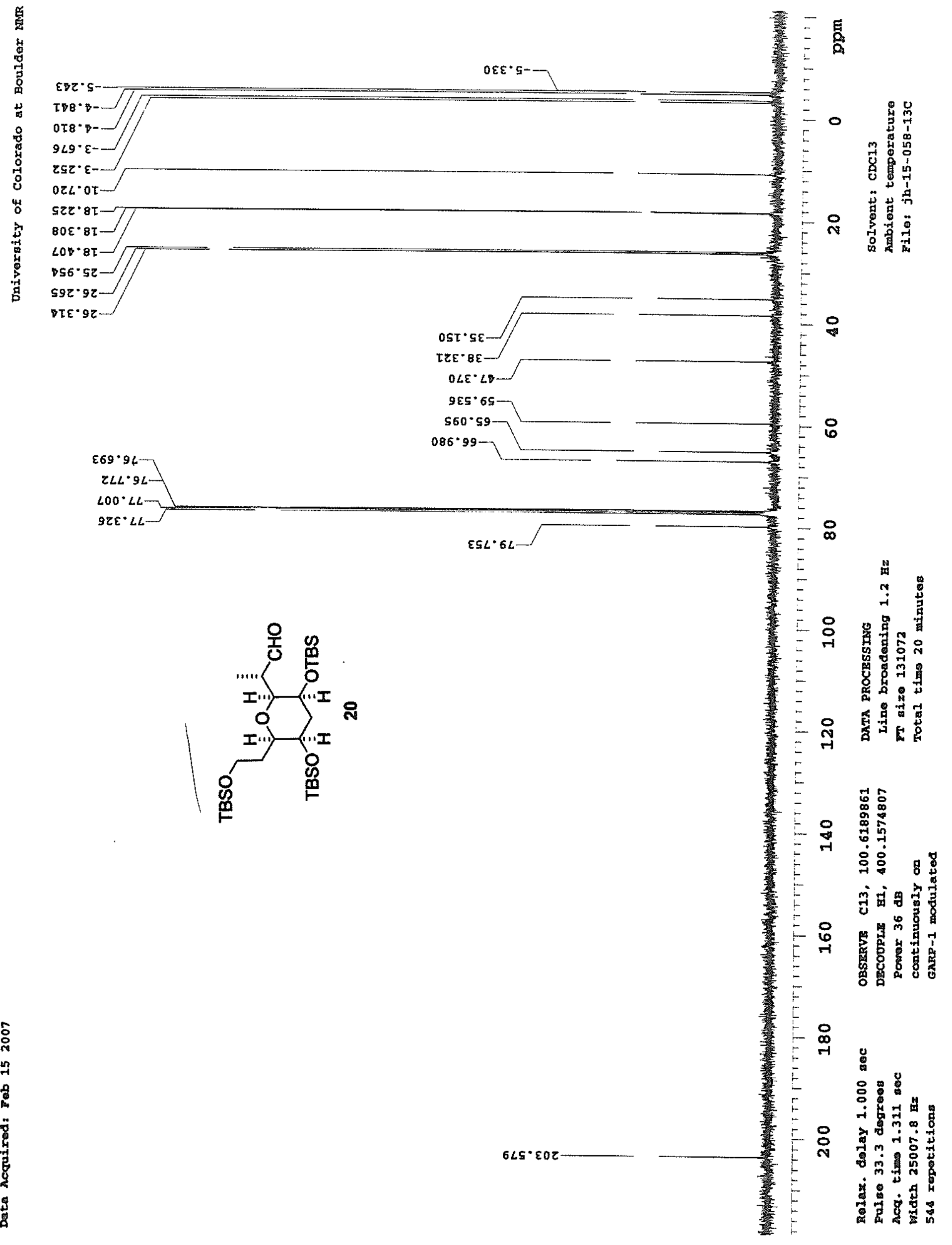


Henderson, Jackson, Phillips SI 37
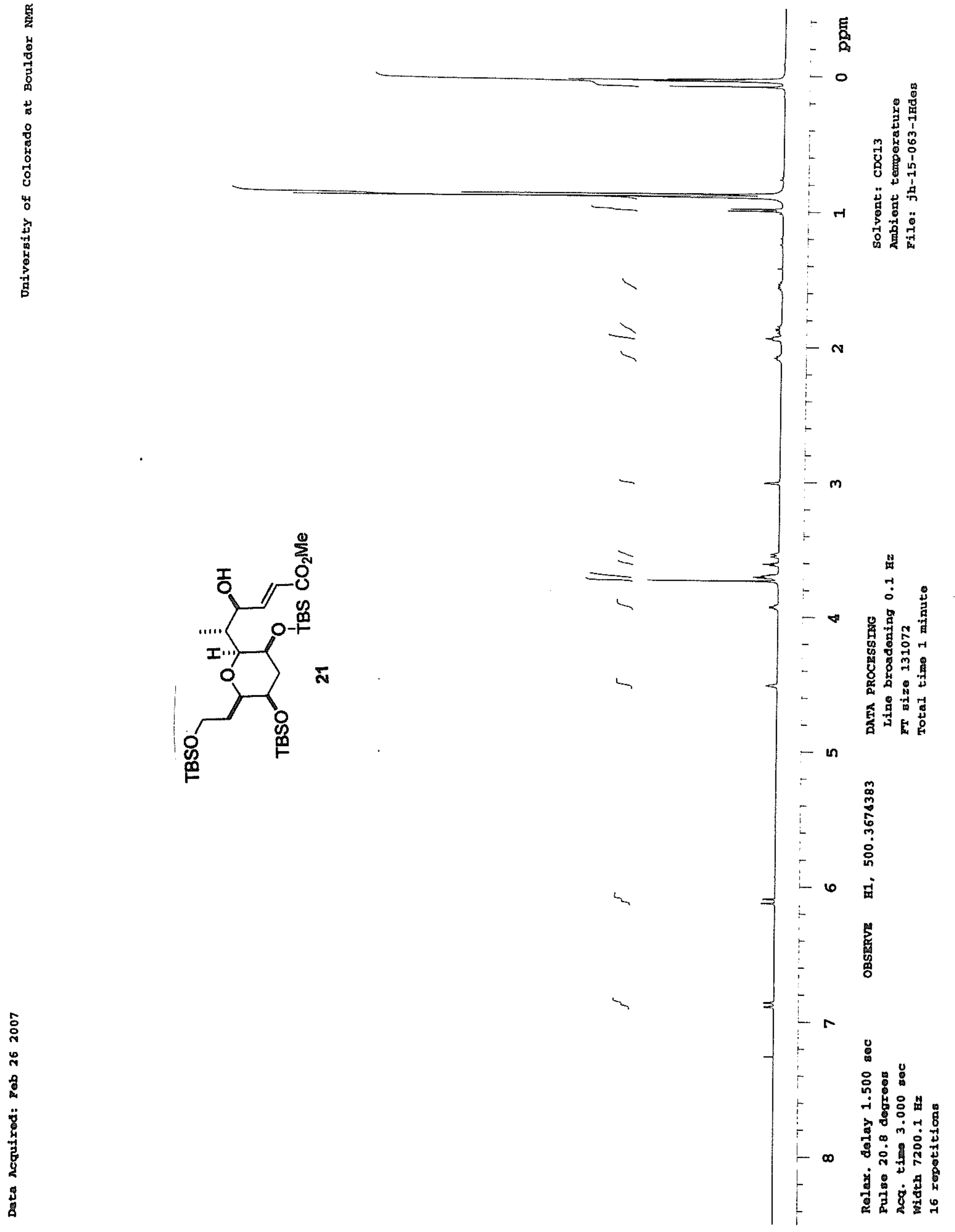
Henderson, Jackson, Phillips SI 38

要

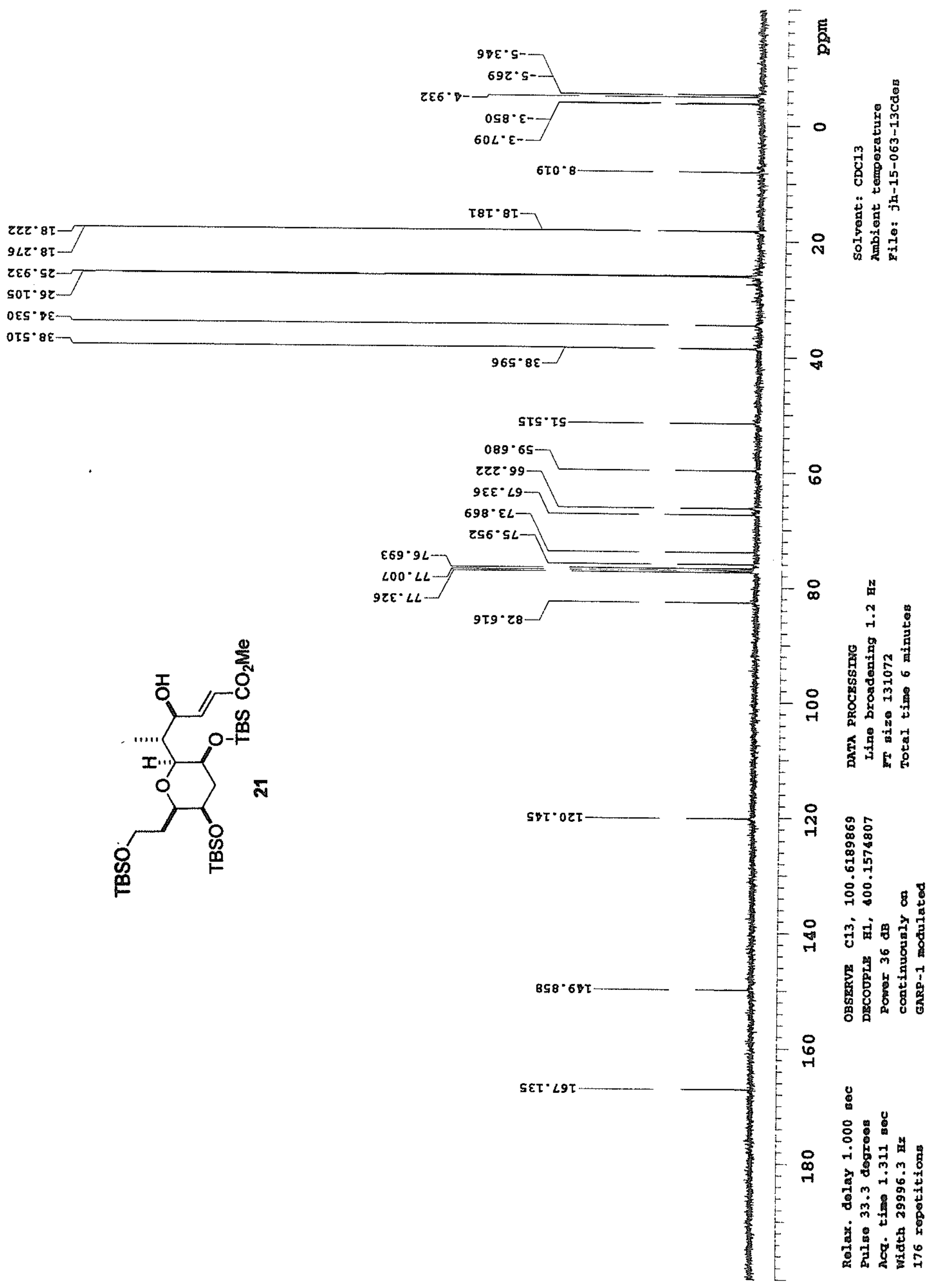


Henderson, Jackson, Phillips SI 39
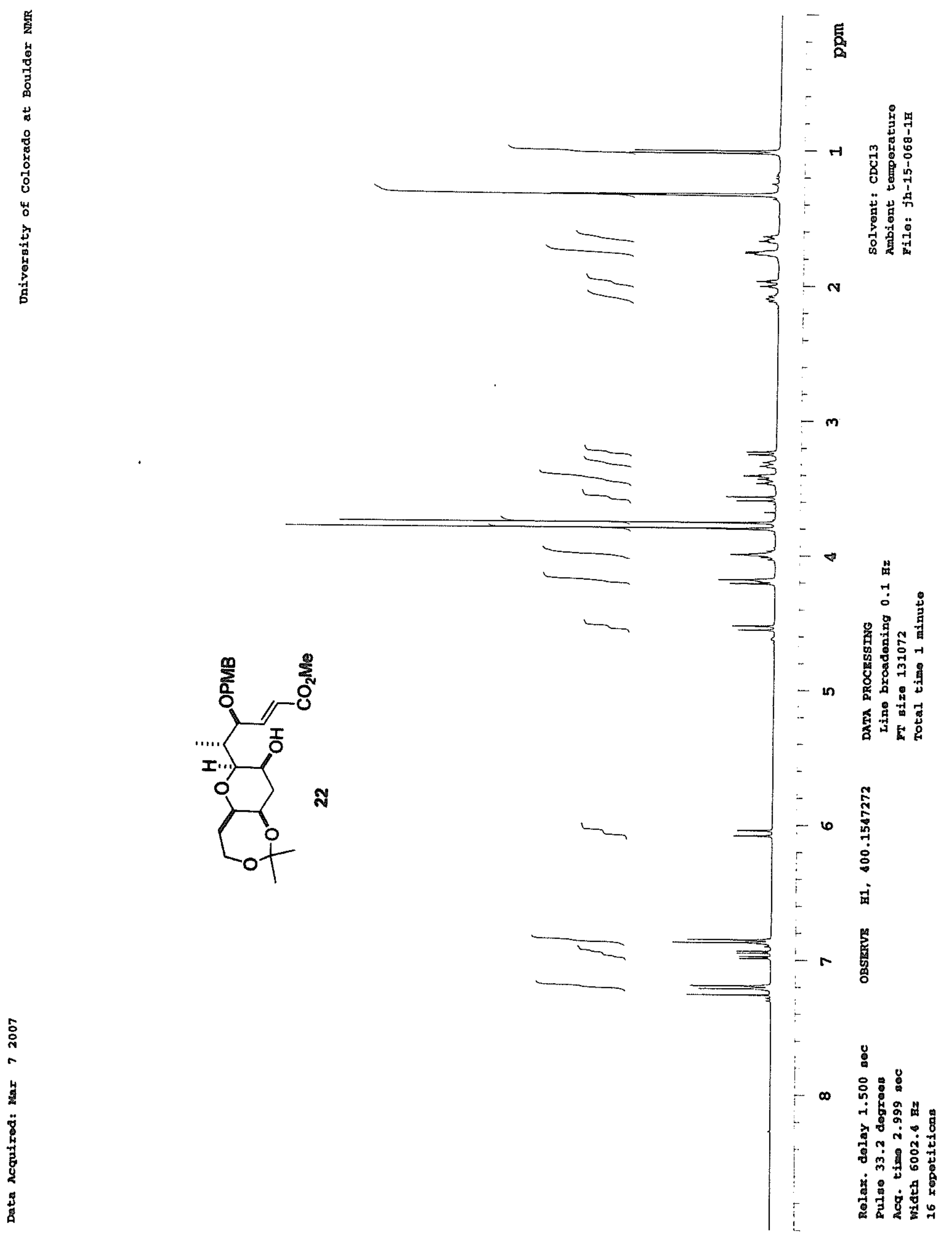
Henderson, Jackson, Phillips SI 40

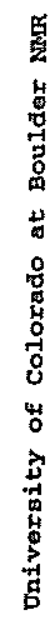

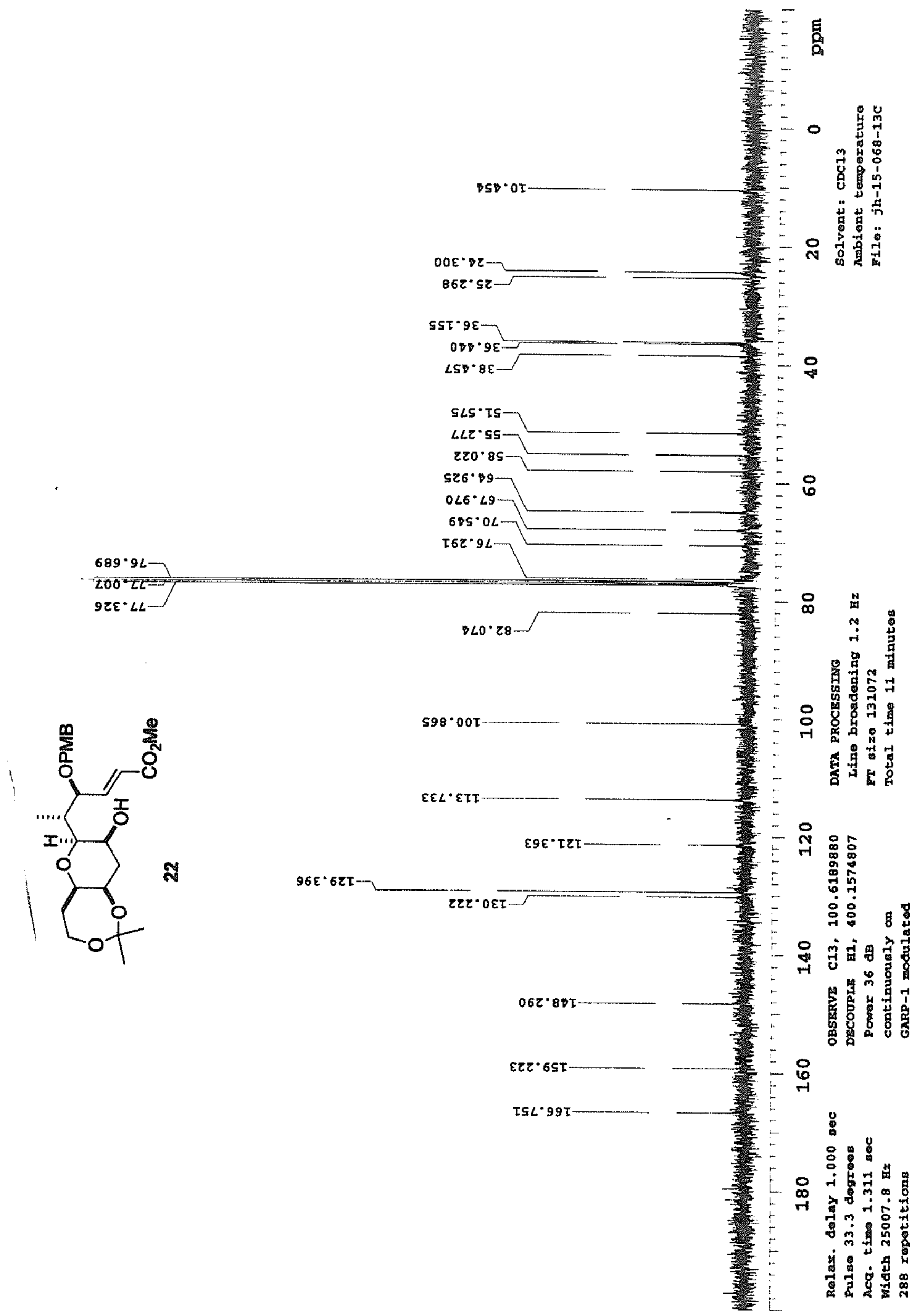


Henderson, Jackson, Phillips SI 41

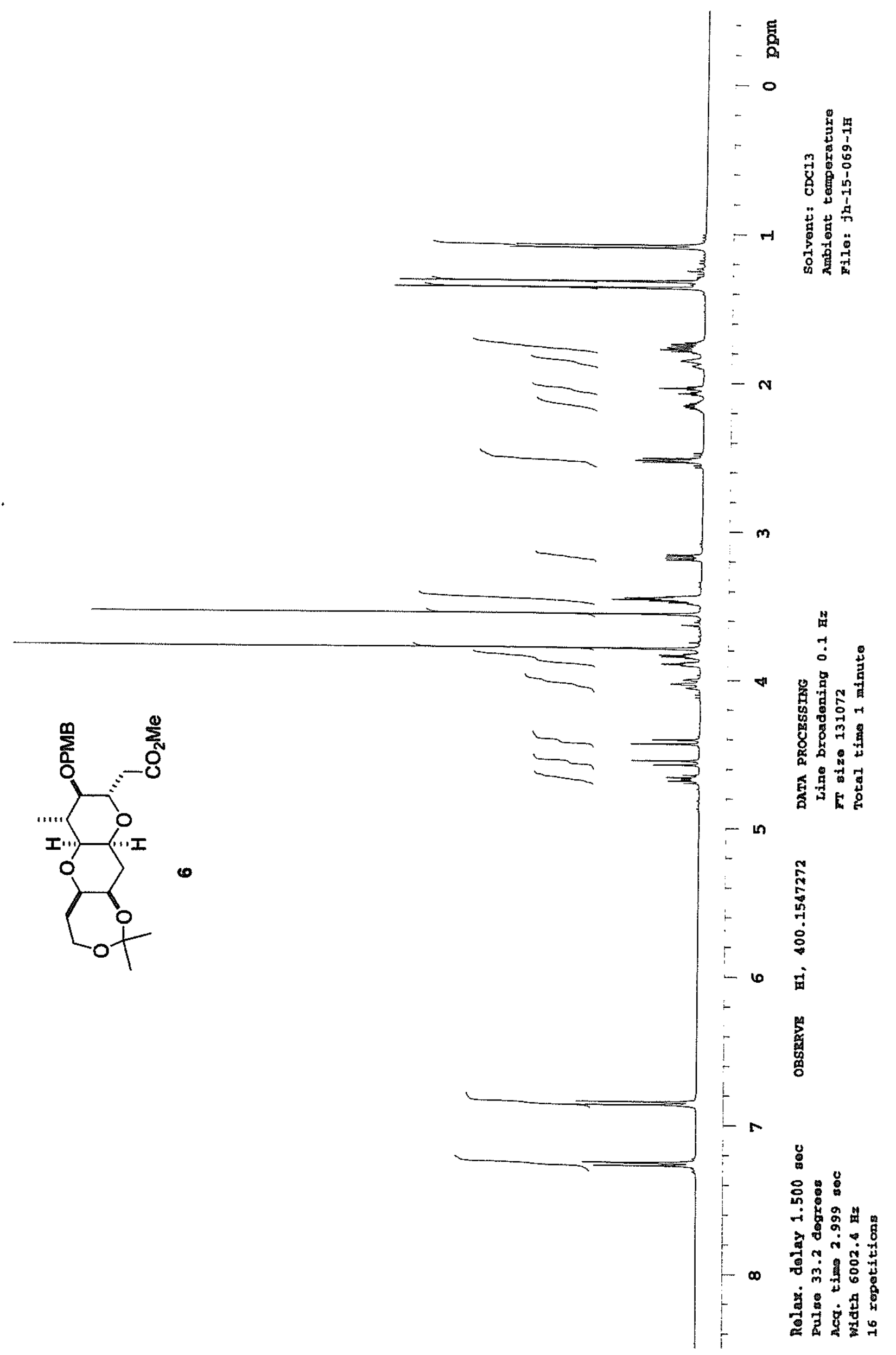


Henderson, Jackson, Phillips SI 42

\section{.}

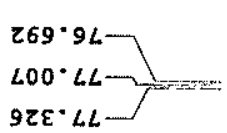<smiles>C[C@H]1O[C@H]2CCOC(C)(C)O[C@@H]2C[C@]1(C)F</smiles>

BLS·EII-

$88 \cdot 62 \tau$

$\bullet$
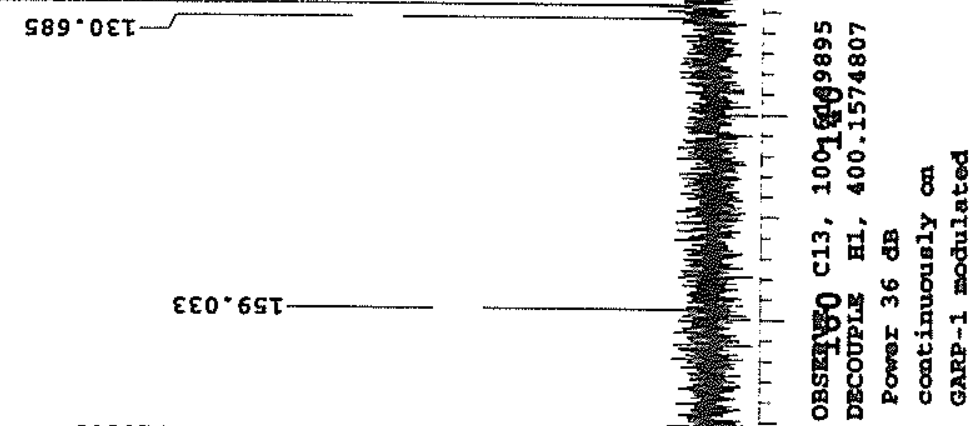